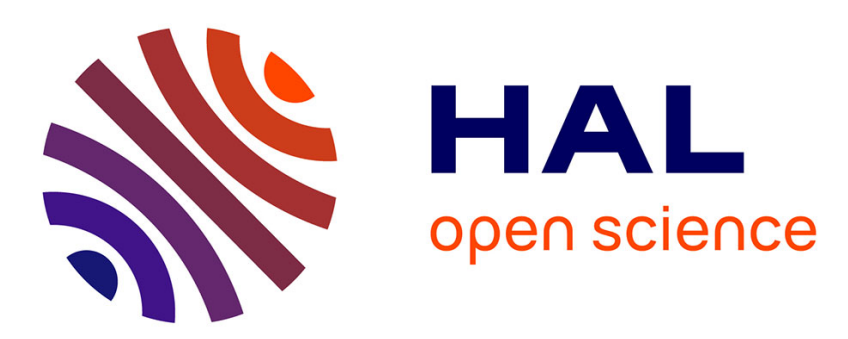

\title{
Near-field Radiative Heat Transfer in Many-Body Systems
}

\author{
S.-A Biehs, R Messina, P S Venkataram, A W Rodriguez, J C Cuevas, \\ Philippe Ben-Abdallah
}

\section{- To cite this version:}

S.-A Biehs, R Messina, P S Venkataram, A W Rodriguez, J C Cuevas, et al.. Near-field Radiative Heat Transfer in Many-Body Systems. Reviews of Modern Physics, 2021, 93 (2), 10.1103/RevModPhys.93.025009 . hal-03371841

\section{HAL Id: hal-03371841 https://hal.science/hal-03371841}

Submitted on 8 Oct 2021

HAL is a multi-disciplinary open access archive for the deposit and dissemination of scientific research documents, whether they are published or not. The documents may come from teaching and research institutions in France or abroad, or from public or private research centers.
L'archive ouverte pluridisciplinaire HAL, est destinée au dépôt et à la diffusion de documents scientifiques de niveau recherche, publiés ou non, émanant des établissements d'enseignement et de recherche français ou étrangers, des laboratoires publics ou privés. 


\title{
Near-field Radiative Heat Transfer in Many-Body Systems
}

\author{
S.-A. Biehs \\ Institut für Physik, Carl von Ossietzky Universität, D-26111 Oldenburg, Germany. \\ R. Messina \\ Laboratoire Charles Fabry, UMR 8501, Institut d'Optique, CNRS, Université Paris-Saclay, 2 Avenue Augustin Fresnel, \\ 91127 Palaiseau Cedex, France.
}

P. S. Venkataram and A. W. Rodriguez

Department of Electrical Engineering, Princeton University, Princeton, New Jersey 08544, USA.

J. C. Cuevas

Departamento de Física Teórica de la Materia Condensada and Condensed Matter Physics Center (IFIMAC), Universidad Autónoma de Madrid, E-28049 Madrid, Spain

P. Ben-Abdallah

Laboratoire Charles Fabry, UMR 8501, Institut d'Optique, CNRS, Université Paris-Saclay, 2 Avenue Augustin Fresnel, 91127 Palaiseau Cedex, France. and

Université de Sherbrooke, Department of Mechanical Engineering, Sherbrooke, PQ J1K 2R1, Canada ${ }^{*}$

\begin{abstract}
Many-body physics aims to understand emergent properties of systems made of many interacting objects. This article reviews recent progress on the topic of radiative heat transfer in many-body systems consisting of thermal emitters interacting in the near-field regime. Near-field radiative heat transfer is a rapidly emerging field of research in which the cooperative behavior of emitters gives rise to peculiar effects which can be exploited to control heat flow at the nanoscale. Using an extension of the standard Polder and van Hove stochastic formalism to deal with thermally generated fields in $N$-body systems, along with their mutual interactions through multiple scattering, a generalized Landauer-like theory is derived to describe heat exchange mediated by thermal photons in arbitrary reciprocal and non-reciprocal multi-terminal systems. In this review, we use this formalism to address both transport and dynamics in these systems from a unified perspective. Our discussion covers: (i) the description of non-additivity of heat flux and its related effects, including fundamental limits as well as the role of nanostructuring and material choice, (ii) the study of equilibrium states and multistable states, (iii) the relaxation dynamics (thermalization) toward local and global equilibria, (iv) the analysis of heat transport regimes in ordered and disordered systems comprised of a large number of objects, density and range of interactions, and (v) the description of thermomagnetic effects in magneto-optical systems and heat transport mechanisms in non-Hermitian many-body systems. We conclude this review by listing outstanding challenges and promising future research directions.
\end{abstract}

\section{Contents}

I. Introduction

II. Two-body systems

A. Parallel plates

B. Metals vs. dielectrics

C. Non-reciprocal materials

D. Nanostructuring and Roughness

1. Multilayer structures and photonic crystals

2. Metamaterials

3. Gratings and Metasurfaces

4. Graphene

5. Surface roughness

E. Impact of geometry

F. Numerical methods

1. Spectral methods

2. Decomposition methods

G. Upper bounds on near-field heat transfer

*Electronic address: pba@institutoptique.fr
III. Many-body systems

A. Heat flux in dipolar many-body systems

1. Light absorption in dipolar systems

2. Exchanged Power and Poynting vector

3. Non-additivity in many-dipole systems

4. T-DDA (as example of application)

B. Heat flux in macroscopic many-body systems

1. Scattering-matrix formalism

2. Non-additivity in many-body systems

3. Steady-state temperatures and multistable states

C. Radiative heat transfer in reciprocal many-body systems

1. Kinetic approach vs exact calculations

2. Heat transfer in complex networks 31

3. Long range heat transport and amplification of heat flux

4. Relaxation dynamics

5. Dynamical control

6. Heat transport regimes

D. Non-reciprocal systems

1. General discussion

2. General impact of non-reciprocity

3. Magneto-optical nano-particles

4. Giant magneto-resistance 
5. Persistent heat flux, angular momentum, spin and heat current

6. Hall effect for thermal radiation

7. Heat flux rectification with non-reciprocal surface waves

\section{Outlook and open questions}

Acknowledgments

\section{3}

44

46

77

References

\section{INTRODUCTION}

Heat transfer in a given system is in its simplest sense (i.e. ignoring multiple irreversible transport processes (Onsager, 1931)) thermal energy in transit due to a spatial temperature difference (Bergman et al., 2011). There are three basic heat transfer modes: conduction, convection, and radiation. In the case of a stationary medium, which could be a solid or a fluid, conduction refers to heat transfer through local agitation of atoms or charges that occurs across the medium in response to a temperature gradient. Ultimately, the carriers responsible for heat conduction are phonons, molecular vibrations or electrons/ions in the case of electrical conductors. The second mode of transport is convection, and refers to heat transfer that occurs between a surface and a moving fluid when they are at different temperatures (or by advection inside the fluid itself). Finally, the third heat transfer mechanism is thermal radiation, which is the topic of this review. All bodies at a finite temperature emit energy in the form of electromagnetic waves (or photons). Hence, even in the absence of an intervening medium, there is always heat transfer via thermal radiation between bodies at different temperatures. This makes thermal radiation one of the most ubiquitous physical phenomena and its understanding of critical importance for many different areas of science and engineering (Howell et al. 2016 , Modest, 2013, Zhang, 2007).

Traditionally, our understanding of thermal radiation is based on Planck's law (Planck, 1914), which establishes that a black body (an object that absorbs all the radiation that impinges on it) emits thermal radiation following a broadband distribution that only depends on the body's temperature. Planck's law provides a unified description of a variety of thermal radiation phenomena and, in particular, it sets an upper limit (StefanBoltzmann's law) for the radiative heat transfer (RHT) between bodies. However, Planck's law was derived using ray optics and hence, it is expected to fail when the spatial dimensions in a thermal problem are smaller than or comparable to the thermal wavelength $\lambda_{\mathrm{Th}}$ defined by Wien's displacement law $(\sim 10 \mu \mathrm{m}$ at room temperature) (Planck, 1914). In particular, Planck's law fails to describe RHT between objects separated by distances $\lesssim \lambda_{\text {Th }}$ (Pendry, 1999, Volokitin and Persson, 2007). In this near-field regime, RHT can be dominated by evanescent waves (or photon tunneling), not taken into account in Planck's law, and the Planckian (or black-body) limit can be greatly overcome by bringing objects sufficiently close, see Fig. 1. This phenomenon was first predicted within the rigorous framework of fluctuational electrodynamics (FE) (Rytov et al. 1989) by Polder and Van Hove in the early 1970s (Polder and Hove, 1971), see Sec. II. This near-field radiative heat transfer (NFRHT) enhancement was first hinted in several experiments in the late 1960s (Domoto et al., 1970, Hargreaves, 1969), but it was not firmly confirmed until the 2000s (Hu et al., 2008; Kittel et al., 2005, Narayanaswamy et al., 2008; Rousseau et al. 2009 Shen et al. 2009). Since then, numerous experiments exploring different aspects of NFRHT have been reported and they have boosted the field of thermal radiation (Bernardi et al., 2016, DeSutter et al., 2019, Fiorino et al., 2018a b c; Ghashami et al., 2018; Guha et al. | 2012, Ito et al., 2015, 2017; Kim et al. 2015 Králík et al. 2012, 2017; Lang et al., 2017, Lim et al. 2015; Musilová et al., 2019, Ottens et al., 2011. Shen et al. | 2012; Shi et al., 2013 ; Song et al., |2015b, 2016; St-Gelais et al., 2014, 2016, Worbes et al., 2013; van Zwol et al., 2012a b). These experiments have, in turn, generated hope that NFRHT may have an impact on different technologies such as heat-assisted magnetic recording, thermal lithography, scanning thermal microscopy, coherent thermal sources, near-field based thermal management, thermophotovoltaics, and other energy conversion devices, see (Basu et al., 2009, Cuevas and GarcíaVidal, 2018, Komiyama, 2019, Song et al., 2015a) and references therein.

In parallel to these experimental advances, over the last two decades, there has been a huge amount of theoretical activity. Initially, attention was devoted to the importance of choice of materials and the elucidation of the different mechanisms of near-field thermal radiation. In that regard, polar dielectrics exhibiting polaritonic resonances that lead to surface modes have played a prominent role in this field (Mulet et al. 2002). Then, following nanophotonics concepts, a lot of work has been devoted to assess the possibility of further enhancing NFRHT and to tune its spectral properties by using nanostructures such as thin films and multilayer systems (Ben-Abdallah et al. 2009a; Biehs, 2007; Biehs et al., 2007; Francoeur et al., 2008 , Maslovski et al., 2013, Volokitin and Persson, 2007), photonic crystals and gratings (Ben-Abdallah et al., 2010, Biehs et al., 2011; Guérout et al., 2012; Messina et al., 2017b; Rodriguez et al., 2011), and metasurfaces (Dai et al., 2016a) Fernández-Hurtado et al. 2017: Liu and Zhang, 2015a). The investigation of the use of metamaterials for further enhancing NFRHT (Biehs et al., 2011, 2012, Guo et al., 2012, Joulain et al., 2010) or low-dimensional materials like graphene or phosphorene to tune NFRHT (Ilic et al., 2012a; Liu et al., 2019, 2014a: Rodriguez-Lopez et al., 2015 Svetovoy et al. 2012; Volokitin and Persson, 2011; Zhang et al., 2018) has also attracted significant attention. Another topic of great importance has been the study of the active control of NFRHT by different means, including the use of phase- 


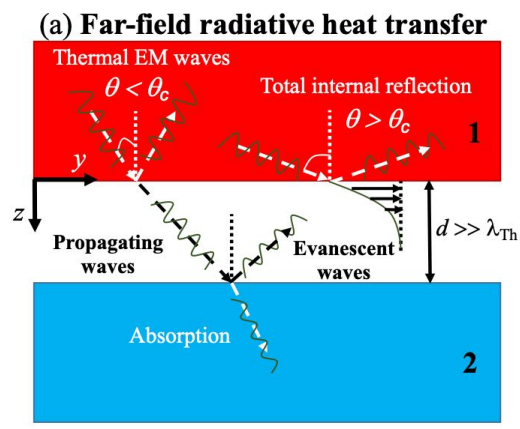

(b) Near-field radiative heat transfer

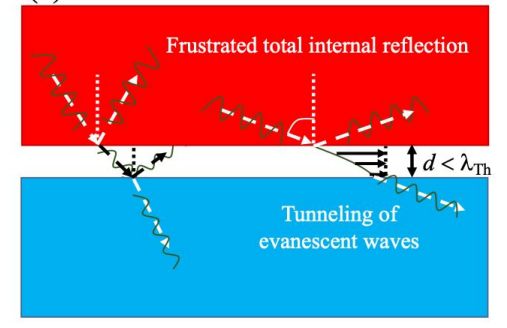

FIG. 1 (a) Far-field radiative heat transfer between two infinite parallel plates (media 1 and 2) separated by a vacuum gap. In this scenario, the gap size $d$ is much larger than the thermal wavelength, $\lambda_{\mathrm{Th}}$, and the two plates exchange heat only via propagating waves. The evanescent waves generated in the vacuum gap by total internal reflection are not able to reach the second plate and do not contribute to the heat transfer. (b) When $d<\lambda_{\text {Th }}$ the tunneling of evanescent waves can give a significant contribution to the radiative heat transfer and in this way the Planckian (or black-body) limit can be greatly overcome in this near-field regime.

transition materials (Menges et al., 2016, van Zwol et al. 2011a b), the application of an external magnetic field (Moncada-Villa et al. 2015), or the regulation of chemical potentials for photons with an external bias (Chen et al. 2015). There are also several theoretical proposals for functional devices that make use of NFRHT for thermal management (Ben-Abdallah and Biehs, 2015, Otey et al., 2010), thermophotovoltaics (Basu et al., 2007; Laroche et al. 2006; Narayanaswamy and Chen, 2003, Zhao et al. 2017a), and other energy applications (Chen et al. 2016, 2015). On a more fundamental level, quantum approaches based on the Huttner-Barnett model, quantum Langevin equations, non-equilibrium Green's function method, and the master-equation approach for open quantum systems have been proposed (Barton, 2016 Biehs and Agarwal, 2013a, Janowicz et al. 2003 , Sääskilahti et al., 2014; Sasihithlu and Agarwal 2018 , Wang and Peng, 2017).

From a broader perspective, a new general picture of RHT has emerged in recent years with profound similarities to other heat and charge transport phenomena, including phonon conduction in nanoscale systems and coherent electronic transport in mesoscopic devices (Cuevas and Scheer, 2017). In particular, RHT is now routinely described in terms of the Landauer formula, originally proposed in the context of electronic mesoscopic systems (Datta, 1997; Imry and Landauer, 1999), where the energy and charge transport are mainly determined by the transmission function describing the transfer probability of the carriers. Moreover, techniques employed to compute transmission functions (scattering approaches, Green's function techniques, etc.) are conceptually very similar in all those contexts. This connection between RHT and conduction allows us not only to profit from the experience in other fields, but can also serve as the starting point for a unified description of different heat transfer modes in situations where different types of carriers may compete or even interfere. An example of this type of situation is realized in the context of the heat transfer in subnanometer gaps where recent experiments have reported conflicting observations in an intermediate regime where the contribution of different carriers (photons, phonons, and electrons) may be comparable (Cui et al. 2017a; Kloppstech et al., 2017). While the situation seems to be clear in the limiting cases where either conduction (Cui et al. 2019, 2017b; Mosso et al. 2017) or NFRHT (Kim et al., 2015 ) are clearly expected to dominate, the description of the crossover between them might require novel theories where different carriers are treated on an equal footing (Chiloyan et al., 2015, Venkataram et al. 2018).

Conceptually speaking, a major advance in the field in the last decade has been the development of theoretical models of RHT in many-body systems, the central topic of this review. Such a theory deals with radiative heat exchange in systems composed of multiple thermal emitters able to cooperatively interact. The collective behaviors in these systems give rise to singular phenomena that we discuss in the present manuscript. Until 2011, FE had been primarily used to describe RHT between two bodies, but the situation changed with the report of the first version of a many-body theory of RHT describing a collection of small dipolar particles (BenAbdallah et al. 2011). Soon after, this many-body theory was generalized to deal with bodies of arbitrary size and shape (Krüger et al., 2012, Messina and Antezza, 2011a), and new refinements of the theory are being constantly reported to deal with more complex optical materials. Again, there is here a clear analogy with developments in mesoscopic physics, where Büttiker's extension of the Landauer formalism to multi-terminal systems laid down the basis for the understanding of numerous charge and energy transport phenomena in mesoscopic systems (Datta, 1997). As we shall discuss in detail in this review, the many-body theory of NFRHT opened the door for predicting and analyzing a plethora of novel physical phenomena with no analogues in two-body systems. Thus, for instance, it became possible to explore thermal analogues of intrinsic many-body phenomena like the Hall effect (Ben-Abdallah, 2016) or heat persistent current (Zhu and Fan, 2016). It has also made it possible to propose a wide range of thermal functional devices that are intrinsically many-body in nature, such as the 
thermal transistor (Ben-Abdallah and Biehs, 2014). This theory also allowed for the first time to understand the different heat propagation regimes in disordered systems involving large collection of objects, and paved the way for hydrodynamic modelling of transport in these media. Although recent experimental works have explored the possibility to tune radiative heat transfers in many-body systems (Thompson et al. 2020) by actively changing the relative position of nearby objects, to our knowledge, many-body systems have yet to be experimentally investigated in the purely near-field regime.

The field of NFRHT has been the subject of different reviews over the years. Thus, for instance, the reviews by (Joulain et al., 2005) and (Volokitin and Persson, 2007) covered the FE theory and basic concepts of NFRHT, but for obvious reasons do not include crucial theoretical and experimental advances in recent years. The reviews by (Basu et al., 2007) and (Ben-Abdallah and Biehs, 2019) focus on potential applications of near-field thermal radiation in thermophotovoltaics. There are recent reviews like that of (Song et al., 2015a) that already presents some of the most recent advances and, in particular, describes the main experimental techniques developed in recent years. The review by (Cuevas and García-Vidal, 2018) provides an interesting and updated perspective of the field, but does not contain an in-depth description of theoretical developments. The present review article focuses on the theory of NFRHT in many-body systems, which has not been covered so far in a self-contained and unified framework. This topic is becoming a central focus of the field of thermal radiation, as it promises an entirely new generation of thermal radiation applications, and its understanding is likely to determine the future of RHT as a forefront research line.

The structure of the paper goes as follows. In Sec. II] we set the stage for this review by discussing NFRHT in two-body systems. Here, we put the emphasis on the modern view of NFRHT and review the most important theoretical advances in this topic, as well as the experimental state of the art. Specifically, we begin by briefly recalling the basics of the theory of $\mathrm{FE}$ and then discuss its application to the important case of two parallel plates (Sec. II.A). This basic configuration is used to illustrate the critical role of material choice (Sec. II.B, including a preliminary discussion of non-reciprocal materials in Sec. II.C. Section II.D is devoted to the analysis of the role of nanostructuring in tailoring and most importantly enhancing NFRHT, including recent works focused on multilayer structures, photonic crystals, metamaterials, gratings, metasurfaces, graphene sheets, and surface roughness. We then move beyond planar structures in Sec. II.E to discuss NFRHT between objects of arbitrary size and shape. General-purpose numerical methods developed so far for the description of NFRHT in arbitrary geometries are then discussed in Sec. II.F. We conclude this first part of the review in Sec. II.G with an in-depth discussion of recently derived limits on the largest NFRHT rates that could ever be realized by an optimal choice of material and geometric configuration. Specifically, we highlight the prohibitive role that multiple scattering (a critical feature of many-body physics to be further discussed in subsequent sections) plays in limiting heat-transfer enhancements that may be achieved through nanostructuring, resulting in optimal flux rates not much larger than what is observed in planar polaritonic materials, at least in the context of two-body heat exchange.

Section III constitutes the bulk of this review and covers a great variety of aspects of the theory of near-field thermal radiation in many-body systems. We first discuss the problem of light absorption by a set of non-emitting objects which collectively interact and show that these systems can be treated as a whole with a dressed susceptibility that takes into account both cooperative interactions as well as the resonant response of individual objects. Next, a generalized Landauer formula is derived to describe radiative heat transfer in the general situation in which all objects are emitting, using transmission coefficients describing the pairwise efficiency of coupling between any two objects. Using this theoretical framework, we highlight the singular aspects of heat transport in these systems compared to those seen in twobody systems. We start to illustrate these peculiarities in Secs. III.A.3 and III.B.2 where we prove the nonadditivity of heat flux, a fundamental feature of these systems. We also show that $N$-body interactions can amplify heat flux or lead to saturation mechanisms close to the contact without the need to introduce non-locality in material responsivity. In Sec. III.B.3, we discuss equilibrium conditions for any given system, and show that equilibrium states are generally not unique and can be, along with their stability, identified and characterized by standard perturbative techniques. We also show that multistable systems can be exploited, for instance, to make a boolean treatment of information with thermal photons or build thermal self-oscillators. In the subsequent section, we address the problem of heat transport in various complex systems using both a kinetic approach based on the approximate Boltzmann transport equation for the resonant modes supported by the system, and from a generalized Landauer theory that takes into account all modes in the continuum. Several physical effects (radiative drag effect, heat-flux focusing, heat pumping and long-range heat transport) inherent to many-body systems are then introduced and discussed. In Sec. III.C.4, we address the relaxation problem of many-body systems and show that the temperature field can evolve at different time scales, depending on the nature of interactions. Furthermore, we discuss the current solutions proposed to dynamically control the heat flux exchanged in these systems by modulating either geometrical configuration, optical properties, or via adiabatic control of their temperature. In Sec. III.C.6, we analyze various heat transport regimes in systems consisting of a large number of objects, and show that RHT can be described as a generalized random walk with a non-Gaussian probability 
distribution function. Unlike what happens in solid-state physics for heat conduction in bulk materials, we demonstrate the existence of anomalous heat transport regimes and highlight that these regimes closely depend on the system dimension, drastically changing from dilute to dense systems. The next few sections are devoted to nonreciprocal systems. Unlike reciprocal systems, in these non-Hermitian systems the classical notion of Lorentz reciprocity is violated, giving rise to specific heat-transfer mechanisms. After extending in Secs. III.D.2 and III.D.3 the theoretical framework to deal with heat exchange, we discuss in Secs. III.D.4 several thermomagnetic effects (magnetoresistance, permanent currents, Hall effect) that take place in magneto-optical systems and we underline in Sec. III.D.5 the link between these effects and the topological structure of the Poynting field. We also stress in Sec. III.D.7 the potential of these systems to efficiently tune the direction of heat flow. Finally, we conclude this review by listing outstanding challenges and a broader outlook of potential future research directions.

\section{TWO-BODY SYSTEMS}

Most theoretical work on the topic of NFRHT is primarily based on Rytov's FE theory. Developed in the 1950s (Rytov et al. 1989), FE is a semiclassical theory which assumes that thermal radiation is generated by random, thermally activated electric currents inside the bodies. Thus, the technical problem in the description of RHT between different objects boils down to the solution of the stochastic Maxwell's equations, with random electric currents as radiation sources. To illustrate the idea, let us consider two optically isotropic and non-magnetic bodies separated by a vacuum gap, see Fig. 2, In the framework of FE, the RHT problem is completely specified by the temperature distributions $T_{i}(\mathbf{r})(i=1,2)$ and the dielectric functions of the materials, $\epsilon_{i}(\mathbf{r}, \omega)$. The macroscopic Maxwell's equations to be solved adopt the following form in the frequency domain

$$
\begin{aligned}
\nabla \times \mathbf{E}(\mathbf{r}, \omega) & =i \omega \mu_{0} \mathbf{H}(\mathbf{r}, \omega), \\
\nabla \times \mathbf{H}(\mathbf{r}, \omega) & =-i \omega \epsilon_{0} \epsilon(\mathbf{r}, \omega) \mathbf{E}(\mathbf{r}, \omega)+\mathbf{J}(\mathbf{r}, \omega),
\end{aligned}
$$

where $\mathbf{E}$ and $\mathbf{H}$ are the electric and magnetic fields, $\mathbf{r}$ is the position vector, and $\epsilon_{0}$ and $\mu_{0}$ are the vacuum permittivity and permeability, respectively. In the second equation, the fluctuating current density distributions $\mathbf{J}(\mathbf{r}, \omega)$ within the bodies are the sources of the thermal radiation. The statistical average of these currents vanishes, i.e., $\langle\mathbf{J}\rangle=0$, but their correlations are given by the fluctuation-dissipation theorem (Eckhardt, 1984, Joulain et al., 2005, Rytov et al. 1989)

$$
\begin{aligned}
\left\langle\mathbf{J}(\mathbf{r}, \omega) \otimes \mathbf{J}^{*}\left(\mathbf{r}^{\prime}, \omega\right)\right\rangle= & \frac{4 \hbar \omega^{2} \epsilon_{0}}{\pi} \operatorname{Im}\{\epsilon(\mathbf{r}, \omega)\} \\
& \times n(\omega, T(\mathbf{r})) \delta\left(\mathbf{r}-\mathbf{r}^{\prime}\right),
\end{aligned}
$$

where $\hbar$ is the Planck constant and $n(\omega, T)=$ $1 /\left(\exp \left[\hbar \omega / k_{\mathrm{B}} T\right]-1\right)$ is the Bose function. In simple

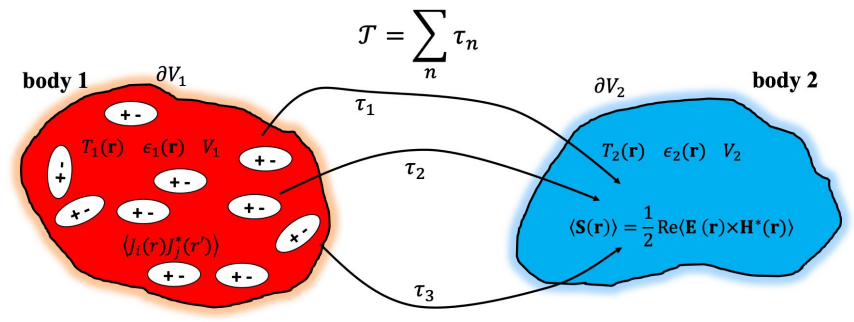

FIG. 2 Fluctuational electrodynamics: Schematic of radiative heat transfer in a two-body system. The two bodies of volumes $V_{1}$ and $V_{2}$ have temperature profiles $T_{1}(\mathbf{r})$ and $T_{2}(\mathbf{r})$ and frequency-dependent dielectric functions $\epsilon_{1}(\mathbf{r}, \omega)$ and $\epsilon_{2}(\mathbf{r}, \omega)$. Electromagnetic fields $\mathbf{E}$ and $\mathbf{H}$ are generated by the random currents $\mathbf{J}$ in the bodies due to their non-vanishing correlations given by the fluctuation-dissipation theorem. The net power exchanged by the two bodies is determined by the total transmission $\mathcal{T}$ that can be expressed as a sum of individual transmission coefficients $\tau_{n}$.

terms, the calculation of the radiative power exchanged by bodies 1 and 2 is done by first solving the Maxwell equations with the appropriate boundary conditions defined by geometries of the bodies and assuming that the random electric currents occupy the whole body 1 . Then, with the solution for the fields around body 2, the statistical average of the Poynting vector is computed: $\langle\mathbf{S}(\mathbf{r}, \omega)\rangle=\operatorname{Re}\langle\mathbf{E}(\mathbf{r}, \omega) \times \mathbf{H}(\mathbf{r}, \omega)\rangle / 2$. Finally, the results are integrated over frequency and over a closed surface enclosing body 2. Of course, to evaluate the net RHT, one needs to calculate in a similar way the heat transferred from body 2 to body 1 .

This innocent-looking problem is, however, quite challenging in general, and analytical solutions are only known in a handful of situations. One of the main goals of the rest of this section is to present the solution in cases of increasing complexity focusing on two-body systems. Let us say at this stage that, as mentioned in the introduction, the net power, $P_{\text {net }}$, exchanged via thermal radiation between two objects of (homogeneous) temperatures $T_{1}$ and $T_{2}$ can always be expressed via means of the Landauer formula, as one can easily understand with the following heuristic argument. The net radiative power is the balance between the heat power transferred from one body to the other: $P_{\text {net }}=P_{1 \rightarrow 2}-P_{2 \rightarrow 1}$, where the individual contributions are given by

$$
\mathcal{P}_{i \rightarrow j}=\int_{0}^{\infty} \frac{d \omega}{2 \pi} \hbar \omega n\left(\omega, T_{i}\right) \mathcal{T}_{j i}(\omega) .
$$

Here, $\hbar \omega$ is the energy of an electromagnetic mode of frequency $\omega$ and the Bose function $n(\omega, T)$ is describing the thermal occupation of that mode, and $\mathcal{T}_{j i}(\omega)$ is the total transmission coefficient that correspond to the sum of the probabilities over all the modes of frequency $\omega$ that can be transferred from body $i$ to body $j$. In the case of a two-body system (with no environment), detailed balance imposes that $\mathcal{T}_{21}(\omega)=\mathcal{T}_{12}(\omega)=\mathcal{T}(\omega)$ and the expression of the net power reduces to the cel- 
ebrated Landauer formula Ben-Abdallah and Joulain, 2010 Biehs and Greffet, 2010a: Polder and Hove, 1971)

$$
\mathcal{P}_{\text {net }}=\int_{0}^{\infty} \frac{d \omega}{2 \pi} \hbar \omega\left[n\left(\omega, T_{1}\right)-n\left(\omega, T_{2}\right)\right] \mathcal{T}(\omega) .
$$

Following the spirit of the Landauer approach in mesoscopic physics, the total transmission can be analyzed in terms of radiation channels and it can be expressed as

$$
\mathcal{T}(\omega)=\sum_{n} \tau_{n}(\omega)
$$

where the $\tau$ 's are the individual transmission probabilities of the different open channels (bounded between 0 and 1). This point is particularly useful to establish simple upper bounds for RHT, as we shall discuss later in this review.

\section{A. Parallel plates}

As mentioned in the introduction, the importance of the contribution of evanescent waves in the RHT between two objects and the possibility to overcome the Planckian limit in the near-field regime was first put forward by Polder and van Hove (Polder and Hove, 1971). These authors calculated the NFRHT rate between two infinite parallel plates, a geometry that has become the workhorse of NFRHT and that is schematically represented in Fig. 1. We shall refer to the upper plate as medium 1 and the lower plate as 2, and assume that they are at constant temperatures $T_{1}$ and $T_{2}$, respectively. In the case of optically isotropic and nonmagnetic materials, Polder an Van Hove showed that the radiative power per unit area, i.e. the heat flux $\Phi$, between the parallel plates is given by Eq. (5) with the following replacement of the transmission coefficient by a transmission coefficient per unit area:

$$
\mathcal{T}(\omega) \longrightarrow \int_{0}^{\infty} \frac{d \kappa}{2 \pi} \kappa \tau(\omega, \kappa, d) .
$$

Here, $\kappa=\sqrt{k_{x}^{2}+k_{y}^{2}}$ is the magnitude of the wave vector parallel to the plates, see coordinate system in Fig. 11(a), $d$ is the gap size, and $\tau(\omega, \kappa, d)$ is the total (sum over polarizations) transmission probability of an electromagnetic mode of frequency $\omega$ and parallel wave vector $\kappa$. In the case of isotropic materials, this total transmission is equal $\tau(\omega, \kappa, d)=\tau_{s}(\omega, \kappa, d)+\tau_{p}(\omega, \kappa, d)$, where the contributions of $s$ - and $p$-polarized waves (or alternatively TE- and TM-waves) are given by $(\alpha=s, p)$

$$
\tau_{\alpha}(\omega, \kappa, d)= \begin{cases}\frac{\left(1-\left|r_{1}^{\alpha}\right|^{2}\right)\left(1-\left|r_{2}^{\alpha}\right|^{2}\right)}{\left|D^{\alpha}\right|^{2}}, & \kappa<k_{0} \\ \frac{4 \operatorname{Im}\left(r_{1}^{\alpha}\right) \operatorname{Im}\left(r_{2}^{\alpha}\right) e^{-2\left|q_{\mathrm{v}}\right| d}}{\left|D^{\alpha}\right|^{2}}, & \kappa>k_{0},\end{cases}
$$

where $k_{0}=\omega / c$ is the wavenumber in vacuum and $D^{\alpha}=$ $1-r_{1}^{\alpha} r_{2}^{\alpha} e^{2 i q_{\mathrm{v}} d}, c$ is the speed of light, $q_{\mathrm{v}}=\sqrt{\omega^{2} / c^{2}-\kappa^{2}}$ is the perpendicular component of the wave vector in the vacuum gap, and $r_{i}^{\alpha}$ are Fresnel (or amplitude reflection) coefficients given by

$$
r_{i}^{s}=\frac{q_{\mathrm{v}}-q_{i}}{q_{\mathrm{v}}+q_{i}}, \quad r_{i}^{p}=\frac{\epsilon_{i} q_{\mathrm{v}}-q_{i}}{\epsilon_{i} q_{\mathrm{v}}+q_{i}} .
$$

Here, $\epsilon_{i}(\omega)$ is the dielectric function of medium $i=1,2$, assumed to only depend on frequency (local media), and $q_{i}=\sqrt{\epsilon_{i} k_{0}^{2}-\kappa^{2}}$.

The key point in this result is that the integral in Eq. (7) is carried out over all possible values of $\kappa$ and therefore, it includes the contribution of both propagating waves $\left(\kappa<k_{0}\right)$ and evanescent waves $\left(\kappa>k_{0}\right)$. These latter ones are not taken into account in StefanBoltzmann's law. The contribution of the evanescent waves decays exponentially with the gap size, see Eq. (8), and it becomes negligible in the far-field regime $(d \gg$ $\left.\lambda_{\mathrm{Th}}\right)$. However, in the near-field regime $\left(d<\lambda_{\mathrm{Th}}\right)$ the contribution of evanescent waves, often referred to as photon tunneling, can become very significant and for sufficiently small gaps, it may completely dominate the heat transfer. The black-body result is obtained from Eq. (7) by ignoring the evanescent waves and assuming perfect transmission for the propagating waves for all frequencies and wave vectors. In that case, the radiative power per unit area is given by Stefan-Boltzmann's law: $\Phi_{\mathrm{BB}}=\sigma\left(T_{1}^{4}-T_{2}^{4}\right)$, where $\sigma=5.67 \times 10^{-8} \mathrm{~W} /\left(\mathrm{m}^{2} \mathrm{~K}^{4}\right)$.

\section{B. Metals vs. dielectrics}

The parallel-plate configuration allows us to illustrate not only the impact of evanescent waves in the near-field regime, but also the importance of the choice of materials. There are two main classes of materials when it comes to NFRHT, namely metals (or related materials with free carriers like doped semiconductors) and dielectrics (especially polar dielectrics that exhibit polaritonic resonances like $\mathrm{SiO}_{2}, \mathrm{SiN}, \mathrm{SiC}$, etc.). As an example of the results for these two types of materials, we show in Fig. $3(\mathrm{a}, \mathrm{c})$ the gap dependence of the room-temperature heat-transfer coefficient, i.e. the radiative heat conductance per unit area, for two parallel plates made of $\mathrm{Au}$ and $\mathrm{SiO}_{2}$. In those panels we also show the individual contributions of propagating and evanescent waves for TE and TM polarizations. Notice that in both cases the Planckian limit (indicated with an horizontal line) is greatly overcome for sufficiently small gaps. This is particularly remarkable in the silica case, where for $d=1 \mathrm{~nm}$ the heat flux is almost 5 orders of magnitude larger than the black-body limit. Notice also that there are clear differences between $\mathrm{Au}$ and $\mathrm{SiO}_{2}$. For $\mathrm{Au}$, the NFRHT rate is dominated by TE evanescent waves, which originate from eddy currents inside the Au plates (Chapuis et al. 2008c). This typically leads to a saturation of the heat transfer coefficient for small gaps. On the contrary, in the silica case, NFRHT is dominated by TM evanescent waves that can be shown 

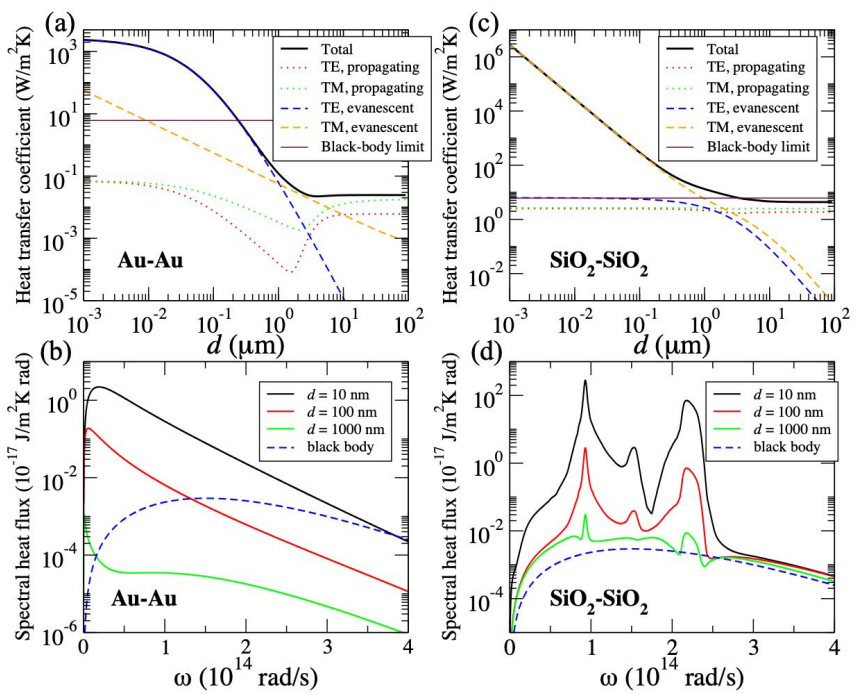

FIG. 3 (a) Heat transfer coefficient at room temperature $(300 \mathrm{~K})$ as a function of the gap size for two infinite parallel plates made of Au. The different lines correspond to the total contribution (black solid line) and to the contributions of propagating and evanescent waves for TE and TM polarizations. The horizontal line shows the result for two black bodies: $6.124 \mathrm{~W} /\left(\mathrm{m}^{2} \mathrm{~K}\right)$. (b) The spectral heat flux (or conductance per unit area and frequency) as a function of the radiation frequency corresponding to the case of panel (a). The solid lines correspond to three different values of the gap size in the near-field regime, while the blue dashed line is the result for two black bodies. (c,d) The same as in panels $(\mathrm{a}, \mathrm{b})$ for $\mathrm{SiO}_{2}$.

to stem from surface phonon polaritons (SPhPs): quasiparticle excitations that arise from the strong coupling of electromagnetic fields with the optical phonon modes of polar dielectrics (Mulet et al. 2002). These surface electromagnetic waves are hybrid or cavity modes that reside in both plates and have a penetration depth that is on the order of the gap size (Basu and Zhang, 2009), which implies that they are more and more confined to the surfaces as the gap is reduced (Song et al. 2015b). The increase of the density of the states of theses modes (BenAbdallah and Joulain, 2010, Biehs and Greffet, 2010a) upon reducing the gap size is reflected in a characteristic $1 / d^{2}$ dependence of the heat transfer coefficient for polar dielectrics.

Apart from enhancing NFRHT, evanescent waves are also responsible for a drastic modification of the spectral heat flux (or heat conductance per unit frequency), see Fig. 3(b,d). Thus, for instance, in the $\mathrm{SiO}_{2}$ case the spectral heat flux is dominated by two peaks that appear at the frequencies of the optical modes of this polar dielectric. This is dramatically different as compared to the broadband Planck's distribution and it is also due to the fact that NFRHT in this case is dominated by SPhPs.

In principle, the plate-plate configuration discussed above is ideally suited to experimentally investigate NFRHT because some of the largest enhancements in this
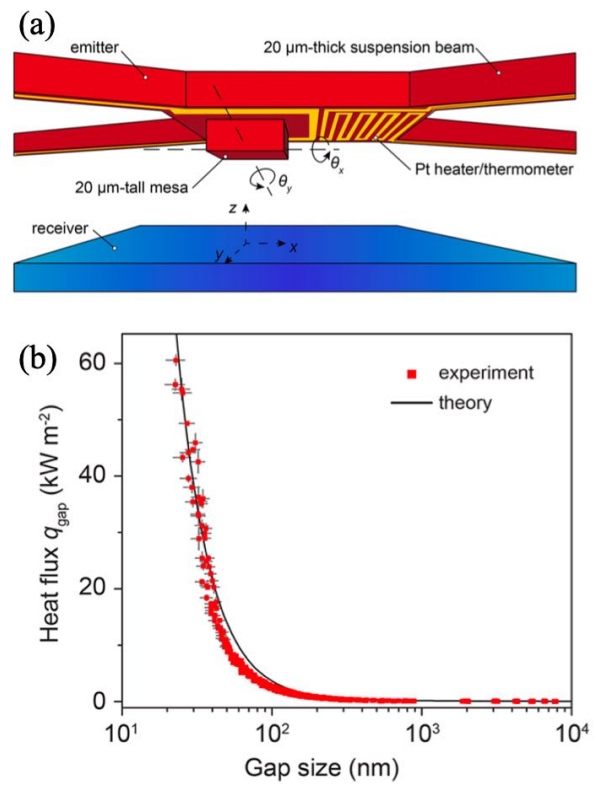

FIG. 4 (a) Schematic illustration of NFRHT measurement configuration used by (Fiorino et al. 2018b). The emitter microdevice is comprised of a square mesa and $\mathrm{Pt}$ heater/thermometer suspended on a thermally isolated island. The receiver is a macroscopically large $(1 \mathrm{~cm} \times 1 \mathrm{~cm})$ plate. (b) The corresponding heat flux versus gap size in the case of an emitter and an receiver made of $\mathrm{SiO}_{2}$. Measured data (red squares) is compared to the theoretical result (solid black line) obtained within FE. Reprinted with permission from (Fiorino et al. 2018b). Copyright 2018 ACS.

regime are expected to occur in this setting. However, this configuration is very difficult to realize in practice because it is very complicated to achieve and maintain good parallelism between macroscopic plates at nanometer separations. In recent years several groups have overcome this hurdle and have developed novel techniques to explore the plate-plate configuration in the near-field regime and they have been able to confirm the results of the FE theory. Some of those experiments have made use of macroscopic $(\sim \mathrm{cm} \times \mathrm{cm})$ planar surfaces Bernardi et al. 2016: DeSutter et al., 2019, Ghashami et al., 2018; $\mathrm{Hu}$ et al., 2008, Ottens et al., 2011), while others are based on microscopic plates $(50 \mu \mathrm{m} \times 50 \mu \mathrm{m})$ (Fiorino et al. 2018b; Song et al., 2016, St-Gelais et al. 2014, 2016). The use of macroscopic planar surfaces is conceptually simple, but in practice it is more difficult to ensure the parallelism and to have clean and smooth surfaces over such large areas. For this reason, the smallest gaps achieved with this strategy are still above a hundred nanometers (DeSutter et al., 2019). On the other hand, the use of microdevices facilitates the parallelization of the systems and the characterization of the surfaces. With this approach, it has become possible to explore gaps as small as $30 \mathrm{~nm}$ (Fiorino et al. 2018b), as we illustrate in Fig. 4. In this example, a microdevice comprising a Pt resistor, which heats up the emitter and measures its temperature, was used to measure the 
NFRHT rate between two $\mathrm{SiO}_{2}$ surfaces down to gaps of about $30 \mathrm{~nm}$. For these tiny gaps, it was found that the heat conductance was about 1200 times larger than in the far-field regime and about 700 times larger than the black-body limit, in excellent agreement with the theory results based on FE.

\section{Non-reciprocal materials}

A special class of materials that has attracted a lot of attention in the context of thermal radiation is that of non-reciprocal materials. These materials do not satisfy Lorentz reciprocity (Caloz et al. 2018) and, in practice, are optically anisotropic materials with dielectric tensors which are non-symmetric. A paradigmatic example is that of magneto-optical (MO) materials where the non-reciprocity is induced either by an internal magnetization like in ferromagnets or by an external magnetic field like in doped semiconductors. Part of the attention is due to the suggestion that these materials might violate Kirchhoff's law (Zhu and Fan, 2014), which establishes the equality of thermal emissivity and absorptivity. Although it has been shown that this is not case in a two-body situation (one body could be an environment) (Ekeroth et al. 2017), this class of materials does give rise to countless novel thermal-radiation phenomena in the context of many-body systems, as it will be amply discussed later in this review.

In the context of NFRHT in two-body non-reciprocal systems, most of the work so far has focused on the analysis of MO materials and, in particular, on the study of the use of an external magnetic field as a way to actively control thermal radiation. Special attention has been devoted to doped semiconductors, which in the presence of an external magnetic field exhibit a very strong MO activity in the infrared. The first theoretical study of this kind was reported by (Moncada-Villa et al., 2015) who analyzed the magnetic-field dependence of the heattransfer coefficient of two parallel plates made of doped semiconductors ( $\mathrm{InSb}$ or $\mathrm{Si}$ ). These materials become optically anisotropic and non-reciprocal in the presence of an external magnetic field. Thus, the problem is to compute the RHT between between two anisotropic parallel plates. This generic problem was addressed by (Biehs et al., 2011, Bimonte, 2009) and, similarly to the isotropic case discussed in Sec. II.A the net power per unit area or heat flux $\Phi$ is given by the Landauer formula of Eq. (5) with the substitution

$$
\mathcal{T}(\omega) \longrightarrow \int \frac{d \boldsymbol{\kappa}}{(2 \pi)^{2}} \tau(\omega, \boldsymbol{\kappa}, d) .
$$

Here, $\boldsymbol{\kappa}=\left(k_{x}, k_{y}\right)^{t}$ is the wave vector parallel to the surface planes, and $\tau(\omega, \boldsymbol{\kappa}, d)$ is the transmission probability of the individual electromagnetic waves. Notice that the integral in Eq. (10) is now carried out over all possible directions of $\boldsymbol{\kappa}$ (the RHT is no longer isotropic) and, as usual, it includes the contribution of both propagat- ing and evanescent waves. The transmission coefficient $\tau(\omega, \boldsymbol{\kappa}, d)$ can be expressed as

$$
\begin{aligned}
& \tau(\omega, \kappa, d)= \\
& \begin{cases}\operatorname{Tr}\left\{\left[\mathbb{1}-\mathbb{R}_{1} \mathbb{R}_{1}^{\dagger}\right] \mathbb{D}^{\dagger}\left[\mathbb{1}-\mathbb{R}_{2}^{\dagger} \mathbb{R}_{2}\right] \mathbb{D}\right\}, & \kappa<k_{0} \\
\operatorname{Tr}\left\{\left[\mathbb{R}_{1}-\mathbb{R}_{1}^{\dagger}\right] \mathbb{D}^{\dagger}\left[\mathbb{R}_{2}^{\dagger}-\mathbb{R}_{2}\right] \mathbb{D}\right\} e^{-2\left|q_{\mathrm{v}}\right| d}, & \kappa>k_{0}\end{cases}
\end{aligned}
$$

where the $2 \times 2$ matrices $\mathbb{R}_{i}$ (with $i=1,2$ ) are the reflection matrices characterizing the two interfaces. These matrices have the following generic structure

$$
\mathbb{R}_{i}=\left(\begin{array}{cc}
r_{i}^{s s} & r_{i}^{s p} \\
r_{i}^{p s} & r_{i}^{p p}
\end{array}\right)
$$

where $r_{i}^{\alpha \beta}$ with $\alpha, \beta=s, p$ is the reflection amplitude for the scattering of an incoming $\alpha$-polarized plane wave into an outgoing $\beta$-polarized wave. In particular, the offdiagonal elements describe the polarization conversation, which does not occur for isotropic materials. Finally, the $2 \times 2$ matrix $\mathbb{D}$ in Eq. (11) is defined as

$$
\mathbb{D}=\left[\mathbb{1}-\mathbb{R}_{1} \mathbb{R}_{2} e^{2 i q_{\mathrm{v}} d}\right]^{-1} .
$$

The different reflection matrices appearing in Eq. (12) can be computed within standard approaches for anisotropic multilayer systems.

This formalism was used in (Moncada-Villa et al. 2015) to show that the NFRHT rate between two parallel plates made of doped InSb and Si can be strongly affected by the application of a static magnetic field, and relative changes of up to $700 \%$ were predicted for fields of a few Teslas. These results are illustrated in Fig. 5 for the case of a magnetic field oriented parallel to the plates. More recently, the same authors have also shown that NFRHT between two parallel plates made of MO materials can also be modulated by simply changing the orientation of the external magnetic field (Moncada-Villa and Cuevas, 2020), which is the thermal analogue of wellknown phenomenon of anisotropic thermal magnetoresistance in the field of spintronics. This and other thermomagnetic phenomena in the context small MO particles will be discussed in more detail in Sec. III.D,

\section{Nanostructuring and Roughness}

Following ideas and concepts of nanophotonics, many groups have explored nanostructuring as a strategy to further enhance NFRHT and to tune its spectral properties. In this subsection, we shall briefly review some of the ideas put forward in recent years in the context of NFRHT in nanostructured planar systems and also discuss the impact of deviations from planarity.

\section{Multilayer structures and photonic crystals}

A natural extension of the plate-plate configuration discussed above is to replace the plates by planar multi- 


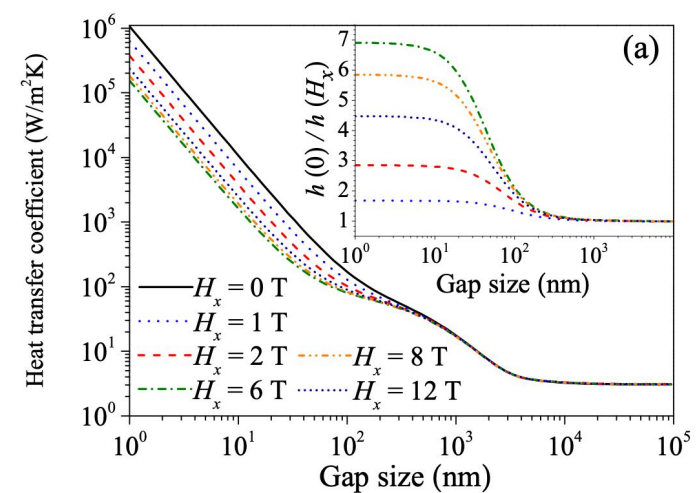

Wavelength $(\mu \mathrm{m})$

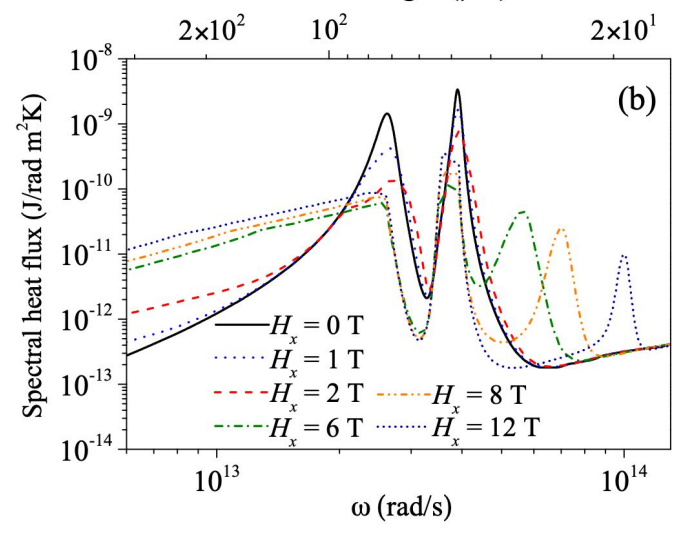

FIG. 5 (a) Heat-transfer coefficient for two parallel plates made of $n$-doped $\mathrm{InSb}$ at room temperature $(300 \mathrm{~K})$ as a function of the gap size for different values of a magnetic field applied parallel to the surfaces of the plates ( $x$-direction). The inset shows the ratio between the zero-field coefficient and the coefficient for different values of the field in the nearfield region. (b) The corresponding spectral heat flux as a function of the frequency (and wavelength) for a gap of $d=10$ $\mathrm{nm}$ and different values of the parallel field. Reprinted with permission from (Moncada-Villa et al. 2015). Copyright 2015 American Physical Society.

layer structures or 1D photonic crystals (Basu and Francoeur, 2011, Ben-Abdallah et al., 2009a b, 2010||Biehs, 2007; Biehs et al., 2007, Francoeur et al. 2008, 2010a, 2011; Iizuka and Fan, 2018; Jin et al., 2017a; Maslovski et al. 2013; Miller et al. 2014). A central idea in this case is to incorporate thin films in layered systems to make better use of surface electromagnetic modes. In practice, the RHT rate between two planar multilayer bodies comprised of an arbitrary number of layers can be formally described with the same formulas as in the plate-plate case, see Eqs. (7) and (8), but in this case $r_{1}^{\alpha}$ and $r_{2}^{\alpha}$ have to be interpreted as the reflection coefficients of the two subsystems (including their complete layered structures), see (Ben-Abdallah et al. 2010 , Bimonte, 2009). To give a concrete example, let us follow (Song et al. 2015b) and consider the multilayer structure shown in the inset of Fig. 6 where the first body is an infinite $\mathrm{SiO}_{2}$ plate (medium 1) and the second body features a $\mathrm{SiO}_{2}$ film of thickness $t$ (medium 3) deposited on a semi-infinite layer of $\mathrm{Au}$ (medium 4), while the medium 2 is the vacuum gap of size $d$. In this case, $r_{2}^{\alpha}$ in Eq. (8) has to be replaced by (Biehs, 2007)

$$
R^{\alpha}=\frac{r_{23}^{\alpha}+r_{34}^{\alpha} e^{2 i q_{3} t}}{1-r_{34}^{\alpha} r_{32}^{\alpha} e^{2 i q_{3} t}}
$$

which is the reflection coefficient of the subsystem formed by media 3 and 4 . Here, as usual, the $r_{i j}^{\alpha}$ are the Fresnel coefficients of the different interfaces:

$$
r_{i j}^{s}=\frac{q_{i}-q_{j}}{q_{i}+q_{j}} \text { and } r_{i j}^{p}=\frac{\epsilon_{j} q_{i}-\epsilon_{i} q_{j}}{\epsilon_{j} q_{i}+\epsilon_{i} q_{j}},
$$

where $q_{i}=\sqrt{\epsilon_{i} k_{0}^{2}-\kappa^{2}}$. Finally, the Fabry-Pérot denominator in Eq. (8) adopts now the form $D^{\alpha}=1-$ $r_{21}^{\alpha} R^{\alpha} e^{2 i q_{2} d}$.

In Fig. 6 we show representative results of the gap dependence of the-heat transfer coefficient of this multilayer structure for different values of the thickness of the silica film, ranging from $50 \mathrm{~nm}$ to bulk. We also show the result with no $\mathrm{SiO}_{2}$ film for comparison. Notice that for small gaps $(d<100 \mathrm{~nm})$, the results are independent of the silica film thickness, which shows that the extraordinary NFRHT enhancements that occur in the bulk systems made of polar dielectrics are also possible in thin-film structures as long as the gap size is smaller than the film thickness (Biehs, 2007, Biehs et al. 2007). As explained above, the physical origin of these results can be traced back to the fact that NFRHT is dominated by electromagnetic cavity modes arising from SPhPs whose penetration depth scales with the gap size. Thus, when the gap is sufficiently small, all the heat transfer comes from a shallow region on the surface of the two bodies and NFRHT becomes independent of the film thickness. These qualitative predictions were subsequently experimentally confirmed by (Song et al. 2015b) using a 53$\mu \mathrm{m}$-diameter silica sphere as an emitter, instead of the silica plate used in the calculations of Fig. 6. The finite curvature of the sphere results in smaller NFRHT enhancements, as compared to the planar structure, as it is easy to understand with the standard proximity approximation, see (Song et al., 2015b) for details.

To increase NFRHT beyond bulk systems, different groups have proposed to combine several thin films to make use of the hybridization of the surface modes in different interfaces (Ben-Abdallah et al., 2009a; Biehs, 2007; Francoeur et al., 2008, 2011; Iizuka and Fan, 2018; Jin et al. 2017a). Another proposed strategy to outperform bulk systems relies on the use of $1 \mathrm{D}$ photonic crystals (Ben-Abdallah et al., 2010; Tschikin et al., 2012a). In this case the heat transfer mechanism involves the surface Bloch states coupling supported by these media.

\section{Metamaterials}

Another topic that has been extensively studied in the context of NFRHT between nanostructured systems is 


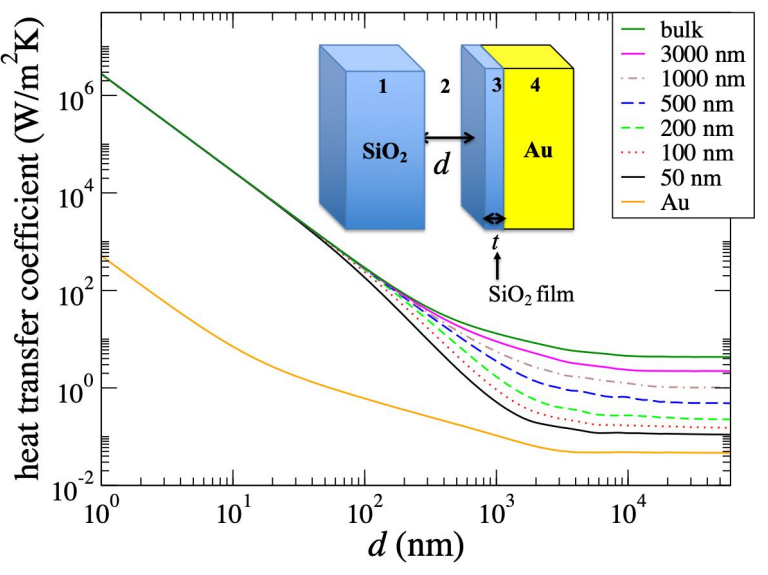

FIG. 6 Computed heat transfer coefficient as a function of gap size for the multilayer system shown in the inset at room temperature $(300 \mathrm{~K})$. This structure comprises a thick, semiinfinite silica surface separated by a vacuum gap of size $d$ from a silica thin film coating on a semi-infinite Au surface. Different curves correspond to different thicknesses of the silica coating. Adapted from (Song et al., 2015b).

the use of metamaterials, i.e., artificial structures with subwavelength features designed to exhibit complex optical properties that are difficult to find in naturally occurring (bulk) materials. In particular, special attention has been devoted to hyperbolic metamaterials, which are a special class of highly anisotropic media whose electromagnetic modes have an hyperbolic dispersion relation. To be precise, they are uniaxial materials for which one of the principal components of either the permittivity or the permeability tensor is opposite in sign to the other two principal components. These systems have been primarily fabricated based on designs involving hybrid metal-dielectric superlattices and metallic nanowires embedded in dielectric hosts (Poddubny et al., 2013). The interest in these metamaterials in the context of NFRHT lies in the fact that they have been predicted to behave as broadband super-Planckian thermal emitters (Biehs et al., 2012; Guo et al., 2012, Nefedov and Simovski, 2011). This behavior originates from the fact that these metamaterials can support electromagnetic modes that are evanescent in a vacuum gap, but which are propagating inside the material. This leads to broadband enhancement of the transmission efficiency of the evanescent modes (Biehs et al. 2012). From the computational point of view, the heat transfer between hyperbolic metamaterials can be described using either the scattering approach for multilayer media described in the previous subsection, or the more general method discussed in the following subsection and applicable to laterally periodic patterned structures. In this latter case, and for appropiate (subwavelength) periodicities, it is typical to expoit an effective medium theory in order to reduce the problem to one involving planar but optically anisotropic materials, allowing application of the approach described in Sec. II.C.
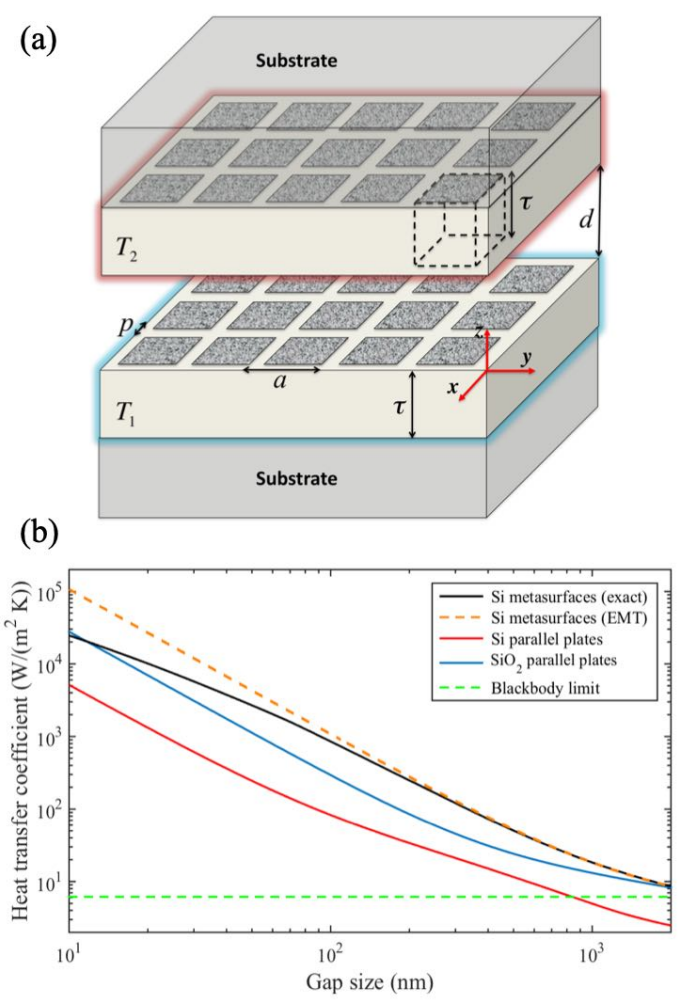

FIG. 7 (a) Schematic of two doped-Si metasurfaces made of $2 \mathrm{D}$ periodic arrays of square holes placed on semi-infinite planar substrates and held at temperatures $T_{1}$ and $T_{2}$. (b) Room-temperature heat transfer coefficient as a function of the gap size for the doped-Si metasurfaces of panel (a) with $a=50 \mathrm{~nm}$ and a filling factor of 0.9 (black line). For comparison, the plot also includes the results for the Si metasurfaces computed with an effective medium theory (orange dashed line), $\mathrm{SiO}_{2}$ parallel plates (blue line), and doped-Si parallel plates (red line). The horizontal dashed line shows the blackbody limit. Reprinted with permission from ref (FernándezHurtado et al. 2017). Copyright 2017 by the APS.

The special properties of hyperbolic metamaterials have spurred many theoretical investigations of their use in the context of NFRHT (Biehs et al., 2013, Guo and Jacob, 2013, 2014 Lang et al. 2015 Liu et al., 2013, 2014b; Miller et al., 2014: Simovski et al., 2013 Tschikin et al. 2015, 2013). These works have in turn demonstrated that metamaterials do not outperform thin-film-based structures exhibiting $\mathrm{SPhPs}$, as their increased density of states is compensated for by a decrease in the strength of the evanescent fields (Miller et al., 2014). Nevertheless, metamaterials exhibit other interesting properties; for instance, the long penetration depth of the hyperbolic modes can be advantageous for applications in near-field thermophotovoltaics.

\section{Gratings and Metasurfaces}

Also inspired by nanophotonic concepts, NFRHT between periodically patterned systems has been intensively 
investigated from a theoretical point of view, both in 1D (gratings) and in 2D (photonic crystals and periodic metasurfaces). Again, the goal of such nanostructuring is to tune the spectral heat transfer and enhance net NFRHT. Technically speaking, the Landauer formula of the previous subsections can be straightforwardly generalized to deal with periodic systems by making use of Bloch's theorem. This was first done by Bimonte and we refer to (Bimonte, 2009) for technical details. Using that generalized formula in combination with different techniques for the computation of reflection coefficients in periodic systems, typically via the rigorous coupled wave analysis (RCWA) method, several groups have reported calculations of NFRHT between periodic metallic nanostructures in both 1D (Dai et al., 2016a, 2015; Guérout et al., 2012, Messina et al., 2017b) and 2D (Dai et al. 2016b; Jin et al., 2019). The key idea in this case is to use nanostructuring to create new surface modes, referred to as spoof plasmons (Pendry et al., 2004), whose frequencies can be adjusted by tuning the length scales of these periodic systems so that their surface modes can be thermally populated at the desired working temperature. The reported results have clearly demonstrated the possibility of enhancing NFRHT over the corresponding planar (bulk) materials. However, NFRHT in these periodically patterned metallic structures continues to be smaller than that observed in simple (unstructured) planar polar dielectrics, with few exceptions (Jin et al. 2019).

There has also been significant theoretical work on the topic of NFRHT between dielectric photonic crystals and metasurfaces (Liu and Zhang, 2015a, Liu et al. 2015. Rodriguez et al. 2011). Again, these structured systems exhibit enhanced NFRHT with respect to their bulk counterparts, but the resulting NFRHT rates are again much smaller than those of planar polar dielectrics. In this regard, it is worth mentioning that it has been predicted that metasurfaces can indeed provide a way to enhance NFRHT between extended structures (FernándezHurtado et al., 2017). To be precise, it has been shown that Si-based metasurfaces featuring two-dimensional periodic arrays of holes, see Fig. 7. can exhibit a roomtemperature near-field radiative heat conductance larger than any unstructured material to date. This enhancement relies on the possibility of largely tuning the spectral properties of the surface plasmon polaritons that dominate NFRHT in these structures. In particular, nanostructuring enables the appearance of broadband and lower-frequency surface modes, increasing their contribution and occupation at room temperature, which constitutes one of the main strategies being pursued to enhance NFRHT. We conclude this subsection by noting that, to our knowledge, no experiment has thus far probed NFRHT between patterned structures.

\section{Graphene}

Two-dimensional materials are revolutionizing material science and they also hold promise in the field of NFRHT. In particular, graphene has attracted much attention as it can support delocalized surface plasmon polaritons (SPPs) that can contribute to NFRHT in spite of graphene's ultrasmall (one-atom) thickness (Ilic et al. 2012a: Volokitin and Persson, 2011). What makes these surface modes so attractive, as compared to SPhPs in polar dielectrics, is the possibility of modulating them electronically (Messina et al. 2013a), which can be achieved by controlling graphene's chemical potential by means of a nearby gate electrode. Such a mechanism provides an ideal strategy to actively control NFRHT in graphenebased structures (Papadakis et al. 2019). On the other hand, several theoretical studies have shown that coating structures with graphene sheets may lead to a substantial increase in NFRHT (Lim et al. 2013; Messina et al., 2017a; Svetovoy et al. 2012). In this case, the idea is that appropriate engineering of the coupling of graphene's SPPs with other surface modes, like SPPs in doped Si or SPhPs in polar dielectrics, may increase the efficiency of heat exchange in the near-field regime. Another topic of great interest that has been theoretically investigated is the use of graphene-based structures in thermophotovoltaics (Ilic et al., 2012b; Messina and BenAbdallah, 2013: Svetovoy and Palasantzas, 2014). Furthermore, the role of graphene in NFRHT has been theoretically studied in a wide variety of hybrid structures (Liu et al., 2014b; Liu and Zhang, 2015b, Shi et al., 2017. 2018, 2019b Zhao et al. 2017b).

From an experimental perspective, recent works have confirmed that graphene enables enhanced NFRHT between polar dielectrics (Shi et al., 2019a, van Zwol et al., 2012b) and between Si substrates (both insulating and conductive) (Yang et al., 2018). In particular, Shi et al. (Shi et al., 2019a) measured the NFRHT flux between two identical graphene-coated $\mathrm{SiO}_{2}$ heterostructures with millimeter-scale surface area and reported a 64-fold enhancement compared to the corresponding black-body limit for a gap size of $170 \mathrm{~nm}$, see Fig. 8 . Moreover, these authors showed theoretically that the physical mechanism behind this large NFRHT enhancement is indeed the coupling between graphene's SPPs and silica's SPhPs. It is also worth mentioning that the first experimental demonstration of NFRHT modulation by electronic gating of a graphene field-effect heterostructure was just recently reported (Thomas et al. 2019).

\section{Surface roughness}

Most calculations of NFRHT in planar structures assume that the corresponding surfaces are perfectly flat. Such an idealization, for instance, ignores practical considerations such as surface roughness. The impact of surface roughness on NFRHT was addressed theoreti- 


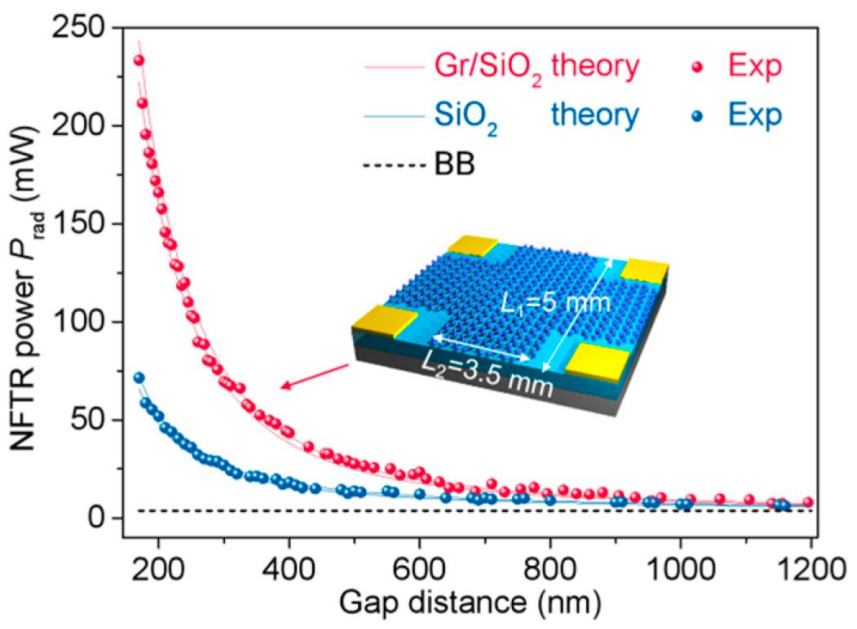

FIG. 8 Comparison of the NFRHT rate between graphene(Gr)/ $\mathrm{SiO}_{2}$ pair (red-solid line) and $\mathrm{SiO}_{2}$ pair (blue-solid line) with various gap sizes. The temperatures of the emitter and the receiver are $323.2 \mathrm{~K}$ and 301.5, respectively. Lines show the calculated values and spheres are the average values of four repeated measurements at each point. The inset shows a schematic illustration of the $\mathrm{Gr} / \mathrm{SiO}_{2}$ heterostructure. The black-body limit has been plotted for comparison (black-dashed line). Reprinted with permission from (Shi et al. 2019a). Copyright 2019 ACS.

cally by Biehs and Greffet (Biehs and Greffet, 2010b), in a plate-plate configuration. Using a form of perturbation theory, they showed that assuming reasonable values for the height of the roughness profile $(\sim 5 \mathrm{~nm})$, corrections to the heat transfer coefficient due to roughness can lead to roughly order of magnitude differences compared to perfectly flat surfaces when the gap size is on the order of a few tens of $\mathrm{nm}$, both for metals and polar dielectrics. Moreover, they showed that proximity approximations previously used for describing rough surfaces are highly innacurate when gap sizes become much smaller than the correlation length of the surface roughness, even when the heat transfer is dominated by the coupling of surface modes. We also note that the influence of surface roughness has also been studied by way of the finite-difference time-domain method in combination with the Wiener chaos expansion approach (Chen and Xuan, 2015), along with its interplay with surface curvature (Krüger et al., 2013).

\section{E. Impact of geometry}

Thus far, we have mainly discussed NFRHT in planar geometries in which the translational symmetry greatly simplifies the resolution of Maxwell's equations. In what follows, we turn to the analysis of the impact of geometry (heat exchange between structured bodies) and briefly discuss how the aforementioned RHT formulas can be generalized to handle objects of arbitrary size and shape.

The Polder-van Hove formula expressing $\mathcal{T}(\omega)$ in terms of Fresnel reflection coefficients or generalized reflection matrices is well-suited for calculations of heat transfer in systems with translational symmetry, including the aforementioned uniform planar slabs, thin films, gratings, photonic crystals, and periodic metamaterials. However, this leaves out a large class of systems of experimental and theoretical interest that do not exhibit such translational symmetries, particularly compact bodies like spheres or structured nanoparticles whose finite dimensions are relevant to the analysis of radiative heat transfer. Typically, in such cases, it is incumbent to exploit general-purpose techniques to compute field response quantities entering $\mathcal{T}(\omega)$, for the geometry in question, in terms of the system's Green's function. One such powerful general scattering formalism was developed by Krüger and coworkers (Bimonte et al., 2017, Krüger et al., 2012), arriving at the general formula (for reciprocal media),

$$
\mathcal{T}(\omega)=4 \operatorname{Tr}\left[\mathbb{R}_{2}^{*} \mathbb{W}_{1,2} \mathbb{R}_{1} \mathbb{W}_{2,1}^{*}\right]
$$

in terms of the radiation operator $\mathbb{R}_{p}=\mathbb{G}_{0}\left(\operatorname{Im}\left(\mathbb{T}_{p}\right)\right.$ $\left.\mathbb{T}_{p} \operatorname{Im}\left(\mathbb{G}_{0}\right) \mathbb{T}_{p}^{*}\right) \mathbb{G}_{0}^{*}$ and scattering operator $\mathbb{W}_{p q}=\mathbb{G}_{0}^{-1}(\mathbb{1}-$ $\left.\mathbb{G}_{0} \mathbb{T}_{p} \mathbb{G}_{0} \mathbb{T}_{q}\right)^{-1}$ for bodies $p, q \in\{1,2\}$ defined in terms of the scattering T-operators $\mathbb{T}_{p}$, which depend on the material properties and shape of the bodies and the Green's function operator $\mathbb{G}_{0}$ in vacuum. The strength of this formulation lies in its broad applicability, as it generalizes beyond systems with discrete or continuous translational symmetry: it can in principle be used for arbitrary geometries, including compact bodies whose finite sizes in each dimension are relevant, with faster numerical convergence for appropriate choices of basis functions. Additionally, while this T-operator formalism casts thermal radiation in terms of volumetric scattering quantities, related contemporaneous surface integral equation formulations (Rodriguez et al., 2013a) can similarly recover known semi-analytical results for uniform planar media and be computationally amenable to general compact or extended geometries by casting thermal radiation purely in terms of surface unknowns, vastly reducing the computational complexity of calculations.

Furthermore, beyond simply aiding in generalizations of computations beyond extended media with translational symmetry, the T-operator formalism can shed further light on the number of contributing transmission channels to $\mathcal{T}(\omega)$. In the operators of Eq. (16), an operator of particular interest (Miller, 2000, 2007, Miller et al. 2015, Molesky et al., 2020: Venkataram et al., 2020) is the off-diagonal block $\mathbb{G}_{0(2,1)}$ of the Green's function connecting points $\mathbf{r}^{\prime}$ restricted to the volume of body 1 and $\mathbf{r}$ restricted to the volume of body 2. At first glance, the ability of electromagnetic fields to propagate through vacuum, or equivalently the coupling of all pairs of volumetric degrees of freedom in each of the different bodies, suggests that the number of channels will scale like the volume of each body. However, the electromagnetic surface equivalence theorem (Harrington, 1989. Otey et al., 2014; Reid and Johnson, 2015; Reid et al. 2013b; Rengarajan and Rahmat-Samii, 2000; Rodriguez 


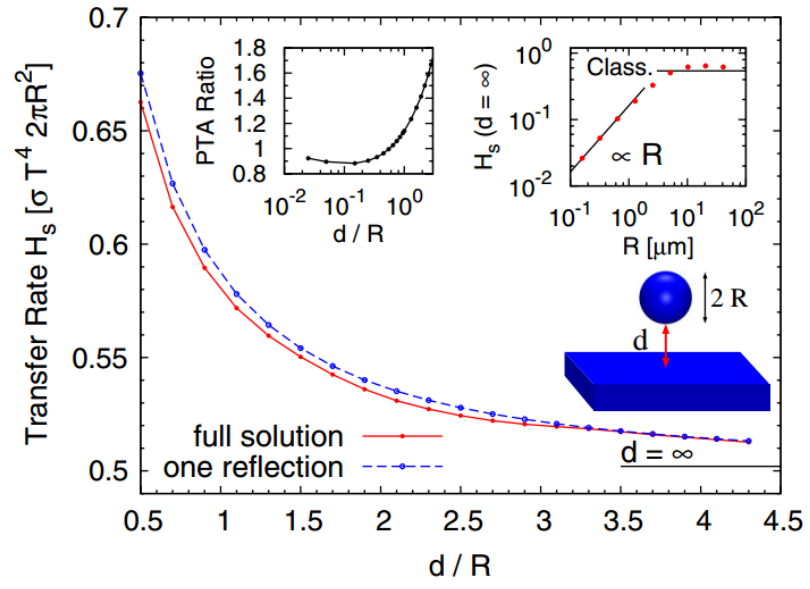

FIG. 9 (Krüger et al., 2011) Transferred power $H_{s}$ by NFRHT between a $\overline{\mathrm{SiO}_{2}}$ sphere with radius $R=5 \mu \mathrm{m}$ at $300 \mathrm{~K}$ and $\mathrm{a} \mathrm{SiO}_{2}$ plane at $0 \mathrm{~K}$ as a function of distance $d$. The transferred power is normalized to the power emitted by a black body with a surface area given by the cross section of the sphere. From (Krüger et al. 2011).

et al. 2013a) shows that the electromagnetic fields radiated by any volumetric polarization distribution to the exterior of some fictitious bounding surface can be exactly reproduced in that exterior region by an equivalent surface current distribution, therefore suggesting that the rank of $\mathbb{G}_{0(2,1)}$ actually scales with the surface area of each body; as shown by Polimeridis et al. (Polimeridis et al. 2015), it is indeed the effective rank of this offdiagonal scattering operator that determines the number of contributing transmission channels $\tau_{n}$.

Based on the scattering approach and standard Green's function formalism, there have been many studies of the heat flux between a sphere and a plane, as shown in Fig. 9 and between two spheres (Krüger et al. 2011, Narayanaswamy et al., 2008, Otey and Fan, 2011a, Sasihithlu and Narayanaswamy, 2011). Reviews highlighting other studies of NFRHT in non-planar geometries can be found in (Bimonte et al. 2017, Otey et al., 2014). Early studies of heat transfer between compact bodies typically focused on high-symmetry objects with simple shape. However, there have been far fewer studies of NFRHT in nanostructured compact bodies compared to the preponderance of examples for extended media (including the previously-discussed gratings, photonic crystals, and metasurfaces) because the former, unlike the latter, does not easily succumb to semianalytical expressions for arbitrary geometries in the absence of symmetries like continuous or discrete translational invariance. With this in mind, the next section discusses the development of various numerical methods to compute radiative heat transfer in a broad array of systems.

\section{F. Numerical methods}

Advances in computational hardware and numerical algorithms have led to an explosion of computational methods to study radiative heat transfer. Notably, the facts that the Landauer form of the radiative heat transfer power depends only on the Bose function $n(\omega, T)$ and the Landauer energy transmission spectrum $\mathcal{T}(\omega)$, and that the latter in Eq. (16) only depends on classical electromagnetic scattering quantities, means that standard computational techniques may be readily applied to studying radiative heat transfer. These methods, illustrated schematically with examples in Fig. 10. essentially fall into one of two categories, depending on the choice of either a spectral or localized basis expansion (Bimonte et al., 2017, Cuevas and García-Vidal, 2018, Otey et al., 2014 Reid et al. 2013a; Song et al. 2015a), each of which brings a set of benefits and drawbacks.

\section{Spectral methods}

Techniques based on spectral expansions (Bimonte et al. 2017; Krüger et al. 2012) express the T-operators of each individual body in terms of delocalized spectral functions (e.g. the spherical vector waves discussed above). These basis functions include but are not limited to plane waves (Fourier basis) (Bimonte, 2009, Jin et al., 2017a, Messina and Antezza, 2011b Messina and Ben-Abdallah, 2013; Messina et al., 2016b), Bloch waves (Ben-Abdallah et al., 2010, Francoeur et al. 2009. Messina et al. 2017b; Narayanaswamy and Chen, 2005. Tschikin et al., 2012a), and spherical or cylindrical harmonics (Krüger et al., 2011, McCauley et al., 2012, Narayanaswamy et al., 2008; Otey and Fan, 2011a). The use of these basis functions is most convenient when the geometries involved exhibit discrete or continuous symmetries, like translation or rotation, as that can make the resulting matrix expressions for the relevant operators nearly diagonal, making computations far more efficient. However, in the absence of such symmetries, or when different bodies have shapes of different symmetries, not only are the resulting matrices dense, but the convergence with respect to increasing numbers of basis functions slows dramatically. Furthermore, we note that with few exceptions, such as work on graphene sheets (Ilic et al. 2012a; Neto et al., 2009; Sernelius, 2012; Wunsch et al. 2006), most applications of these spectral techniques have in practice focused on simple local isotropic homogeneous susceptibilities $\chi(\omega)$.

\section{Decomposition methods}

By contrast, techniques based on localized expansions (Cuevas and García-Vidal, 2018, Otey et al., 2014, Song et al., 2015a) express either T-operators or Maxwell Green's functions in terms of localized basis functions. 


\section{Spectral methods}
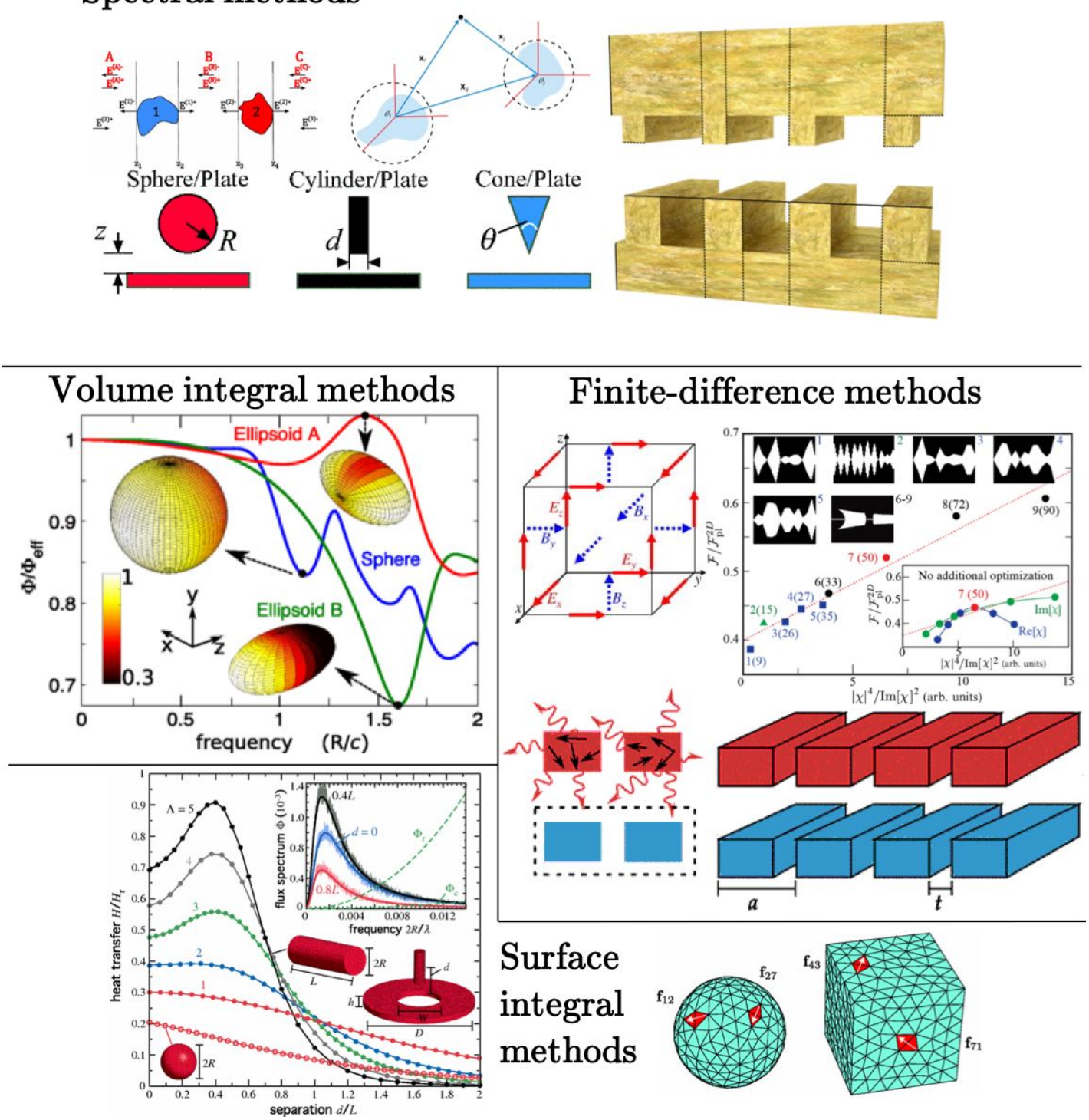

FIG. 10 Collage of selected computational methods. Schematics of basis functions, along with selected results, for spectral (Bimonte et al., 2017, Krüger et al., 2012, McCauley et al. 2012, Messina and Antezza, 2011b, Messina et al., 2017b), finite-difference (Jin et al., 2019, Rodriguez et al., 2011; Werner et al., 2013), volume integral (Polimeridis et al., 2015), and surface integral (Reid et al., 2013b; Rodriguez et al., 2013a b) methods.

One such technique is the finite-difference frequency domain method (Jin et al., 2019, Wen, 2010), in which Maxwell's equations in the frequency domain are discretized on a lattice of grid points. In the context of RHT, fields in response to individual dipolar sources embedded in the radiating objects can be computed independently and then summed according to weights determined by the fluctuation-dissipation theorem; alternatively, the uncorrelated nature of dipolar sources at different spatial positions means that all such fluctuating sources can be simultaneously introduced and modelled as stochastic, random sources with correlation functions given by the fluctuation-dissipation theorem (requiring ensemble averages over many source realizations to reduce noise, as in
Monte-Carlo integration). The latter interpretation lends itself to a direct Langevin or stochastic time-domain simulation of Maxwell's equations (Rodriguez et al., 2011). This last class of time-domain method has the added benefit that discretized spatial differential operators are represented as sparse matrices, and allows representations of broad classes of nonlocal (spatially dispersive) susceptibility models in terms of spatial differential operators, such as the hydrodynamic model (Klimchitskaya and Mostepanenko, 2015, Xiao et al., 2016), all the while being applicable to arbitrary body shapes. On the other hand, multiscale or large problems become particularly challenging to simulate as the propagation of electromagnetic fields through vacuum means that the entire space 
between bodies must also be discretized, even if the separation is much larger than relevant body feature sizes, so the resulting convergence with respect to resolution can be prohibitively slow.

A related class of technique is the so-called volumeintegral formulation of Maxwell's equations (Jin et al. 2017b, 2016, Polimeridis et al. 2015), of which the discrete dipole approximation (DDA) (Edalatpour et al. 2016, Edalatpour and Francoeur, 2014, 2016; Ekeroth et al. 2017) may be thought of as a special case. In general, volume integral formulations use various classes of localized basis functions as basis expansions for $\mathrm{T}$ operators and $\mathbb{G}_{0}$. Unlike finite-difference methods, these techniques have the advantage of only requiring basis functions within the volumes of material bodies, with the full scattering problem represented by expressing the full Green's function in terms of the individual materials' scattering matrices and the analytically known freespace Green's function of the corresponding intervening medium. As expected, however, different choices of basis functions offer challenges and tradeoffs with respect to numerical convergence. As further elucidated below, DDA is effectively a volume integral formulation in which each body is discretized into point dipolar particles with equivalent Clausius-Mossotti polarizabilities: this approximation typically yields accurate results for dielectric media, but suffers from poor convergence when simulating metals with highly delocalized plasmons. In contrast, volume-integral formulations guaranteed to converge require a so-called Galerkin discretization of the problem based on use of either voxel (Polimeridis et al., 2015) or Schaubert-Wilton-Glisson (tetrahedral) (Reid et al., 2017) basis functions. In either case, the basis functions may be identical and displaced on a regular grid/lattice covering each body, in which case the matrix representation of $\mathbb{G}_{0}$ may be sparse (and therefore computationally easier to handle) due to the translational symmetries inherent in $\mathbb{G}_{0}$, though this often comes at the costs of computing matrix elements of $\mathbb{G}_{0}$ for regions where no materials are present, or of losing flexibility over discretizing certain regions more finely than others (Polimeridis et al. 2015). Exactly the opposite tradeoff occurs if the volumes are discretized in an irregular manner, with different weights given to different basis functions (Reid et al. 2017): it then becomes possible to discretize certain regions more finely than others, which is of particular relevance to near-field radiative heat transfer between large bodies where only a few fine features are very close to one another, but at the cost of the matrix representation of $\mathbb{G}_{0}$ becoming dense due to the loss of obvious symmetries in the representation. Furthermore, in all cases, volume integral formulations can model inhomogeneous and anisotropic susceptibilities and even temperature gradients (Jin et al., 2016, Polimeridis et al., 2015), but modeling nonlocal susceptibilities has proved to be more of a challenge.

A class of techniques related to the volume integral formulation are those based on the surface integral formula- tion (Rodriguez et al. 2013a b) of Maxwell's equations. These techniques compute the Landauer energy transmission spectrum $\mathcal{T}$ according to a formula that looks superficially similar to Eq. (16) but whose derivation and implementation requires a different set of techniques. In particular, surface-integral formulations make consistent use of the surface equivalence theorem (Harrington, 1989 , Otey et al., 2014; Reid and Johnson, 2015; Reid et al. 2013b; Rengarajan and Rahmat-Samii, 2000; Rodriguez et al. 2013a) to recast all free polarization sources and total electromagnetic fields in terms of equivalent surface currents, with the relevant operators being the Green's functions of the homogeneous susceptibilities comprising each body, as well as the surface integral operator relating incident fields to induced equivalent surface currents. In principle, the operators relevant to the surface integral formulation can be expanded in a spectral basis (Rodriguez et al. 2013a), but as in the T-operator formulation, convergence suffers for bodies that do not exhibit requisite symmetries. Instead, it is more common to expand the relevant operators in a localized basis like the Rao-Wilton-Glisson basis (Rodriguez et al., 2013a b) of tetrahedral functions.

Finally, we point out that any of these frequency domain methods could have instead been cast in the time domain. In the context of computational electromagnetism, this is most commonly achieved by using the finite-difference time domain method (Luo et al., 2004; Rodriguez et al. 2011). This has many of the same benefits and detriments of the aforementioned finitedifference frequency domain method. Techniques based on molecular dynamics have also been used to compute radiative heat transfer in systems comprising nanoparticles (Domingues et al. 2005), though the scaling of the volume with the cube of the number of atoms makes computations unwieldy in practice for large nanoparticles. For both of these time domain techniques, the main advantages are their generality with respect to materials, the simple computational implementation (as the temporal evolution operators are represented as sparse matrices), the ability to extract dynamical information, and their ability in principle to incorporate nonlinear material response. In the case of molecular dynamics, susceptibilities can be simulated fairly generally as the method is based on simulating classical Newtonian particle dynamics, though interactions other than harmonic or Coulomb couplings are typically based on empirical rather than ab-initio models. The main disadvantages for both sets of techniques are losses in computational efficiency from needing to explicitly simulate fluctuating polarization sources obeying fluctuation-dissipation statistics, which requires that averages be taken over a large ensemble of calculations. 


\section{G. Upper bounds on near-field heat transfer}

As noted above, the Stefan-Boltzmann formula or blackbody limit was derived over a century ago under the assumptions of ray optics, and consequently fails to provide an upper bound of the maximum heat flux that can be extracted from an object in the near-field regime. While it is known that, as in far-field emission, appropiate choice of object geometry (nanostructuring) and materials can enhance NFRHT, the lack of such a limit applicable in the near field begs the question: how much more room for improvement can be expected from either of these design criteria? Over the past few decades, there have been several succesful attempts at addresing this fundamental question, starting with analyses of maximum NFRHT achievable in planar geometries (where the main design criterion is the choice of material) (BenAbdallah and Joulain, 2010, Biehs et al., 2012, Volokitin and Persson, 2004) and followed more recently by limits applicable to arbitrary nanostructures and materials (Miller et al., 2015, Venkataram et al., 2020). Technically speaking, it is clear that upper limits to the heat flux are determined by bounds on the transmission coefficient $\mathcal{T}(\omega)$ per unit area in Eq. (5), which is itself determined by the per-channel transmission factors $\tau_{n}(\omega)$ entering Eq. (6). The aim of arriving at a bound on RHT is therefore to discern the maximum number and contribution of tranmission channels that may be excited by a yet unknown optimal choice of material and geometry.

In the case of two planar bodies, the maximum heat flux is determined by the bounds on the transmission coefficient $\mathcal{T}(\omega)$ per unit area in Eq. (7), which is determined by the transmission factor $\tau_{\alpha}(\omega, \kappa) \in[0,1]$ corresponding to transversal waves of frequency $\omega$, lateral wavevector $\kappa$, and polarization $\alpha=s, p$. It is then clear that $\mathcal{T}(\omega)$ can be maximized if the transmission factor $\tau_{\alpha}(\omega, \kappa)$ is maximal over a broad frequency and lateral wavevector range. For example, when assuming that at a given frequency, all transversal waves contribute a maximal transmission factor of unity up to some threshold value $\kappa_{\max }$, the upper bound for the transmission coefficient per unit area between two planar bodies can be written as

$$
\mathcal{T}_{\mathrm{pl}}(\omega) \leq 2 \int_{0}^{\kappa_{\max }} \frac{\mathrm{d} \kappa}{2 \pi} \kappa=N(\omega),
$$

where $N(\omega)$ may be interpreted as the number of contributing transmission modes or channels per unit area (Ben-Abdallah and Joulain, 2010, Biehs and Greffet, 2010a). By definition, the contribution of propagating waves is restricted to $\kappa<k_{0}$. Hence, setting $\kappa_{\max }=k_{0}$, one obtains the maximum value of $\mathcal{T}(\omega)=k_{0}^{2} / 2 \pi$ for propagating waves. Inserting this maximum value in Eq. (7), one finds that the largest heat flux $\Phi_{\mathrm{pr}}^{\max }$ that can ever be carried by propagating waves is precisely the black-body value $\Phi_{\mathrm{BB}}$ given by Stefan-Boltzmann's law (Bergman et al., 2011, Planck, 1914). Thus, it is the additional contribution coming from evanescent waves with $\kappa \geq k_{0}$ and not accounted for in Stefan-Boltzmann's law that allows NFRHT to surpass the blackbody limit.

At first glance, it may appear that there is no upper bound to $\kappa_{\max }$ in the evanescent sector, at least within the scope of local continuum electromagnetism, suggesting that $\mathcal{T}_{\mathrm{pl}}(\omega)$ is unbounded. However, even simple considerations imply otherwise. For instance, inside a dielectric, the largest possible lateral wavevector allowed is given by the edge of the Brillouin zone $\pi / a$, where $a$ is the lattice constant of the medium. Hence, only waves up to wavevectors $\kappa_{\max } \approx \pi / a$ contribute heat flux. Ignoring possible band degeneracies and physical constraints imposed by material and geometric considerations, this gives the following idealized upper bound on the maximum possible heat flux between two dielectrics (Volokitin and Persson, 2004):

$$
\Phi_{\mathrm{pl}, \text { ideal }}^{\max } \approx \frac{k_{\mathrm{B}}^{2} \pi^{2}}{24 \hbar a^{2}}\left(T_{1}^{2}-T_{2}^{2}\right) .
$$

Assuming a wavevector cutoff set by a lattice constant on the order of the atomic scale $\left(a \approx 10^{-10} \mathrm{~m}\right)$, and roomtemperature operation $\left(T_{1}=300 \mathrm{~K}\right.$ and $\left.T_{2}=0 \mathrm{~K}\right)$, yields a heat flux of $10^{13} \mathrm{Wm}^{-2}$ that is unrealistically large compared to the black-body value of about $460 \mathrm{Wm}^{-2}$. Taking into account the nature of evanescent waves within the vacuum gap between the two planar materials, one may derive a more sensible upper bound. For instance, the field amplitude of evanescent waves of a given $\kappa$ in the quasi-static regime drops exponentially as $\exp (-\kappa z)$ with respect to the distance $z$ from the interface. As a consequence, one can expect that only evanescent waves having $1 / \kappa \approx z>d$ or $\kappa<1 / d$ can meaningfully contribute to the heat flux between two planar interfaces a distance $d$ apart, suggesting that $\kappa_{\max } \approx 1 / d$. In (Ben-Abdallah and Joulain 2010), it is argued that only evanescent modes with $1 / \kappa \approx z>d / 2$ overlap significantly and contribute, so a distance-dependent cutoff $\kappa_{\max } \approx 2 / d$ is used to provide an estimate of the upper limit for $\mathcal{T} \leq 2 / \pi d^{2}$, leading to the following gap-dependent upper bound on the net heat flux (Ben-Abdallah and Joulain, 2010):

$$
\Phi_{\mathrm{pl}, \mathrm{gap}}^{\max }=\frac{k_{\mathrm{B}}^{2}}{6 \hbar d^{2}}\left(T_{1}^{2}-T_{2}^{2}\right) .
$$

The choice of $\kappa_{\max }=1 / d$ would decrease this estimate by a factor of $1 / 4$. Note that this cutoff is consistent with the fact that $\mathcal{T}$ scales as $\exp (-2 \kappa d)$ with the separation distance $d$. A similar simple and general, albeit material independent expression for the upper limit of the heat flux contribution has also be found for the case of two hyperbolic metamaterials (Biehs et al. 2012 ).

Material considerations further constrain the allowed heat flux between planar media. In particular, Biehs and Greffet (Biehs and Greffet, 2010a) derived a more realistic frequency-dependent cutoff $\kappa_{\max }=\ln [2 / \operatorname{Im}(\chi)] / d$ that accounts for the impact of material absorption through the material-specific loss rate $\operatorname{Im}[\chi(\omega)]$, where 
$\chi$ is the medium's susceptibility. In particular, knowledge of the analytical form of the reflection coefficients at an interface can be used to show that the maximum flux occurs for materials satisfying the surface-mode resonance condition, $\operatorname{Re}(1 / \chi)=-1 / 2$. The fact that in the quasi-static regime the heat flux scales like $1 / d^{2}$ can be understood from the fact that the number of contributing evanescent modes per unit area scales like $1 / d^{2}$ (BenAbdallah and Joulain, 2010, Biehs and Greffet, 2010a; Biehs et al. 2012). Generalizations of related analysis to bound the performance of planar metasurfaces (nanstructured materials with subwavelength systems) have recently been made (Biehs et al., 2012: Miller et al., 2014), showing for instance that metasurfaces cannot significantly enhance NFRHT beyond planar thin films.

Efforts aimed at identifying the number and relative contribution of transmission channels that may arise in non-planar media require a different framework. Recently, Miller et al (Miller et al., 2015) recast radiative heat transfer between two bodies as a series of independent absorption and emission problems (ignoring additional constraints posed by the presence of multiple scattering among the two objects) to obtain bounds that only depend on the bodies' material susceptibilities and separation. In particular, recent work showed that given an incident field on an object of susceptibility $\chi(\omega)$, the maximum polarization field that can arise at any point inside the object at a frequncy $\omega$ depends on the "material response factor" (Miller et al. 2016),

$$
\zeta(\omega)=\frac{|\chi(\omega)|^{2}}{\operatorname{Im}[\chi(\omega)]}
$$

Such a figure of merit yields a measure of the resistivity or dissipation of the medium and thereby captures the impact of losses on the resonant optical response of a body. The material response factor arises from the optimal magnitude of the T-operator for maximal absorption in isolation (Miller et al., 2016), and encodes electromagnetic many-body and multiple scattering effects within the body in isolation; this optimal magnitude is achievable at a polaritonic resonance, determined by the value of $\operatorname{Re}(1 / \chi)$, which in turn can be tailored through nanostructuring. Exploiting the maximum polarization responsivity of a medium in combination with electromagnetic reciprocity, Miller et al found an upper bound on the net transmission $\mathcal{T} \leq 4 \zeta_{1} \zeta_{2} \int_{V_{1}} \mathrm{~d} \mathbf{r}^{\prime} \int_{V_{2}} \mathrm{~d} \mathbf{r} \sum_{i, j}\left|G_{0}\left(\omega, \mathbf{r}, \mathbf{r}^{\prime}\right)\right|^{2}$ that depends quadratically on the effective loss rate of the system $\zeta=\sqrt{\zeta_{1} \zeta_{2}}$, with $\zeta_{1}$ and $\zeta_{2}$ denoting the material factors of the bodies, and on the integral of the vacuum Green's function over volumes $V_{1}$ and $V_{2}$ representing any convenient domain that may contain bodies 1 and 2 , respectively. Such a double integral may be cast as a Frobenius norm of the off-diagonal matrix $\mathbb{G}_{0(2,1)}$, which was previously identified in related works by D. A. Miller et al (Miller, 2000, 2007) on optical communication limits. However, such an analysis depends crucially on the assumption that each body is capable of simultaneously and optimally emitting electromagnetic fields in the absence of the other, and of optimally absorbing electromagnetic fields in the presence of the other, which effectively neglects additional physical constraints arising from the unavoidable impact of multiple scattering between the two bodies. As a result, the limits have been shown to be tight in situations where multiple scattering can be neglected, namely quasistatic media subject to relatively large material losses (Jin et al., 2019). This problem becomes particularly acute in the context of bounds on extended structures, where the inability to account for tighter bounds on the transmission eigenvalues causes the quadratic dependence on $\zeta$ to far outstrip the observed logarithmic dependence on $\zeta$ seen in polaritonic planar media near the resonance condition $\operatorname{Re}(1 / \chi)=-1 / 2$ (and predicted by the above planar bounds), suggesting more room for enhancements in NFRHT through nanostructuring than has been observed in practice.

In recent work, Venkataram et al (Molesky et al. 2020 , Venkataram et al., 2020) developed a set of algebraic techniques to derive tighter bounds on NFRHT that incorporate not only constraints on material response but also multiple scattering. Specifically, the transmission coefficient for two arbitrarily shaped bodies at any given frequency $\omega$ was found to be bounded above by,

$$
\begin{aligned}
\mathcal{T}_{\operatorname{arb}}(\omega) & =\sum_{n} \tau_{n}(\omega) \\
& \leq \sum_{n} \begin{cases}1, & \zeta_{1} \zeta_{2} g_{n}^{2} \geq 1 \\
\frac{4 \zeta_{1} \zeta_{2} g_{n}^{2}}{\left(1+\zeta_{1} \zeta_{2} g_{n}^{2}\right)^{2}}, & \zeta_{1} \zeta_{2} g_{n}^{2}<1\end{cases}
\end{aligned}
$$

where the dependence on $\omega$ inside the various factors has been deprecated. These bounds depend not only on the resistivity $\zeta_{i}(\omega)$ of each body $i=\{1,2\}$ at the given frequency, but also on a set of "radiative efficacy" coefficients $g_{n}(\omega)$ denoting the singular values of the vacuum off-diagonal Maxwell Green's function $\mathbb{G}_{0(2,1)}$ connecting dipoles in one object to the resulting fields on the other. Moreover, the bounds move beyond simply identifying the set of channels able to contribute to heat transfer, previously estimated on the basis of the effective rank of $\mathbb{G}_{0(2,1)}$, and instead exploit the specific singular values of $\mathbb{G}_{0(2,1)}$ in combination with the loss rate of the medium to quantitatively determine the maximum possible transmission for each channel. Once the set of channels that could possibly contribute (having nonzero radiative coupling $\left.g_{n}\right)$ is identified, the ability of each transmission channel to saturate the Landauer upper bound of unity $\left(\tau_{n} \leq 1\right)$ is determined by the degree to which the radiative efficacies are able to overcome material losses, captured by the condition $\zeta_{1} \zeta_{2} g_{n}^{2} \geq 1$; the per-channel bound is less than unity for those channels unable to meet such a condition. In addition to correctly reproducing the transition and eventual saturation in the growth of NFRHT between dipolar nanoparticles, from materialloss-dominated growth in the polarization response to the Landauer tranmission bounds of unity, these limits reveal that extended nanostructured bodies cannot sig- 
nificantly outperform resonant planar polaritonic slabs even in principle. Specifically, evaluation of the radiative efficacies for any set of nanostructures contained within semi-infinite half-space domains yields a limit on the net transmission of

$$
\begin{aligned}
\mathcal{T}_{\text {arb }}(\omega) \times d^{2} / A \leq \\
\frac{1}{2 \pi} \begin{cases}\ln \left(1+\frac{\zeta_{1} \zeta_{2}}{4}\right), & \zeta_{1} \zeta_{2}<4 \\
\frac{1}{2} \ln \left(\zeta_{1} \zeta_{2}\right)+\frac{1}{8}\left[\ln \left(\frac{\zeta_{1} \zeta_{2}}{4}\right)\right]^{2}, & \zeta_{1} \zeta_{2} \geq 4\end{cases}
\end{aligned}
$$

which exhibits a weak squared-logarithmic dependence on $\zeta$, in line with the observed logarithmic peak value of $\mathcal{T}$ for planar slabs at a polaritonic resonance (Biehs and Greffet, 2010a; Miller et al. 2015).

Based on this recent analysis, it is evident that the observed inability of nanostructuring to significantly enhance the amplitude of $\mathcal{T}$ at any given frequency beyond what is achievable with resonant planar materials is a "feature" of the underlying physics of NFRHT, and not a "bug" in sampling a limited design space: the maximum channel able to saturate the Landauer transmission limit of unity for any nanostructure scales logarithmically as $\frac{1}{2 d} \ln \left(\frac{\zeta_{1} \zeta_{2}}{4}\right)$ provided the system is in the underdamped (resonant) regime $\zeta_{1} \zeta_{2} \geq 4$. Intuitively, this result may be seen as dissonant with the established utility of nanostructuring for enhancing far-field electromagnetic absorption and scattering, and the significantly stronger enhancements of local densities of states that can arise in the vicinity of structured materials. However, the channels of radiative heat transfer between two separable bodies in proximity have little to do with the channels that carry energy away from a body (or an aggregate two-body system), so there is no reason to believe that enhancement of the latter transmission channel contributions would necessarily increase the former.

The transition from a quadratic (Miller et al., 2015) to a much weaker logarithmic (Venkataram et al., 2020) dependence of the bounds on material conductivity once multiple-scattering constraints are introduced illustrates the restricted and prohibitive nature of nanostructuring in tailoring mutual scattering across a wide range of resonant channels. Such a tradeoff precisely explains why the success of nanostructuring in enhancing local fields does not readily translate into equivalent enhancements in NFRHT. As reviewed in Sec.II.B and Sec. II.D, metallic nanostructures can indeed greatly enhance heat exchange compared to their planar counterparts, but as these limits suggest, not much more than what may be achieved with planar polar dielectrics. Finally, while multiple scattering ultimately hampers the maximum heat exchange that any two bodies can experience, as we shall see in the next section, it underlies several important transport effects in many-body systems.

\section{MANY-BODY SYSTEMS}

Until this last decade, theoretical and experimental work in the topic near-field radiative heat transport was primarily relegated to the study of heat exchange between two objects, while transport in systems composed of objects in mutual interactions remained largely unexplored and out of the reach of classical FE. In 2011, Ben Abdallah et al. (Ben-Abdallah et al. 2011) laid out the theoretical foundations for studying NFRHT in simple manybody systems made of small interacting objects in the dilute regime, paving the way for a new research direction on the topic of nanoscale heat transfer. Since then, numerous works have revealed new many-body effects, including the emergence of new physical and transport behaviors, and unraveling a large number of potential applications in domains such as nanoscale thermal management, energy-conversion technology, and information processing. In the following sections, we describe these peculiarities.

\section{A. Heat flux in dipolar many-body systems}

Understanding the mechanisms that drive light matter interactions is one of the main goal in optics. In the following, we address the problem of light absorption and thermal emission by a set of small objects in which cooperative interactions as well as heat exchange take place in these systems.

\section{Light absorption in dipolar systems}

To start let us consider the case of non-emitting objects which are only able to scatter and absorb light from an external source, i.e. we are neglecting thermal radiation at this stage. In the simplest case of a small isolated particle located at position $\mathbf{r}^{\prime}$ in vacuum, the optical response of this particle can be described by the response to a simple permanent dipolar electric moment $\mathbf{p}\left(\mathbf{r}^{\prime}\right)$.

The electric field produced at point $\mathbf{r}$ around this dipole takes the following form

$$
\mathbf{E}_{\mathrm{p}}(\mathbf{r})=\omega^{2} \mu_{0} \mathbb{G}_{0}\left(\mathbf{r}, \mathbf{r}^{\prime}\right) \mathbf{p}\left(\mathbf{r}^{\prime}\right)
$$

Here (Novotny and Hecht, 2006)

$$
\begin{array}{r}
\mathbb{G}_{0}\left(\mathbf{r}, \mathbf{r}^{\prime}\right)=\frac{\exp \left(\mathrm{i} k_{0} \rho\right)}{4 \pi \rho}\left[\left(1+\frac{\mathrm{i} k_{0} \rho-1}{k_{0}^{2} \rho^{2}}\right) \mathbb{1}\right. \\
\left.+\frac{3-3 i k_{0} \rho-k_{0}^{2} \rho^{2}}{k_{0}^{2} \rho^{2}} \widehat{\boldsymbol{\rho}} \otimes \widehat{\boldsymbol{\rho}}\right]
\end{array}
$$

is the free space Green tensor defined with the unit vector $\widehat{\boldsymbol{\rho}} \equiv \boldsymbol{\rho} / \rho, \boldsymbol{\rho}=\mathbf{r}-\mathbf{r}^{\prime}, k_{0}=\omega / c$ is the wave vector while $\mathbb{1}$ denotes the unit dyadic tensor and $\mu_{0}$ denotes the vacuum permeability. When this particle is illuminated by an incident field $\mathbf{E}_{\text {inc }}$, the local electric field $\mathbf{E}_{\text {loc }}$ measured at any point $\mathbf{r}$ is the superposition of the 
incident field and the field generated (scattered) by the dipole. Therefore, according to expression (23), this field decomposes into

$$
\mathbf{E}_{\mathrm{loc}}(\mathbf{r})=\mathbf{E}_{\mathrm{inc}}(\mathbf{r})+\omega^{2} \mu_{0} \mathbb{G}_{0}\left(\mathbf{r}, \mathbf{r}^{\prime}\right) \mathbf{p}\left(\mathbf{r}^{\prime}\right) .
$$

The electromagnetic power $\mathcal{P}$ dissipated in the particle can be calculated from the rate of work

$$
\mathcal{P}_{\text {abs }}=\frac{1}{2} \int_{V} \mathrm{~d} V \operatorname{Re}\left(\mathbf{j}^{*} \cdot \mathbf{E}_{\mathrm{loc}}\right)
$$

done by the electromagnetic field in a volume $V$ including the particle. Here $\mathbf{j}$ denotes the local electric current density in the volume $V$. In the dipolar approximation $\mathbf{j}\left(\mathbf{r}^{\prime}\right)=-\mathrm{i} \omega \mathbf{p} \delta\left(\mathbf{r}-\mathbf{r}^{\prime}\right)$ so that

$$
\mathcal{P}_{\mathrm{abs}}=\frac{1}{2} \operatorname{Re}\left(\mathrm{i} \omega \mathbf{p}^{*} \cdot \mathbf{E}_{\mathrm{loc}}\right)=-\frac{\omega}{2} \operatorname{Im}\left(\mathbf{p}^{*} \cdot \mathbf{E}_{\mathrm{loc}}\right) .
$$

Using the following relation

$$
\mathbf{p}\left(\mathbf{r}^{\prime}\right)=\epsilon_{0} \alpha \mathbf{E}_{\text {inc }}\left(\mathbf{r}^{\prime}\right)
$$

between the incident field and the dipolar moment, where $\alpha$ is the electric polarizability, the power dissipated in the particle reads (Tretyakov, 2014)

$$
\mathcal{P}_{\text {abs }}=\frac{\omega\left|\mathbf{E}_{\mathrm{inc}}\right|^{2} \epsilon_{0}}{2}\left(\operatorname{Im}[\alpha]-\frac{k_{0}}{6 \pi}|\alpha|^{2}\right) .
$$

It is common to quantify light absorption using the absorption cross-section defined as the ratio

$$
\sigma_{\mathrm{abs}}=\frac{\mathcal{P}_{\mathrm{abs}}}{\mathcal{F}_{\mathrm{inc}}}
$$

of this dissipated power by the incident flux

$$
\mathcal{F}_{\text {inc }}=\frac{c \epsilon_{0}}{2}\left|\mathbf{E}_{\text {inc }}\right|^{2} .
$$

For a collection of dipoles located at the position $\mathbf{r}_{i}$ $(i=1, \ldots, N)$ the multi-scattering process between the particles must be taken into account (Langlais et al. 2014). Under an external illumination by an incident field $\mathbf{E}_{\text {inc }}$, the local electric field $\mathbf{E}_{\text {loc }}$ measured at any point results from the superposition of the incident and all scattered fields as

$$
\mathbf{E}_{\mathrm{loc}}(\mathbf{r})=\mathbf{E}_{\mathrm{inc}}(\mathbf{r})+\omega^{2} \mu_{0} \sum_{j=1}^{N} \mathbb{G}_{0}\left(\mathbf{r}, \mathbf{r}_{j}\right) \mathbf{p}_{j} .
$$

By introducing the notation $\mathbf{p}_{i}=\mathbf{p}\left(\mathbf{r}_{i}\right), \mathbf{E}_{\mathrm{loc}, i}=\mathbf{E}_{\mathrm{loc}}\left(\mathbf{r}_{i}\right)$, $\mathbf{E}_{\text {inc }, i}=\mathbf{E}_{\text {inc }}\left(\mathbf{r}_{i}\right)$ the total power absorbed by this set of dipoles takes the general form (Hugonin et al. 2015 )

$$
\begin{aligned}
\mathcal{P}_{\mathrm{abs}}=\frac{\omega}{2} & \left(\sum_{i=1}^{N} \operatorname{Im}\left(\mathbf{p}_{i} \cdot \mathbf{E}_{\text {inc }, \mathrm{i}}{ }^{*}\right)\right. \\
& \left.-\sum_{i, j=1}^{N} \operatorname{Im}\left(\mathbf{p}^{*}{ }_{i} \boldsymbol{D}_{i j} \mathbf{p}_{j}\right)\right)
\end{aligned}
$$
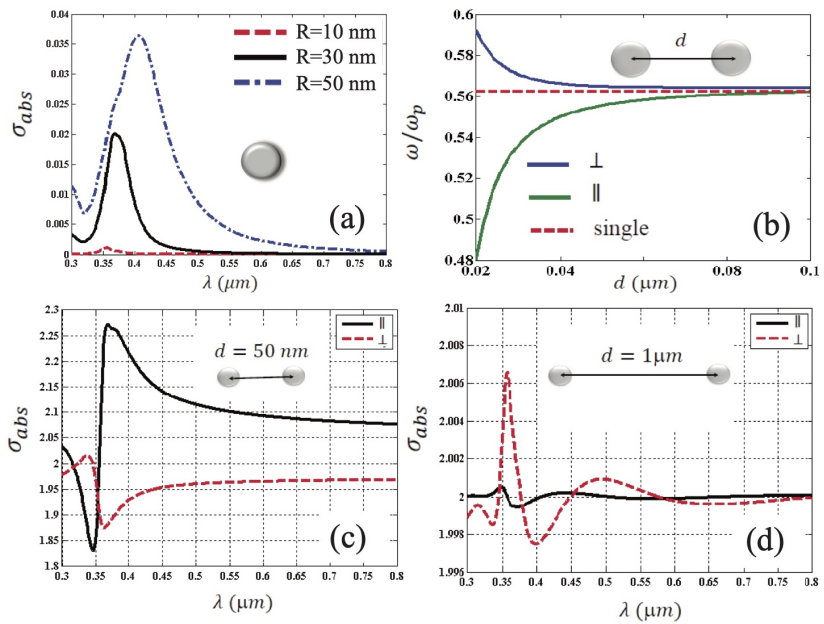

FIG. 11 (a) Absorption cross-section of spherical silver nanoparticles with respect to the wavelength. (b) Orthogonal and parallel configurational resonance frequencies for a dimer of silver nanoparticles $(R=10 \mathrm{~nm})$ in vacuum with respect to their separation distance $d$. The red horizontal line represents the plasmon resonance of an isolated particle.(c)-(d) Absorption cross-sections for a dimer of silver nanoparticles $(R=10 \mathrm{~nm})$ and normalized by the absorption of a single particle. From (Raj et al. 1995)

where we have introduced the $N \times N$ block matrix

$$
\boldsymbol{D}_{i j}=\mu_{0} \omega^{2} \mathbb{G}_{0}\left(\mathbf{r}_{i}, \mathbf{r}_{j}\right) .
$$

This relation generalizes expression (29) to arbitrary systems of coupled dipoles. For isotropic and homogeneous particles the generalized vector field of dipolar moments reads

$$
\left(\begin{array}{c}
\mathbf{p}_{1} \\
\vdots \\
\mathbf{p}_{N}
\end{array}\right)=\boldsymbol{A}\left(\begin{array}{c}
\mathbf{E}_{\mathrm{loc}, 1} \\
\vdots \\
\mathbf{E}_{\mathrm{loc}, N}
\end{array}\right)
$$

introducing the block matrix

$$
\boldsymbol{A}_{i j}=\epsilon_{0} \delta_{i j} \underline{\underline{\alpha}}_{i}
$$

where $\underline{\underline{\alpha}}_{i}$ is the electric polarizability tensor associated to the $i^{t h}$ particle. Using Eq. (32), this expression can be reformulated with respect to vectorial incident field as

$$
\left(\begin{array}{c}
\mathbf{p}_{1} \\
\vdots \\
\mathbf{p}_{N}
\end{array}\right)=\boldsymbol{A} \tilde{\boldsymbol{T}}^{-1}\left(\begin{array}{c}
\mathbf{E}_{\mathrm{inc}, 1} \\
\vdots \\
\mathbf{E}_{\mathrm{inc}, N}
\end{array}\right)
$$

with

$$
\tilde{\boldsymbol{T}}_{i j}=\delta_{i j} \mathbb{1}-\left(1-\delta_{i j}\right) k_{0}^{2} \mathbb{G}_{0}\left(\mathbf{r}_{i}, \mathbf{r}_{j}\right) \underline{\underline{\alpha}}_{j} .
$$

This block matrix $\tilde{\boldsymbol{T}}^{-1}$ defines the interplay between all dipoles and the block matrix

$$
\boldsymbol{\alpha}_{\mathrm{dr}}=\frac{1}{\epsilon_{0}} \boldsymbol{A} \tilde{\boldsymbol{T}}^{-1}
$$


also called dressed polarizability (Castanié et al. 2012) results from the multi-scattering process in the set of dipoles. Using the slightly different block matrix

$$
\boldsymbol{T}_{i j}=\delta_{i j} \mathbb{1}-\left(1-\delta_{i j}\right) k_{0}^{2} \underline{\underline{\alpha}}_{i} \mathbb{G}_{0}\left(\mathbf{r}_{i}, \mathbf{r}_{j}\right) .
$$

it can also be expressed as

$$
\boldsymbol{\alpha}_{\mathrm{dr}}=\frac{1}{\epsilon_{0}} \boldsymbol{T}^{-1} \boldsymbol{A},
$$

because $\boldsymbol{T} \boldsymbol{A}=\boldsymbol{A} \tilde{\boldsymbol{T}}$ and $\boldsymbol{T}^{-1} \boldsymbol{A}=\boldsymbol{A} \tilde{\boldsymbol{T}}^{-1}$. This dressed polarizability shows that two types of resonances play a role in the interaction of light with the set of coupled dipoles. The first ones are the resonances of the isolated particles themselves (i.e. the poles of $\underline{\underline{\alpha}}_{i}$ ) while the second (i.e. the poles of the determinant of $\boldsymbol{\alpha}_{\mathrm{dr}}$ or $\boldsymbol{T}^{-1}$ ) are configurational resonances (see Fig. 11) and they depend on the spatial distribution of dipoles. So that the $3 N$ dipolar resonance which are degenerate for spherical nanoparticles, for instance, couple and form a band of $3 N$ resonances in general. Depending on the symmetry in the configuration some of the resonances remain degenerate despite the coupling. A simple example is a chain of nanoparticles. There one finds $N$ two-fold degenerate vertical and $N$ longitudinal resonances (Weber and Ford, 2004) forming bands of coupled modes. A general consequence of this dressing due to the coupling is a broadening of the absorption spectrum in a coupled $N$-dipole system.

\section{Exchanged Power and Poynting vector}

Now we consider the most general situation where the particles are also emitting heat radiation. The fundamental relations to describe heat exchange in a system of $N$ dipoles having temperatures $T_{1}, \ldots, T_{N}$ within the framework of the FE have first been derived in (BenAbdallah et al., 2011). In Ref. (Messina et al., 2013b) the relations for the heat exchange were generalized to treat also the interaction of the $N$ dipolar objects with an environment or background in thermal equilibrium at some temperature $T_{b}$, but only for isotropic dipolar object. Subsequently, these expressions have been extended to anisotropic and non-reciprocal systems taking also the radiation correction into account (Ekeroth et al., 2017, Nikbakht, 2014) and the expression for the mean Poynting vector of such an $N$-dipole system have been determined to quantify its far-field thermal emission (Ekeroth et al., 2017). Finally, in Refs. (Ott and Biehs, 2020 Ott et al. 2019a) the method from Ref. (Messina et al. 2013b) was used to determine the general expressions for the mean Poynting vector and the exchanged heat in a system of $N$ dipoles immersed in an environment at temperature $T_{b}$ which can also be non-reciprocal. A further generalization which takes the possibility of magnetic polarizabilities into account can be found in Ref. (Dong et al. 2017a Manjavacas and de Abajo, 2012). Here we review mainly the derivation of the heat exchange and the mean Poynting vector for $N$ dipolar objects described by an electric polarizability tensor $\underline{\alpha}$ within the framework of (Messina et al. 2013b). This approach is valid for nanoparticles with a size much smaller than the thermal wavelength and for inter-particle distances and distance between the particles and interfaces of the environment larger than twice the diameter (Becerril and Noguez, 2019; Narayanaswamy and Chen, 2008: Otey and Fan, 2011b).

To derive the exchanged power and the mean Poynting vector in an N-dipole system we consider the total electric and magnetic fields

$$
\begin{aligned}
& \mathbf{E}(\mathbf{r}, \omega)=\omega^{2} \mu_{0} \sum_{i=0}^{N} \mathbb{G}^{\mathrm{EE}}\left(\mathbf{r}, \mathbf{r}_{i}\right) \mathbf{p}_{i}+\mathbf{E}^{b}(\mathbf{r}, \omega), \\
& \mathbf{H}(\mathbf{r}, \omega)=\omega^{2} \mu_{0} \sum_{i=0}^{N} \mathbb{G}^{\mathrm{HE}}\left(\mathbf{r}, \mathbf{r}_{i}\right) \mathbf{p}_{i}+\mathbf{H}^{b}(\mathbf{r}, \omega)
\end{aligned}
$$

which are generated by the fluctuational background fields $\mathbf{E}^{b}(\mathbf{r})$ and $\mathbf{H}^{b}(\mathbf{r})$ and the induced and fluctuational dipoles of all particles $(i=1, \ldots, N)$

$$
\mathbf{p}_{i}=\mathbf{p}_{i}^{\text {ind }}+\mathbf{p}_{i}^{\mathrm{H}}
$$

where the induced dipole moments

$$
\mathbf{p}_{i}^{(\text {ind })}=\epsilon_{0} \underline{\underline{\alpha}}_{i} \mathbf{E}\left(\mathbf{r}_{i}\right)
$$

can be expressed in terms of the polarizability tensor $\underline{\underline{\alpha}}_{i}$ of the $\mathrm{i}^{\text {th }}$ dipole. Here we have introduced the electric and magnetic Green functions $\mathbb{G}^{\mathrm{EE}}$ and $\mathbb{G}^{\mathrm{HE}}$ generated by electric dipole moments as defined in Ref. (Eckhardt, 1984) which are now not necessarily the vacuum Green functions, but the general Green functions taking the geometry and material properties of the backround into account. As a consequence the total electric field $\mathbf{E}_{i}=\mathbf{E}\left(\mathbf{r}_{i}\right)$ at the position of the $i$-th dipole is given by the field contributions due to the fluctuating dipole moments $\mathbf{p}_{j}^{\text {fl }}$ of all other dipoles $j \neq i$ and the background field $\mathbf{E}_{i}^{b}=\mathbf{E}^{b}\left(\mathbf{r}_{i}\right)$ including direct thermal emission and multiple scattering. It can be written as (Messina et al. 2013b

$$
\left(\begin{array}{c}
\mathbf{E}_{1} \\
\vdots \\
\mathbf{E}_{N}
\end{array}\right)=\boldsymbol{D} \boldsymbol{T}^{-1}\left(\begin{array}{c}
\mathbf{p}_{1}^{\mathrm{fl}} \\
\vdots \\
\mathbf{p}_{N}^{\mathrm{f}}
\end{array}\right)+\left(\mathbf{1}+\boldsymbol{D} \boldsymbol{T}^{-1} \boldsymbol{A}\right)\left(\begin{array}{c}
\mathbf{E}_{1}^{\mathrm{b}} \\
\vdots \\
\mathbf{E}_{N}^{\mathrm{b}}
\end{array}\right) .
$$

Similarly the induced dipole moments $\mathbf{p}_{i}$ for each particle $i$ can be expressed in terms of the fluctuating dipole moments of all other particles and the background field (Messina et al., 2013b)

$$
\left(\begin{array}{c}
\mathbf{p}_{1} \\
\vdots \\
\mathbf{p}_{N}
\end{array}\right)=\boldsymbol{T}^{-1}\left(\begin{array}{c}
\mathbf{p}_{1}^{\mathrm{f}} \\
\vdots \\
\mathbf{p}_{N}^{\mathrm{f}}
\end{array}\right)+\left(\boldsymbol{T}^{-1} \boldsymbol{A}\right)\left(\begin{array}{c}
\mathbf{E}_{1}^{\mathrm{b}} \\
\vdots \\
\mathbf{E}_{N}^{\mathrm{b}}
\end{array}\right) .
$$


The auxilliary $3 N \times 3 N$-block matrices $\boldsymbol{D}, \boldsymbol{A}$, and $\boldsymbol{T}$ are defined as in Eqs. (34), (36), (40) but with the vacuum Green function $\mathbb{G}_{0}\left(\mathbf{r}_{i}, \mathbf{r}_{j}\right)$ replaced by $\mathbb{G}_{i j}^{\mathrm{EE}}=\mathbb{G}^{\mathrm{EE}}\left(\mathbf{r}_{i}, \mathbf{r}_{j}\right)$ and by $\mathbf{1}_{i j}=\delta_{i j} \mathbb{1}$.

Equipped with this set of expressions it is now possible to derive the dissipated heat in a given dipole $i$ and the mean Poyting vector in a general $N$ dipole system. Analogous to 26 the mean power received by the $\mathrm{i}^{\text {th }}$ dipole is defined as the power dissipated in dipole $i$

$$
\begin{aligned}
\mathcal{P}_{i} & =\left\langle\frac{d \mathbf{p}_{i}(t)}{d t} \cdot \mathbf{E}_{i}(t)\right\rangle \\
& =2 \operatorname{Im} \int_{0}^{\infty} \frac{\mathrm{d} \omega}{2 \pi} \omega\left\langle\mathbf{p}_{i}(\omega) \cdot \mathbf{E}_{i}^{*}(\omega)\right\rangle .
\end{aligned}
$$

Hence by definition the dissipated power inside dipole $i$, i.e. the heat flowing into that dipole, is positive. The mean Poynting vector due to the dipoles and the background fields is given by

$$
\begin{aligned}
\langle\mathbf{S}(\mathbf{r})\rangle & =\langle\mathbf{E}(t) \times \mathbf{H}(t)\rangle \\
& =2 \operatorname{Re} \int_{0}^{\infty} \frac{\mathrm{d} \omega}{2 \pi}\left\langle\mathbf{E}(\mathbf{r}, \omega) \times \mathbf{H}^{*}(\mathbf{r}, \omega)\right\rangle .
\end{aligned}
$$

These expressions already include the fact that the fluctuational fields and dipole moments are stationary so that the mean power and mean Poynting vector do not depend on time. They can be evaluated by assuming that the fluctuational dipole moments and the background fields are in local thermal equilibrium at temperatures $T_{i}(i=1, \ldots, N)$ and $T_{b}$. Then the mean values for the power and Poynting vector which are obviously given by the correlation functions of the fields and the dipole moments can be evaluated by employing the fluctuationdissipation theorem (Kubo, 1966) and assuming that the background fields and the dipole moments are statistically independent, i.e. correlation functions between the background field and the fluctuating dipoles $\left\langle\mathbf{E}^{\mathrm{b}} \otimes \mathbf{p}_{i}\right\rangle$ vanish. For the fields the fluctuation-dissipation theorems are (Agarwal, 1975a)

$$
\begin{aligned}
\left\langle\mathbf{E}_{i}^{\mathrm{b}} \otimes \mathbf{E}_{j}^{\mathrm{b}^{*}}\right\rangle & =2 \omega^{2} \mu_{0} \hbar\left(n_{b}+\frac{1}{2}\right) \frac{\mathbb{G}_{i j}^{\mathrm{EE}}-\mathbb{G}_{j i}^{\mathrm{EE}}}{2 \mathrm{i}}, \\
\left\langle\mathbf{E}_{i}^{\mathrm{b}} \otimes \mathbf{H}_{j}^{\mathrm{b}}\right\rangle & =2 \omega^{2} \mu_{0} \hbar\left(n_{b}+\frac{1}{2}\right) \frac{\mathbb{G}_{i j}^{\mathrm{EH}}-\mathbb{G}_{j i}^{\mathrm{HE}^{\dagger}}}{2 \mathrm{i}}
\end{aligned}
$$

using the notation $\mathbb{G}_{i j}^{\mathrm{EH}}=\mathbb{G}^{\mathrm{EH}}\left(\mathbf{r}_{i}, \mathbf{r}_{j}\right)$ and $\mathbb{G}_{i j}^{\mathrm{HE}}=$ $\mathbb{G}^{\mathrm{HE}}\left(\mathbf{r}_{i}, \mathbf{r}_{j}\right)$. Analoguously, for the dipole moments the fluctuation-dissipation theorem is determined by (Messina et al. 2013b)

$$
\left\langle\mathbf{p}_{i}^{\mathrm{fl}} \otimes \mathbf{p}_{j}^{\mathrm{f} *^{*}}\right\rangle=2 \epsilon_{0} \hbar \delta_{i j}\left(n_{i}+\frac{1}{2}\right) \underline{\underline{\chi}}_{i} .
$$

The generalized susceptibility of the $i^{\text {th }}$ particle is given by (Ekeroth et al., 2017, Herz and Biehs, 2019; Messina et al. $2013 \mathrm{~b}$ )

$$
\underline{\underline{\chi}}_{i}=\frac{\underline{\underline{\alpha}}_{i}-\underline{\underline{\alpha}}_{i}^{\dagger}}{2 \mathrm{i}}-k_{0}^{2} \underline{\underline{\alpha}}_{i} \frac{\mathbb{G}_{i i}^{\mathrm{EE}}-\mathbb{G}_{i i}^{\mathrm{EE}}}{2 \mathrm{i}} \underline{\underline{\alpha}}_{i}^{\dagger} .
$$

The first term of the generalized susceptibility describes simply the intrinsic absorptivity of the dipole, whereas the second term is a radiation correction taking into account that the dipole is coupled to the environment which modifies its absorptivity. In free space this second term simply reads $-k_{0}^{3} /(6 \pi) \underline{\alpha} \underline{\alpha}^{\dagger}$ Ekeroth et al. 2017). Hence,

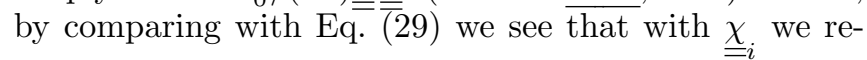
trieve the absorptivity of a dipole $i$ placed in vacuum for the isotropic case $\underline{\underline{\alpha}}_{i}=\alpha_{i} \mathbb{1}$.

Inserting the expressions for the fields and dipole moments into the definitions 48 one obtains for the mean power received by particle $i$ (Ott and Biehs, 2020)

$$
\mathcal{P}_{i}=3 \int_{0}^{\infty} \frac{\mathrm{d} \omega}{2 \pi} \hbar \omega \sum_{j=1}^{N}\left(n_{j}-n_{b}\right) \mathcal{T}_{i j}
$$

where the transmission coefficients are defined as

$$
\mathcal{T}_{i j}=\frac{4}{3} \epsilon_{0} \operatorname{Im} \operatorname{Tr}\left[\boldsymbol{T}_{i j}^{-1} \underline{\underline{\chi}}_{j}\left(\boldsymbol{D} \boldsymbol{T}^{-1}\right)_{i j}^{\dagger}\right]
$$

Equation (54) is the general expression for the dissipated power or heat flowing into a dipole at temperature $T_{i}$ surrounded by $N-1$ dipoles at temperatures $T_{j}(j \neq i)$ described by an anisotropic or even non-reciprocal polarizability immersed in a general environment or background at temperature $T_{b}$ which can itself be anisotropic or non-reciprocal, properties which are taken into account via the polarizability and the Green function. In general, if either the dipole or the background or both are non-reciprocal one has $\mathcal{T}_{i j} \neq \mathcal{T}_{j i}$ (Herz and Biehs, 2019, Zhu et al. 2018). It should be noted that in the literature a variety of different equivalent expressions for the transmission coefficients $\mathcal{T}_{i j}$ can be found as for instance in (Ben-Abdallah et al., 2011, Ekeroth et al. 2017, Messina et al. 2013b Nikbakht, 2014, Ott and Biehs, 2020 Ott et al. 2019a). Finally, when replacing $n_{j}-n_{b}$ by $n_{j}-n_{i}+n_{i}-n_{b}$ Eq. (54) can be recast into the more intuitive form (Messina et al. 2013b)

$$
\mathcal{P}_{i}=3 \int_{0}^{\infty} \frac{\mathrm{d} \omega}{2 \pi} \hbar \omega\left(\sum_{j \neq i}\left(n_{j}-n_{i}\right) \mathcal{T}_{i j}+\left(n_{i}-n_{b}\right) \mathcal{T}_{i b}\right)
$$

with $\mathcal{T}_{i b}=\sum_{j} \mathcal{T}_{i j}$. This formula has the advantage that it clearly expresses the power dissipated into dipole $i$ by the power exchanged between dipole $i$ and all the other dipoles and the power of dipole $i$ exchanged with the environment.

Similarly, by starting with the definition of the mean Poynting vector in 49 one obtains for the spectral heat flux for the $N$ fluctuating dipoles immersed in a back- 
ground Ott and Biehs, 2020

$$
\begin{aligned}
\left\langle S_{\omega, \alpha}\right\rangle= & 4 \hbar \omega^{2} \mu_{0} k_{0}^{2} \sum_{\beta, \gamma=x, y, z} \epsilon_{\alpha \beta \gamma} \operatorname{Re}[ \\
& \sum_{j=1}^{N}\left(n_{j}-n_{b}\right) \sum_{i=1}^{N}\left(\mathbb{G}_{0 i}^{\mathrm{EE}} \boldsymbol{T}_{i j}^{-1}\right) \underline{\underline{\chi}}_{j} \sum_{k=1}^{N}\left(\mathbb{G}_{0 k}^{\mathrm{HE}} \boldsymbol{T}_{k j}^{-1}\right)^{\dagger} \\
& +\frac{n_{b}}{2 \mathrm{i}} \sum_{i, j=1}^{N}\left(\mathbb{G}_{0 i}^{\mathrm{EE}} \boldsymbol{T}_{i j}^{-1} \underline{\underline{\alpha}}_{j} \mathbb{G}_{j 0}^{\mathrm{EH}}-\left(\mathbb{G}_{0 i}^{\mathrm{HE}} \boldsymbol{T}_{i j}^{-1} \underline{\underline{\alpha}}_{j} \mathbb{G}_{j 0}^{\mathrm{EE}}\right)^{\dagger}\right) \\
& \left.+\frac{n_{b}}{k_{0}^{2}}\left(\frac{\mathbb{G}_{00}^{\mathrm{EH}}-\mathbb{G}_{00}^{\mathrm{HE}}{ }^{\dagger}}{2 \mathrm{i}}\right)\right]_{\beta \gamma}
\end{aligned}
$$

where $\epsilon_{\alpha \beta \gamma}$ is the Levi-Civita tensor and $\mathbb{G}_{0 i}^{\mathrm{EE}}=$ $\mathbb{G}^{\mathrm{EE}}\left(\mathbf{r}, \mathbf{r}_{i}\right), \mathbb{G}_{00}^{\mathrm{EE}}=\mathbb{G}^{\mathrm{EE}}(\mathbf{r}, \mathbf{r})$, etc. The first term describes the heat flux emitted by the particles into the background, the last term describes the heat flux of the background fields without the dipoles, and the second term describes the interference of the background fields due to the presence of the dipoles. In the case that the background geometry fulfills Lorentz reciprocity (Caloz et al. 2018 the last terms vanishes since then $\mathbb{G}_{i j}^{\mathrm{EH}}{ }^{\dagger}=$ $-\mathbb{G}_{j i}^{\mathrm{HE}}$. This simply means that if we have no dipoles the mean heat flux in the background $\left\langle\mathbf{S}^{b}\right\rangle=\left\langle\mathbf{E}^{b}(t) \times \mathbf{H}^{b}(t)\right\rangle$ which is at local thermal equilibrium vanishes. On the other hand, as shown by Silvereinha (Silveirinha, 2017) for a non-reciprocal background there can be a nonvanishing mean Poynting vector even in thermal equilibrium.

In certain cases the heat flux between the dipolar objects is dominant so that the emission into the background is negligibly small. If for example the dipoles are placed into a vacuum at temperature $T_{b}$ then the power exchanged between the dipoles is for distances much smaller than the thermal wavelength, i.e. in the near-field regime, much larger then the power exchange with the background (Messina et al. 2013b). When placing the dipolar objects, for instance, close to a substrate then the inter-dipole heat exchange is still dominating if the distance between the dipoles is much smaller than the distance to the substrate (Ott and Biehs, 2020). In such situations, the $N$-dipole system can also be treated as a closed system. This can be done by neclegting in the above expressions the heat exchange between the dipoles and the background and the heat flux due to the background fields so that

$$
\mathcal{P}_{i}=3 \int_{0}^{\infty} \frac{\mathrm{d} \omega}{2 \pi} \hbar \omega \sum_{j \neq i}\left(n_{j}-n_{i}\right) \mathcal{T}_{i j}
$$

and

$$
\begin{aligned}
\left\langle S_{\omega, \alpha}\right\rangle= & 4 \hbar \omega^{2} \mu_{0} k_{0}^{2} \sum_{\beta, \gamma=x, y, z} \epsilon_{\alpha \beta \gamma} \sum_{j=1}^{N} n_{j} \\
& \times \operatorname{Re}\left[\sum_{i=1}^{N}\left(\mathbb{G}_{0 i}^{\mathrm{EE}} \boldsymbol{T}_{i j}^{-1}\right) \underline{\underline{\chi}}_{j} \sum_{k=1}^{N}\left(\mathbb{G}_{0 k}^{\mathrm{HE}} \boldsymbol{T}_{k j}^{-1}\right)^{\dagger}\right]_{\beta \gamma} .
\end{aligned}
$$

Note that, even though $\mathcal{P}_{i}$ contains only the power dissipated in dipole $i$ due to the heat exchange with all other dipoles, the mean Poynting vector includes also the thermal radiation of all dipoles into their background which is assumed to have zero temperature. To be fully consistent with the assumption that the background is simply removed from the description the second term in the generalized susceptibility $\underline{\underline{\chi}}_{j}$ in Eq. 53 might be neglected. For systems where the dipole approximation is valid this term is typically very small and can therefore often be neglected anyway.

The same equations can be obtained by neglecting in the derivation right from the start any contribution from the background fields. In this case $\mathcal{P}_{i}$ can also be obtained by considering the power exchanged between all pairs of dipoles, only, as originally done in many works as for instance in Ref. (Ben-Abdallah et al., 2011). To this end, the heat dissipated in dipole $i$ due to a fluctuational field $\mathbf{E}_{i j}=\left(\boldsymbol{D} \boldsymbol{T}^{-1}\right)_{i j} \mathbf{p}_{j}^{\mathrm{fl}}$ generated by a fluctuational dipole $\mathbf{p}_{j}^{\mathrm{ff}}$ is considered as the power flow from dipole $j$ to $i$ yielding

$$
\begin{aligned}
\mathcal{P}_{j \rightarrow i} & =\left\langle\frac{d \mathbf{p}_{i}(t)}{d t} \cdot \mathbf{E}_{i j}(t)\right\rangle \\
& =3 \int_{0}^{\infty} \frac{\mathrm{d} \omega}{2 \pi} \hbar \omega n_{j} \mathcal{T}_{i j}(\omega) .
\end{aligned}
$$

Then the power dissipated by the $i^{\text {th }}$ dipole is just the sum of the power flowing between dipole $i$ and the other objects

$$
\begin{aligned}
\mathcal{P}_{i} & =\sum_{j \neq i}\left(\mathcal{P}_{j \rightarrow i}-\mathcal{P}_{i \rightarrow j}\right) \\
& =\sum_{j \neq i} 3 \int_{0}^{\infty} \frac{\mathrm{d} \omega}{2 \pi} \hbar \omega\left(n_{j} \mathcal{T}_{i j}(\omega)-n_{i} \mathcal{T}_{j i}(\omega)\right) .
\end{aligned}
$$

Since in thermal equilibrium $\mathcal{P}_{i}=0$ we can derive the condition (Latella and Ben-Abdallah, 2017, Ott et al. 2019a)

$$
\sum_{j \neq i} \mathcal{T}_{i j}(\omega)=\sum_{j \neq i} \mathcal{T}_{j i}(\omega)
$$

This condition simply expresses the fact that even though $T_{i j} \neq T_{j i}$ in general, the heat flux from $i$ to all other dipoles [rhs of [62] ] must be the same as the heat flow from all other dipoles to $i$ [lhs of [62] ] in equilibrium. By inserting this equilibrium condition into the second term of (61) we retrieve (58). 


\section{Non-additivity in many-dipole systems}

Before we discuss the non-additivity of the power exchange in a $N$-dipole system based on Eq. (60), let us focus on the power exchange between two dipoles $(N=2)$. The first derivation of the heat exchange between two dipolar objects within the framework of FE was given in (Volokitin and Persson, 2001) and extended to take magnetic dipole moments into account (Chapuis et al., 2008a. Manjavacas and de Abajo, 2012) as well as multipolar contributions (Becerril and Noguez, 2019, Pérez-Madrid et al., 2008). A quantum dynamical description can be

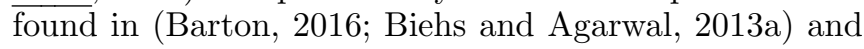
a discussion of different prefactors found in the literature in (Dedkov and Kyasov, 2011; Sasihithlu, 2019). Using our expression in Eq. (60) for $N=2$ and temperatures $T_{1} \neq 0 \mathrm{~K}$ and $T_{2}=0 \mathrm{~K}$ we obtain for the power received by dipole 2

$$
\mathcal{P}_{1 \rightarrow 2}=3 \int_{0}^{\infty} \frac{\mathrm{d} \omega}{2 \pi} \hbar \omega n_{1} \mathcal{T}_{21}
$$

The transmission coefficient $\mathcal{T}_{12}$ can be expressed as

$$
\mathcal{T}_{21}=\frac{4}{3} k_{0}^{4} \operatorname{Im} \operatorname{Tr}\left[\mathbb{D}^{-1} \mathbb{G}_{21} \underline{\underline{\chi}}_{1}\left(\mathbb{D}^{-1} \mathbb{G}_{21}\right)^{\dagger} \underline{\underline{\chi}}_{2}\right] \text {. }
$$

with $\mathbb{D}=\left(\mathbb{1}+k_{0}^{4} \mathbb{G}_{21} \underline{\underline{\alpha}}_{1} \mathbb{G}_{12} \underline{\underline{\alpha}}_{2}\right)$ introducing the generalized susceptibility

$$
\underline{\underline{\tilde{\chi}}}_{2}=\frac{\underline{\underline{\alpha}}_{2}-\underline{\underline{\alpha}}_{2}^{\dagger}}{2 \mathrm{i}}-k_{0}^{2} \underline{\underline{\alpha}}_{2}^{\dagger} \frac{\mathrm{G}_{22}-\mathbb{G}_{22}^{\dagger}}{2 \mathrm{i}} \underline{\underline{\alpha}}_{2} .
$$

Note that this general susceptibility only differs slightly from the definition (53), whereas for isotropic dipoles both definitions coincide. This is the most general expression of the transmission coefficient for two dipolar objects in a given environment of any shape. The appearance of the terms $\mathbb{D}^{-1}$ in the transmission coefficient are due to multiple interactions between the dipoles. Therefore the hybridization of any localized dipole resonance due to the strong coupling for small distances is accounted for in this expression. Note that Eq. (64) resembles Eq. (36) of (Ekeroth et al. 2017) but with the slight difference that in that work $\underline{\underline{\chi}}_{2}$ is used instead of $\underline{\underline{\chi}}_{2}$. On the other hand, the form of the transmission coefficient (64) has also been found in (Herz and Biehs, 2019, Krüger et al. 2012 ) within the scattering approach of (Krüger et al. 2012). However, within the range of validity of the dipole approximation the second term in $\underline{\chi}$ or $\underline{\tilde{\chi}}$ typically can be neglected and many works simply use

$$
\underline{\underline{\chi}}_{i} \approx \underline{\underline{\underline{\chi}}}_{i}=\frac{\underline{\underline{\alpha}}_{i}-\underline{\underline{\alpha}}_{i}^{\dagger}}{2 \mathrm{i}}
$$

Now, when adding a third dipole at $T_{3}=0 \mathrm{~K}$ then we still can use (63) and 64 to quantify the power exchanged between dipole 1 and 2 . The main difference is that $\boldsymbol{T}_{12}^{-1}$ now also contains the coupling with the third dipole. Hence the sheer presence of the third particle

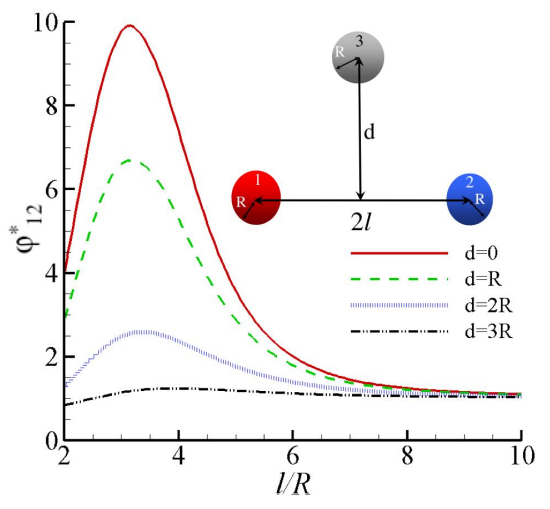

FIG. 12 Power flow exchanged between two SiC nanoparticles at $T_{1}=300 \mathrm{~K}$ (red) and at $T_{2}=0 \mathrm{~K}$ (blue) in presence of a third SiC nanoparticle at temperature $T_{3}=0 \mathrm{~K}$ (grey) and normalized by the power exchanged between two isolated particles, i.e. $\varphi_{12}^{*}=\mathcal{P}_{1 \rightarrow 2}\left(T_{1}, T_{2}, T_{3}\right) / \mathcal{P}_{1 \rightarrow 2}\left(T_{1}, T_{2}\right)$. From $($ BenAbdallah et al. 2011).
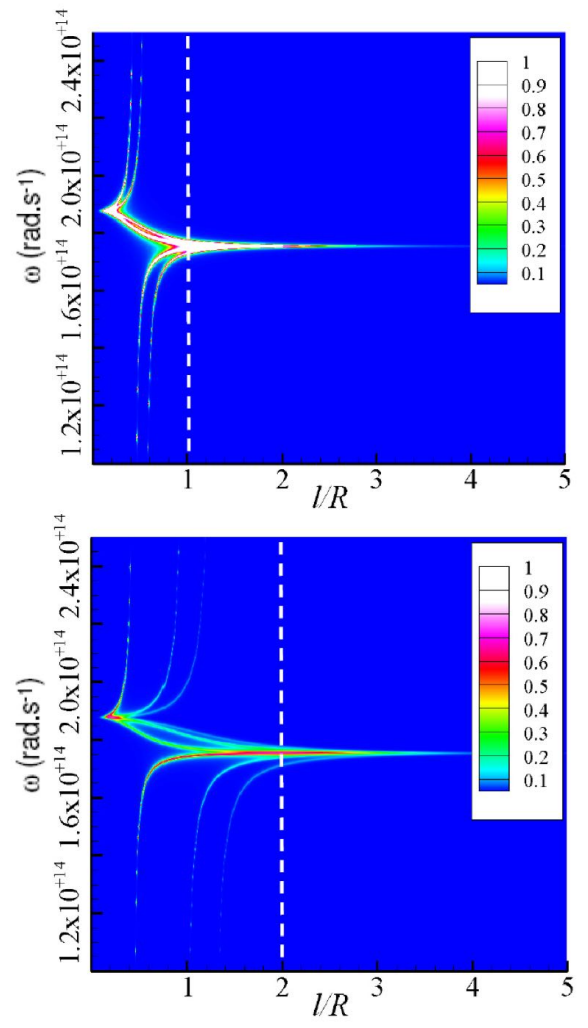

FIG. 13 Transmission coefficient $\mathcal{T}_{21}$ between (a) two $\mathrm{SiC}$ nanoparticles and (b) between two $\mathrm{SiC}$ nanoparticles in presence of a third $\mathrm{SiC}$ nanoparticles as in Fig. 12 for $d=0$. The dashed line marks the region where the particles would touch. The unphysical region beyond this line is shown to illustrate the hybridization mechanism of dipolar resonances, which can be nicely seen in that region. From (Ben-Abdallah et al. 2011). 
changes the transmission coefficients due to the fact that it changes the mode structure which is for dipoles with a localized resonance again due to the hybridization for three dipoles this time (see Fig. 13 responsible for the broadening of the absorption spectrum as discussed in Sec. III.A.1. As a consequence, the presence of a third dipole changes the power exchange $\mathcal{P}_{1 \rightarrow 2}$ proving that the heat exchange in an $N$-dipole system is non-additive. This formalism is only valid for interparticles distances larger than $4 R, R$ being the radius of the particles. It can be extended to smaller distances by including multipolar contributions (Czapla and Narayanaswamy, 2019).

This many-body effect can be exploited to enhance for example the exchanged power between two dipolar objects 1 and 2 by bridging the distance via a third dipole which is placed between 1 and 2 as shown in (BenAbdallah et al. 2011) (see Fig. 12). However, it should be kept in mind that the heat flux between two dipoles in a $N$-dipole system cannot be arbitrarily enhanced. As discussed in (Ben-Abdallah et al., 2011) it can be easily shown that each of the conductance between two dipoles can be at most 3 times the quantum of thermal conductance. Nonetheless, this upper limit is difficult to achieve leaving much space for optimmizations. Several works have shown that it is possible to tailor the inter-dipole heat flux via a third dipole or third object. For example, (Messina et al. 2013b has studied the relaxation dynamics for the three-body configuration and (Dong et al. 2017a) have also included the possibility to have a magnetic polarizability as needed to describe metallic nanoparticles in the infrared. Furthermore, using prolate (Incardone et al., 2014; Nikbakht, 2014, 2015) or oblate (Choubdar and Nikbakht, 2016) spheroidal nanoparticles it has been demonstrated that by changing the relative orientation of the nano-particles and in particular an intermediate nanoparticle the heat flux can be switched and enhanced efficiently (see also Fig. 31. Furthermore, the coupling of two nanoparticles via the surface modes of an interface or intermediate medium has been studied as discussed in detail in Sec. III.C.3. Finally, the non-additivity of the heat exchange has consequences for the transport properties in nano-particle chains and complex nanoparticle networks as discussed in detail in Sec. III.C.2.

\section{T-DDA (as example of application)}

The expressions for the heat exchange in systems with $N$ dipolar objects in (58) without the contribution of the background as derived by (Ben-Abdallah et al. 2011) have been employed first by (Edalatpour and Francoeur, 2014) to determine the heat exchange between macroscopic objects with isotropic and later by (Ekeroth et al. 2017) for macroscopic objects with anisotropic and magneto-optical material properties. The idea is to replace the macroscopic objects by a great number $N$ of small cubes of volume $V_{i}(i=1, \ldots, N)$ which can be approximated as dipoles with the corresponding polarizabilities. In Ref. (Ekeroth et al., 2017) the polarizability including the radiative corrections, as rederived by (Albaladejo et al. 2010) and originally also used by (Draine, 1988), writes

$$
\underline{\underline{\alpha}}_{i}=\left(\mathbb{1}-i \frac{k_{0}^{3}}{6 \pi} \underline{\underline{\alpha}}_{0 i}\right)^{-1} \underline{\underline{\alpha}}_{0 i}
$$

in terms of the quasistatic polarizability

$$
\underline{\underline{\alpha}}_{0 i}=3 V_{i}(\varepsilon-\mathbb{1})(\varepsilon+2 \mathbb{1})^{-1} .
$$

Note that in Ref. (Edalatpour and Francoeur, 2014) another expression for the dressed polarizability has been used known as the strong form of the coupled dipole method. A detailed discussion on the different expressions of the dressed polarizabilities in the context of classical coupled dipole method (Purcell and Pennypacker. 1973) has been given by (Lakhtakia, 1992).

This method known as discrete dipole approximation (DDA) for describing thermal radiation phenomena between macroscopic objects has been coined (Edalatpour and Francoeur, 2014) thermal discrete dipole approximation (T-DDA). It has been succesfully employed to determine the heat flux between macroscopic reciprocal and non-reciprocal cubes and spheres (Edalatpour and Francoeur, 2014, Edalatpour et al. 2015: Ekeroth et al., 2018, 2017), and also for the heat flux between a sharp conical tip and a planar substrate (Edalatpour and Francoeur, 2016). In principle this method can also be used to determine the heat flux between two macroscopic objects in arbitrary many-body systems. As discussed in (Edalatpour et al. 2015) in detail, the large number of dipolar subvolumes needed to describe macroscopic objects or have a convergent numerical result sets a certain limit to this numerical method. See also the discussion in Sec. II.F.

Finally, the TDDA method also allows for determining the thermal emission of macroscopic objects by calculation of the mean Poynting vector from Eq. (59) in the far-field regime (Ekeroth et al. 2017). This can also be done with a standard DDA by determing the absorptivity as discussed in Sec. III.A.1 of the macroscopic object modelled by an assembly of dipoles and then using the Kirchhoff law to determine the emissivity. Now, the main advantage of the TDDA is that it allows to attribute to each volume element a given temperature. Hence, TDDA opens up the possibility to calculate thermal emission of macroscopic objects with a given temperature distribution, whereas the standard DDA can only handle emission of isothermal objects or dipolar assemblies. Note, that the assumption of local thermal equilibrium sets strict bounds to the spatial variation of temperature distributions (Eckhardt, 1984). 

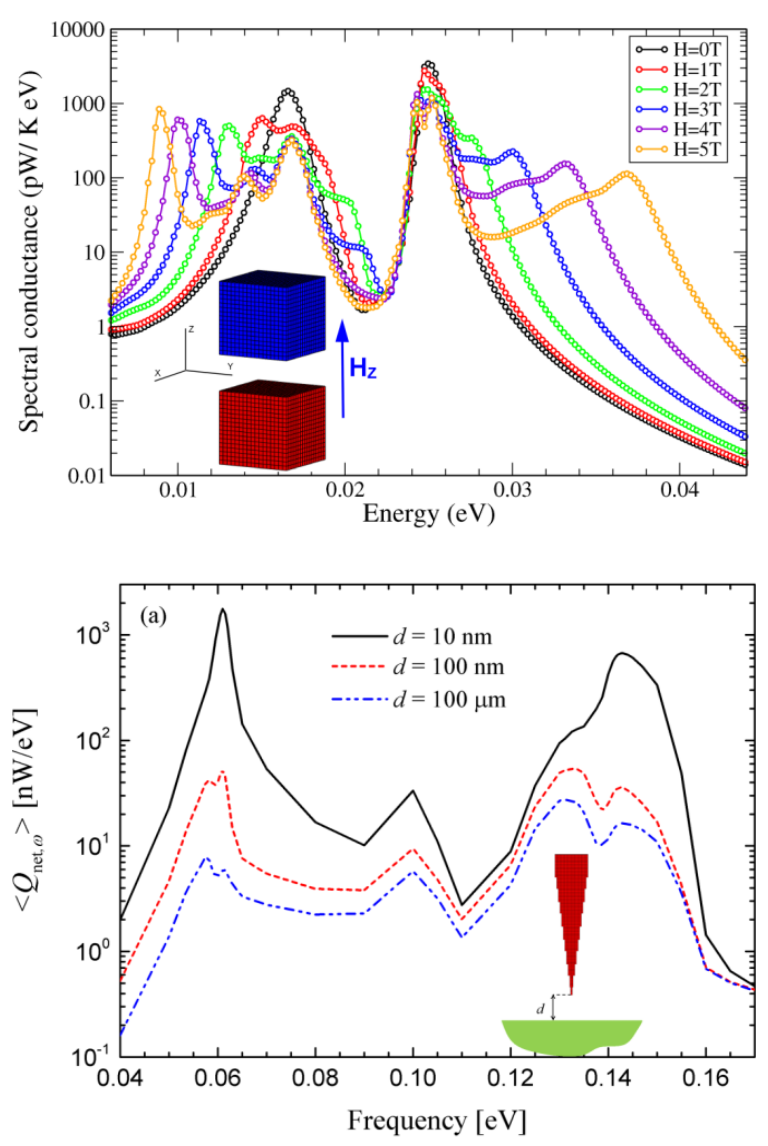

FIG. 14 (a) Spectral conductance as a function of the energy for InSb cubes with a cube side of 1 micron separated by a 500 $\mathrm{nm}$ gap, at $\mathrm{T}=300 \mathrm{~K}$, and for various values of the magnetic field $\mathrm{H}$ applied along the $\mathrm{z}$ direction. The inset shows the discretization geometry: the number of dipoles per cube is 4913 (each one has an edge length of $59 \mathrm{~nm}$ ). From (Ekeroth et al. 2017). (b) Spectral heat flux between a silica probe and silica surface. From (Edalatpour and Francoeur. 2016).

\section{B. Heat flux in macroscopic many-body systems}

In the last section we have described a formalism allowing to account for the heat exchange in an arbitrary set of dipolar particles. As clarified above, although formally and computationally simpler, this framework is limited in terms of distance between the particles. For this reason, in the last decade several theoretical schemes have been developed to account for the heat transfer in configurations of two or more macroscopic bodies. The purpose of these techniques is to address bodies with in principle arbitrary geometry and optical properties. As we have seen in Secs. II.E and II.F, several techniques have been introduced to successfully treat this problem. We are going to focus here on scattering-matrix techniques, where each macroscopic body is described in terms of its scattering operators, accounting for its response to an incoming electromagnetic field.

\section{Scattering-matrix formalism}

Two closely-related formalisms based on this approach have been introduced between 2009 and 2011 by Bimonte (Bimonte, 2009), Krüger and collaborators (Krüger et al., 2012, 2011) and Messina and Antezza (Messina and Antezza, 2011a b). The main difference between the these works is that Krüger et al. derive expressions which are suitable to any choice of basis for the electromagnetic field, while Messina and Antezza explicitly use a plane-wave basis, thus providing more explicit (albeit less general) expressions in terms of the individual scattering operators. In order to define these operators, the electric field in any region of the system is decomposed in plane waves as

$$
\begin{aligned}
\mathbf{E}^{\phi}(\mathbf{r}, t)=2 \operatorname{Re} & {\left[\sum_{p} \int_{0}^{+\infty} \frac{\mathrm{d} \omega}{2 \pi} \int \frac{\mathrm{d}^{2} \boldsymbol{\kappa}}{(2 \pi)^{2}} \exp \left[\mathrm{ik}^{\phi} \cdot \mathbf{r}\right]\right.} \\
\times & \left.\exp [-\mathrm{i} \omega t] \hat{\boldsymbol{\epsilon}}_{p}^{\phi}(\boldsymbol{\kappa}, \omega) E_{p}^{\phi}(\boldsymbol{\kappa}, \omega)\right],
\end{aligned}
$$

where $\omega$ is the frequency, $\boldsymbol{\kappa}=\left(k_{x}, k_{y}\right)$ the projection of the wavevector on the $x-y$ plane, $p$ the polarization index, taking values 1 (transverse electric) and 2 (transverse magnetic), $\phi$ the propagation direction along the $z$ axis. Moreover, $\mathbf{k}^{\phi}=\left(\boldsymbol{\kappa}, \phi k_{z}\right)$ is the full wavevector, while the unit polarization vectors are defined as follows:

$$
\begin{aligned}
\hat{\boldsymbol{\epsilon}}_{\mathrm{TE}}^{\phi}(\boldsymbol{\kappa}, \omega) & =\hat{\mathbf{z}} \times \hat{\boldsymbol{\kappa}}=\frac{1}{\kappa}\left(-k_{y} \hat{\mathbf{x}}+k_{x} \hat{\mathbf{y}}\right) \\
\hat{\boldsymbol{\epsilon}}_{\mathrm{TM}}^{\phi}(\boldsymbol{\kappa}, \omega) & =\frac{c}{\omega}\left(-\kappa \hat{\mathbf{z}}+\phi k_{z} \hat{\boldsymbol{\kappa}}\right) .
\end{aligned}
$$

Each body is described in terms of four scattering operators $\mathcal{R}^{\phi}(\omega)$ and $\mathcal{T}^{\phi}(\omega)(\phi=+,-)$, connecting the amplitudes $E_{p}^{\phi}(\boldsymbol{\kappa}, \omega)$ of the incoming and scattered fields, as (suppressing the frequency arguments)

$$
\begin{aligned}
& E_{p}^{(\mathrm{re}) \phi}(\boldsymbol{\kappa})=\sum_{p^{\prime}} \int \frac{\mathrm{d}^{2} \boldsymbol{\kappa}^{\prime}}{(2 \pi)^{2}}\left\langle p, \boldsymbol{\kappa}\left|\mathcal{R}^{\phi}\right| p^{\prime}, \boldsymbol{\kappa}^{\prime}\right\rangle E_{p^{\prime}}^{(\mathrm{in})-\phi}\left(\boldsymbol{\kappa}^{\prime}\right), \\
& E_{p}^{(\mathrm{tr}) \phi}(\boldsymbol{\kappa})=\sum_{p^{\prime}} \int \frac{d^{2} \boldsymbol{\kappa}^{\prime}}{(2 \pi)^{2}}\left\langle p, \boldsymbol{\kappa}\left|\mathcal{T}^{\phi}\right| p^{\prime}, \boldsymbol{\kappa}^{\prime}\right\rangle E_{p^{\prime}}^{(\mathrm{in}) \phi}\left(\boldsymbol{\kappa}^{\prime}\right),
\end{aligned}
$$

where each mode $(\omega, \boldsymbol{\kappa}, p)$ of the scattered field has in general components from each mode $\left(\omega, \boldsymbol{\kappa}^{\prime}, p^{\prime}\right)$ of the incoming field, the frequency $\omega$ being conserved since we are addressing only stationary processes. The action of these operators is schematically represented in Fig. 15.

At this stage, it is interesting to sketch the main steps and assumptions leading to the expression of the radiative heat flux on each body, which can be summarized as follows:

1. The fields generated by the fluctuating charges inside each body are identified as the source fields, along with the environmental field in which the system is embedded. 


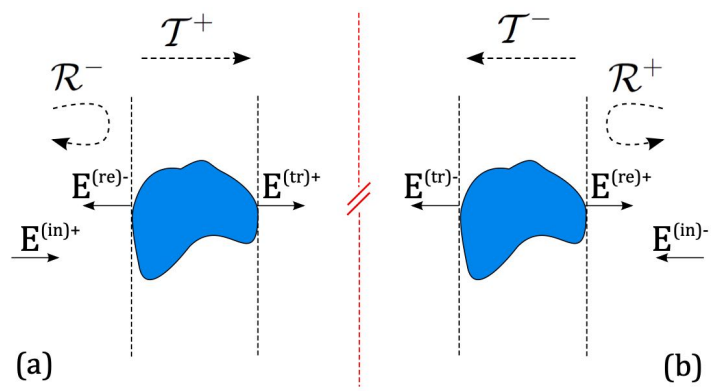

FIG. 15 Definition of reflection and transmission operators associated with an individual body. From (Messina and Antezza, 2011b).

2. The correlation functions of the individual source fields are deduced from the assumption of local thermal equilibrium.

3. The total field in each region is explicitly written, in terms of the source fields, as a result of the scattering (reflection and transmission) processes occurring due to the presence of the bodies.

4. The correlation functions of the total field in each region can be deduced.

5. These are used for the calculation of the average value of the Poynting vector.

We stress that in point 2 the assumption of local thermal equilibrium is equivalent to stating that the statistical properties of the field emitted by each body are the same we would have if the body was at thermal equilibrium at its own temperature. The details about the derivation of such correlation functions can be found in Ref. (Messina and Antezza, 2011b). This step leads to a source correlation function equivalent to Eq. 52 already seen in the case of dipoles, with the difference that in this case the scattering operator, accounting for the geometric and optical properties of the body, will explicitly appear.

The steps described above allow to explicitly write the power absorbed by each body $i$ under the form

$$
\mathcal{P}_{i}=\operatorname{Tr}\left[\hbar \omega\left(\sum_{j \neq i}\left(n_{j}-n_{i}\right) \mathcal{T}_{i j}+\left(n_{i}-n_{b}\right) \mathcal{T}_{i b}\right)\right],
$$

analogous to Eq. (56) already encountered in the dipolar case, where the trace operator is defined as

$$
\operatorname{Tr} \mathcal{A}=\sum_{p} \int \frac{\mathrm{d}^{2} \boldsymbol{\kappa}}{(2 \pi)^{2}} \int_{0}^{+\infty} \frac{\mathrm{d} \omega}{2 \pi}\langle p, \boldsymbol{\kappa}|\mathcal{A}| p, \boldsymbol{\kappa}\rangle .
$$

We focus here on the contribution to the heat flux on body 1 associated with the presence of body 2 . The corresponding transmission coefficient $\mathcal{T}_{12}$ reads

$$
\mathcal{T}_{12}=U^{(2,1)} \chi_{2} U^{(2,1) \dagger} \tilde{\chi}_{1}
$$

where $U^{(2,1)}=\left(1-\mathcal{R}^{(2)-} \mathcal{R}^{(1)+}\right)^{-1}$ is the operator decribing the infinite series of reflections inside the cavity formed by bodies 1 ans 2 and the generalized susceptibilities are defined as

$$
\begin{aligned}
& \chi_{2}=f_{-1}\left(\mathcal{R}^{(2)-}\right)-\mathcal{T}^{(2)-} P_{-1}^{(\mathrm{pw})} \mathcal{T}^{(2)-\dagger} \\
& \tilde{\chi}_{1}=f_{1}\left(\mathcal{R}^{(1)+}\right)-\mathcal{T}^{(1)-\dagger} \mathcal{P}_{1}^{(\mathrm{pw})} \mathcal{T}^{(1)-}
\end{aligned}
$$

by means of the auxiliary functions

$f_{\alpha}(\mathcal{R})=\left\{\begin{array}{c}\mathcal{P}_{-1}^{(\mathrm{pw})}-\mathcal{R} \mathcal{P}_{-1}^{(\mathrm{pw})} \mathcal{R}^{\dagger}+\mathcal{R} \mathcal{P}_{-1}^{(\mathrm{ew})}-\mathcal{P}_{-1}^{(\mathrm{ew})} \mathcal{R}^{\dagger} \\ \alpha=-1 \\ \mathcal{P}_{1}^{(\mathrm{pw})}-\mathcal{R}^{\dagger} \mathcal{P}_{1}^{(\mathrm{pw})} \mathcal{R}+\mathcal{R}^{\dagger} \mathcal{P}_{1}^{(\mathrm{ew})} s-\mathcal{P}_{1}^{(\mathrm{ew})} \mathcal{R} \\ \alpha=1\end{array}\right.$.

The operators $\mathcal{P}_{n}^{(\mathrm{pw})}$ and $\mathcal{P}_{n}^{(\mathrm{ew})}$ defined (for any integer $n)$ as

$$
\left\langle p, \boldsymbol{\kappa}\left|\mathcal{P}_{n}^{(\mathrm{pw} / \mathrm{ew})}\right| p^{\prime}, \boldsymbol{\kappa}^{\prime}\right\rangle=k_{z}^{n}\left\langle p, \boldsymbol{\kappa}\left|\Pi^{(\mathrm{pw} / \mathrm{ew})}\right| p^{\prime}, \boldsymbol{\kappa}^{\prime}\right\rangle,
$$

where $\Pi^{(\mathrm{pw})}=\Theta(\omega-c k)$ and $\Pi^{(\mathrm{ew})}=\Theta(c k-\omega)$ are the projectors on the propagative and evanescent sector, respectively. The transmission coefficient $\mathcal{T}_{12}$ has the same form as in Eq. 64 for two dipolar objects. By choosing the T-operator for dipolar objects or using the plane wave expansion of the T-operators both forms of transmission coefficients can be obtained from the general T-operator expression in (Herz and Biehs, 2019, Krüger et al., 2012).

This approach was later generalized to the case of three arbitrary bodies (Messina and Antezza, 2014). The Landauer-like expression (72) of the power absorbed by each body remains valid, meaning that e.g. the flux on body 1 has contributions coming from bodies 2 and 3, as well as from the environment. It is interesting to investigate here the expression of the transmission coefficient $\mathcal{T}_{12}$ between bodies 1 and 2 in this three-body configuration. It reads

$$
\begin{aligned}
\mathcal{T}_{12} & =U^{(23,1)}\left(f_{-1}\left(\mathcal{R}^{(23)-}\right)-\mathcal{T}^{(2)-} U^{(3,2)} f_{-1}\left(\mathcal{R}^{(3)-}\right)\right. \\
& \left.\times U^{(3,2) \dagger} \mathcal{T}^{(2)-\dagger}\right) U^{(23,1) \dagger} \tilde{\chi}_{1},
\end{aligned}
$$

in which a two-body reflection operator (and the associated multi-reflection operator $U^{(23,1)}$ ) appears, defined as

$$
\mathcal{R}^{(23)-}=\mathcal{R}^{(2)-}+\mathcal{T}^{(2)-} U^{(3,2)} \mathcal{R}^{(3)-} \mathcal{T}^{(2)+}
$$

We immediately see that Eqs. (74) and $(79)$ are different. The important message behind this comparison is that as for the dipolar case discussed in Sec. III.A.3 not only does the presence of body 3 introduce an additional source for the energy transfer on body 1 , but it modifies the transmission coefficient $\mathcal{T}_{12}$, and consequently the way bodies 1 and 2 exchange heat. In other words, the third body in the system acts both as a source/sink of radiation and 
as a scatterer (independently of its temperature), modifying the transmission amplitudes of other channels. We conclude that Eq. $\sqrt{79}$ is by itself a proof and a quantitative evaluation of the non-additive nature of RHT, in the simplest possible many-body system made of three bodies.

The same approach described in Sec. III.B and applied both to two- and three-body systems has been generalized to some years later to the case of $N$ bodies (Latella et al. 2017). In this case, for the sake of simplicity, only planar bodies, i.e. parallel slabs of finite thickness separated by vacuum gaps, have been considered. This assumptions has two main advantages: first, the plane-wave development is particularly convenient for this configuration, since it fully suits its symmetry; moreover the translational invariance along the transverse coordinates makes all the scattering operators diagonal with respect to both $p$ and $\boldsymbol{\kappa}$, significantly simplifying all the expressions. We stress that, since we are dealing here with infinite systems, the power on each body has to be replaced with the heat flux $\Phi$ it receives (power per unit surface).

\section{Non-additivity in many-body systems}

In the last Section, we have analytically shown the nonadditivity of RHT. In the simplest case of three bodies, the appearance of the third one modifies the transmission amplitude $\mathcal{T}_{12}$, namely the way in which bodies 1 and 2 exchange energy. This is shown by the comparison of Eqs. (74) and (79). Apart from this formal comparison, it is interesting to address quantitatively the modification to the energy flux between bodies 1 and 2 due to the introduction of a third body in the system. This analysis has been performed analogous to the configuration discussed in III.A.3 by (Müller et al. 2017), where the authors generalize the formalism developed in (Krüger et al. 2012), already valid in the scenario of $N$ bodies, to the case of the presence of a nonabsorbing background medium. In this work, the authors apply their formalism to the calculation of RHT between two SiC planar slabs (bodies 1 and 2) separated by a vacuum gap of thickness $d$, when a particle of polarizability $\alpha$ (assumed to be nondispersive and real) is placed between them, at distance $d_{1}$ from body 1 . The system is depicted in Fig. 16 .

The heat flux is evaluated after linearizing the general expressions with respect to the particle polarizability, assuming that the scattering contribution is weak. As a result, the heat flux $\Phi$ (power $\mathcal{P}$ per unit area) is conveniently expresses as

$$
\Phi=\Phi_{\mathrm{vac}}+\Delta \Phi,
$$

where $\Phi_{\text {vac }}$ is the well-known heat flux between two slabs separated by a vacuum gap, and the correction term $\Delta \Phi$ (proportional to $\alpha$ in the linearized approximation) is a direct description of the non-additivity of radiative heat flux.

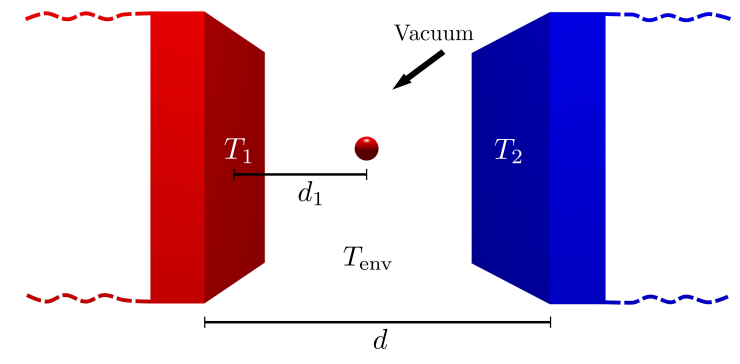

FIG. 16 Two planar slabs (bodies 1 and 2) are placed at distance $d$ and separated by vacuum. A particle of polarizability $\alpha$ is placed at distance $d_{1}$ from slab 1 . From (Müller et al. 2017).
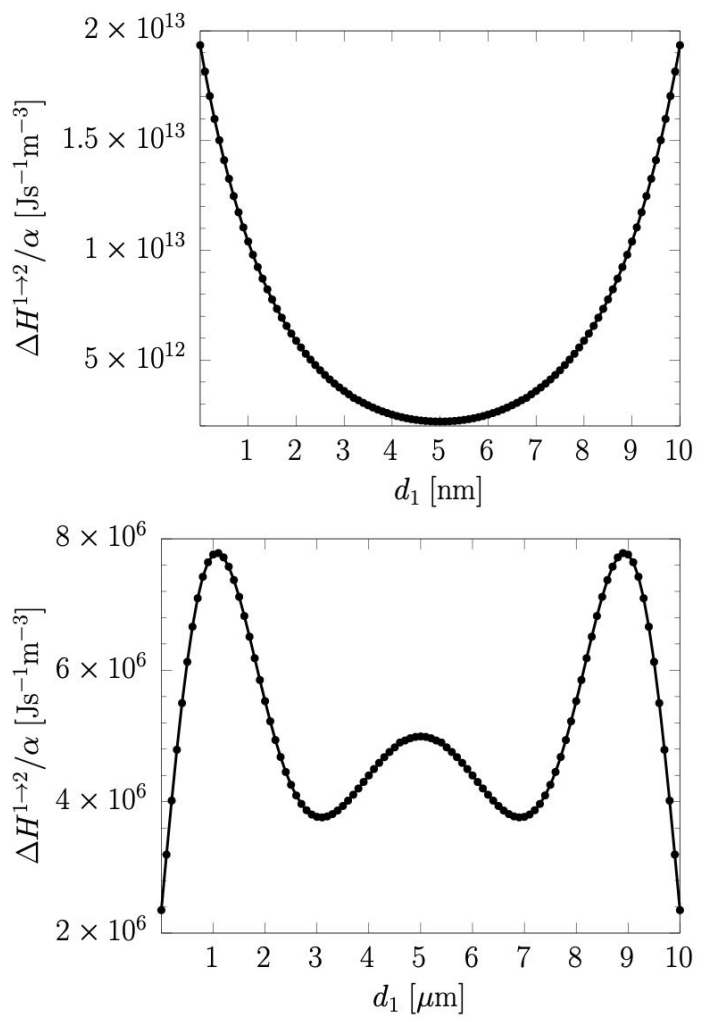

FIG. 17 Non-additive correction to the two-body heat flux $\Delta H^{1 \rightarrow 2}=\Delta \Phi[$ see Eq. [81] ] in the presence of a particle of polarizability $\alpha$. The upper curve corresponds to the nearfield configuration $d=10 \mathrm{~nm}$, while the lower one corresponds to the far field $(d=10 \mu \mathrm{m})$. From (Müller et al. 2017).

The non-additive correction is numerically evaluated for slab temperatures of $301 \mathrm{~K}$ and $300 \mathrm{~K}$ in two difference configurations: for a slab-slab distance $d=10 \mathrm{~nm}$ (near field) and for $d=10 \mu \mathrm{m}$ (far field), as a function of the particle position $d_{1}$ (see Fig. 16). The results are shown in Fig. 17. In both configurations we clearly observe the expected symmetry with respect to the central particle position $d_{1}=\frac{d}{2}$. In the near field, we observe that the effect is maximized when the atom is close to 


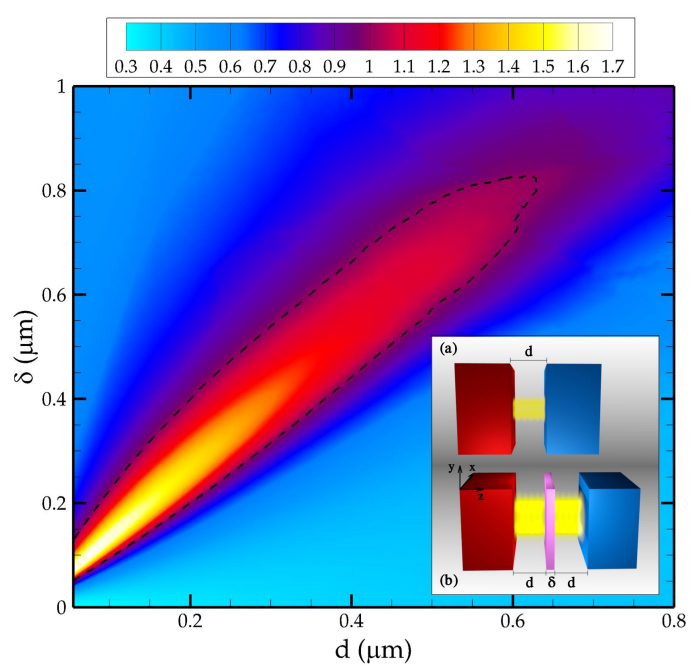

FIG. 18 Heat-flux amplification $\Phi_{3 \mathrm{~s}}(d, \delta) / \Phi_{2 \mathrm{~s}}(d)$ in a threebody configuration compared to a two-body configuration shown in inset as a function of distance $d$ and thickness of the intermediate slab $\delta$. The black dashed line corresponds to the constant value $\Phi_{3 \mathrm{~s}}(d, \delta) / \Phi_{2 \mathrm{~s}}(d)=1$. From (Messina et al. 2012).

one of the two slabs. This reflects, apart from the symmetry of the system, the typical exponentially decreasing behavior of heat flux in the near field, which is in turn a consequence of the dominating contribution of evanescent waves. The situation is clearly different in the far field. First, not surprisingly, the effect is several orders of magnitude smaller that in the near field, Moreover, the external positions $d=0, d_{1}$ are now minima of the effect, which oscillates with respect to $d_{1}$. These oscillations are due to the interferences between propagating waves (dominating in this scenario), reflected between the two plates and scattered by the particle inside the cavity which change the local density of states (Dorofeyev et al. 2002, Francoeur et al. 2010b) at the particle's position which is also known from the context of spontaneous emission of atoms and molecules within a such a configuration (Danz et al. 2002).

Another interesting consequence of three-body effects in NFRHT was shown in Ref. (Messina et al., 2012, Zheng and Xuan, 2011). In these works, the authors considered a system made of three parallel slabs as shown in the inset of Fig. 18. The intermediate slab, of thickness $\delta$, is placed at distance $d$ from the external slabs, assumed to have infinite thickness. This configuration is compared to the standard two-body scenario, shown in the inset of Fig. 18, where the intermediate slab is removed and $d$ is now the distance between the external slabs. We stress that in both systems the minimum distance between adjacent slabs, very relevant parameter in a near-field configuration, is the same. Moreover, for a chosen couple of temperatures (more specifically, 400 and $300 \mathrm{~K}$ ), the temperature of the intermediate slab is taken as the equilibrium one, i.e. the one at which the net flux on it vanishes. Based on this assumption, adding the third intermediate slab has no impact on the energy balance of the system, and thus the third body is only acting as a passive relay added to the two-body system. The heat flux amplification, defined as the ratio $\Phi_{3 \mathrm{~s}}(d, \delta) / \Phi_{2 \mathrm{~s}}(d)$ between the three- and two-body fluxes, is shown in Fig. 18. The figure clearly shows that the flux can be amplified for reasonable values (hundreds of nanometers) of both $d$ and $\delta$, and that this amplification factor goes up to a maximum value around $70 \%$ for small distances. This amplification for $d \approx \delta$ reminiscent of the superlens effect (Biehs et al., 2016 , Pendry, 2000) which leads to an optimal energy transfer between two atoms which are separated by a superlens if the distance $d$ to the interface of the superlens coincides with the thickness of the superlens $\delta$. Here, it is a purely three-body effect, which is confirmed by the spectral and mode analysis performed in Ref. (Messina et al., 2012). More recently patterned intermediate media (Kan et al., 2019), twodimensional atomic systems (Simchi, 2017) and hyperbolic media (Song et al., 2018) have also been considered to enhance furthermore the transfers. The use of such kind of three-body control of heat flux was proposed to design many-body heat engines (Latella et al., 2015) with thermodynamic performances better than their two body counterpart and the thermal analog of transistor (BenAbdallah and Biehs, 2014) driven by photons. In the proposed scheme, the combination of many-body effects and the presence of a phase-change material playing the role of the gate/basis of the transistor, allows to switch, amplify and modulate the heat flux between source/emitter and drain/collector (see also Fig. 32).

It is interesting to remark that the role of a third thermally interacting body can also be played by a thermal bath, described as a body far from the rest of the system and emitting as a black-body surface at a given temperature. This was recently shown in (Latella et al. 2020), where the heat flux between two planar slabs or between a slab and a particle was considered in the presence of a thermal bath. It was shown that, in virtue of manybody interactions taking place in these three-body systems, the flux exchanged between the two slabs (or the slab and the particle) saturates to a constant value when the distance goes to zero even at relatively large separation distance where the non-local optical effects are negligible, as shown e.g. in Fig. 19 in the case of two SiC slabs.

\section{Steady-state temperatures and multistable states}

In arbitrary many-body systems consisting of $N$ objects at temperatures $T_{1}, \ldots, T_{N}$ the time evolution reads $(i=1, \ldots, N)$

$$
I_{i} \frac{\mathrm{d} T_{i}}{\mathrm{~d} t}=\mathcal{P}_{i}\left(T_{1}, . ., T_{N} ; t\right),
$$




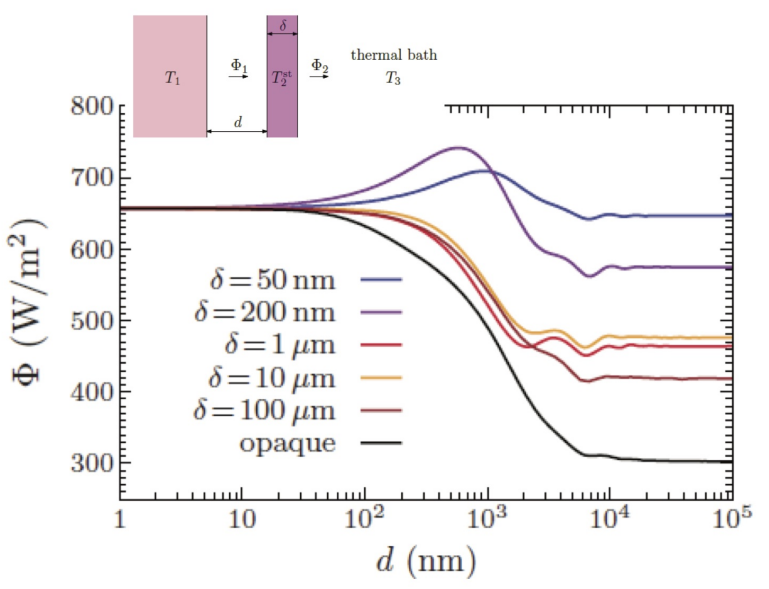

FIG. 19 Heat flux exchanged between two slabs immersed in a thermal bath with respect to their separation distance $d$. Slab 1 has a fixed temperature of $T_{1}=400 \mathrm{~K}$, while the thermal bath is at $T_{3}=300 \mathrm{~K}$. The second slab, of thickness $\delta$, thermalizes to the equilibrium temperature at which the net flux it receives vanishes. From (Latella et al. 2020).

where $I_{1}=\rho_{i} C_{i} V_{i}$ is the termal inertia defined by the heat capacity $C_{i}$, volume $V_{i}$, and the mass densite $\rho_{i}$ of the $i^{t h}$ object while $\mathcal{P}_{i}$ is the net power received by this object. Following expressions in Eq. (72) and (56) the latter can be broken up into

$$
\mathcal{P}_{i}\left(T_{1}, . ., T_{N} ; t\right)=\sum_{j \neq i} \mathcal{P}_{i j}\left(T_{1}, . ., T_{N} ; t\right)+\mathcal{P}_{i b}(t)
$$

where $\mathcal{P}_{i j}$ is the power exchanged between the $j^{t h}$ and $i^{\text {th }}$ object and $\mathcal{P}_{i b}$ is the power exchanged between object $i$ and the background which can also be an external heat bath or thermostat connected to object $i$. If all $\mathcal{P}_{i}$ are linear functions of the temperatures which is generally the case close to the global equilibrium or nonequilibrium steady state (in the following, for the sake of notation simplicity, we use the abreviation $T^{\text {eq }}$ for the steady-state temperatures), i.e. for small temperature differences $\left.\left|T_{i}-T_{j}\right| \ll \min \left(T_{1}, \ldots, T_{N}\right)\right)$, the system of equations can be linearized by introducing the conductances

$$
G_{i j}=\frac{\partial \mathcal{P}_{i j}}{\partial T_{j}}
$$

as done in Eq. (93). For multilayer systems with infinitely large interfaces the above equations can be used as well by simply replacing the quantities by the corresponding quantities normalized to a surface area $A$ so that the thermal inertia becomes the thermal inertial per area $I_{i} \rightarrow I_{i} / A$, the dissipated power becomes the heat flux $\mathcal{P}_{i} \rightarrow \mathcal{P}_{i} / A \equiv \Phi_{i}$, and the conductance becomes the heat transfer coefficient $G_{i j} \rightarrow G_{i j} / A \equiv H_{i j}$.

When assuming that no energy is added or removed from outside of the system, the thermal steady state is a solution of the system of equations $(i=1, \ldots, N)$

$$
\mathcal{P}_{i}\left(T_{1}, . ., T_{N}\right)=0, .
$$

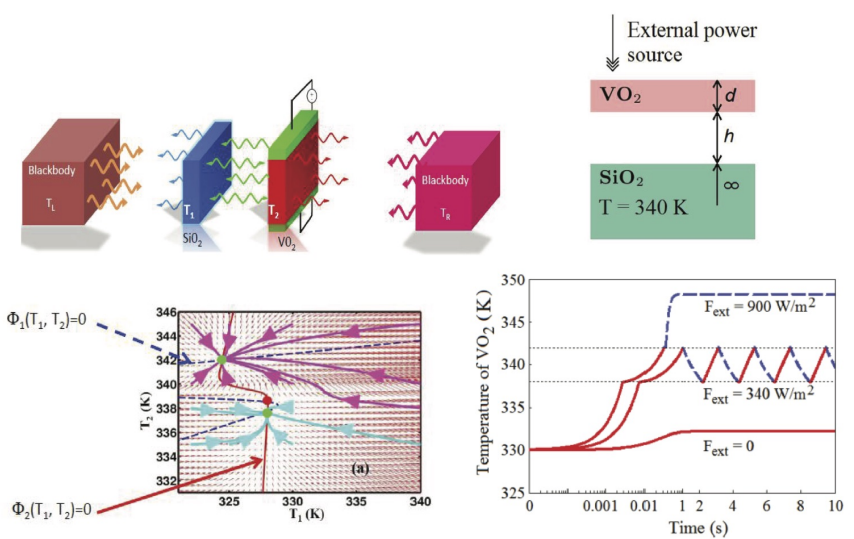

(a)

(b)

FIG. 20 (a) Phase portrait (i.e. trajectories of temperatures) in a bistable system consisting of two membranes of $\mathrm{SiO}_{2}$ and $\mathrm{VO}_{2}$ in interaction with two thermal baths for different initial conditions. The green (red) points denote the stable (unstable) global steady-state temperatures. From (Kubytskyi et al. 2014). (b) Self-oscillation of the temperature of a $\mathrm{VO}_{2}$ membrane in vicinity of a $\mathrm{SiO}_{2}$ substrate when adding a specific external constant power $F_{\text {ext }}$. From Dyakov et al. 2015a)

The local thermal equilibrium of the $i^{\text {th }}$ object is reached when $\mathcal{P}_{i}\left(T_{1}, . ., T_{N}\right)=0$. This equation defines a hypersurface in temperature space. The intersection of the hypersurfaces associated to all local equilibria defines the global steady state of the system. In the specific case where the system is composed of two objects the local equilibrium state of each object corresponds to a curve in the two dimensional space of temperatures $\left(T_{1}, T_{2}\right)$ and the intersection of the two local equilibrium lines defines the global steady-state temperatures.

If all $\mathcal{P}_{i}$ are linear functions of the temperatures which is generally the case close to the global equilibrium or steady state and when the conductances $G_{i j}$ can be considered as independent of the temperatures, i.e. when in particular the material properties can be considered as temperature independent, the system has a unique solution $\left(T_{1}^{\mathrm{eq}}, \ldots, T_{N}^{\mathrm{eq}}\right)^{t}$. On the contrary, when the optical properties of materials are temperature dependent $\mathcal{P}_{i}$ become nonlinear with respect to the temperatures. In this case, the system of equations (85) might admit more than one steady-state solution. Among these temperature solutions one finds in general stable and unstable solutions. The stability of these temperatures can be assessed by following a perturbative approach. Starting from a steady state $\alpha$ with temperature $\left(T_{1, \alpha}^{\mathrm{eq}}, \ldots, T_{N, \alpha}^{\mathrm{eq}}\right)^{t}$ and adding a small perturbation then the dynamics is described by the 

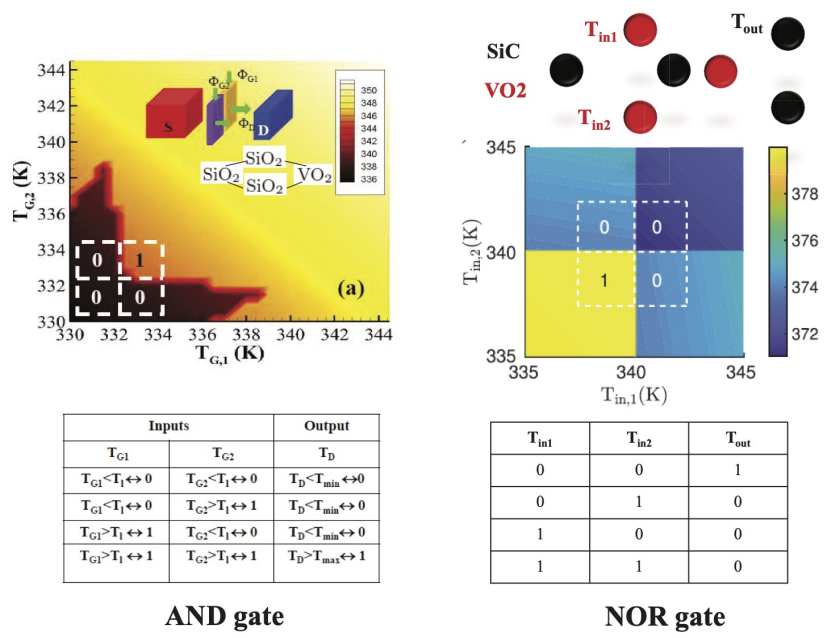

\begin{tabular}{|c|c|c|}
\hline $\mathbf{T}_{\text {in1 }}$ & $\mathbf{T}_{\text {in2 }}$ & $\mathbf{T}_{\text {out }}$ \\
\hline 0 & 0 & 1 \\
\hline 0 & 1 & 0 \\
\hline 1 & 0 & 0 \\
\hline 1 & 1 & 0 \\
\hline \multicolumn{3}{|c|}{ NOR gate }
\end{tabular}

FIG. 21 (left) AND gate made with two $\mathrm{SiO}_{2}$ membranes (gates) suspended between a thermal $\mathrm{SiO}_{2}$ source and a $\mathrm{VO}_{2}$ drain. The color map represents the output temperature $T_{D}$ of the drain with respect to the two input temperatures $T_{G 1}$ and $T_{G 2}$ of the two gates. In the bottom is the thruth table for the AND gate. From (Ben-Abdallah and Biehs, 2016). (right) $\mathrm{NOR}$ gate designed by coupling of $\mathrm{SiC}$ and $\mathrm{VO}_{2}$ nanoparticles. From (Kathmann et al. 2020)

following linearized system

$$
\frac{\mathrm{d}}{\mathrm{d} t}\left(\begin{array}{c}
\delta T_{1, \alpha}(t) \\
\vdots \\
\delta T_{N, \alpha}(t)
\end{array}\right)=\mathrm{J}\left(\begin{array}{c}
\delta T_{1, \alpha}(t) \\
\vdots \\
\delta T_{N, \alpha}(t)
\end{array}\right)
$$

where $\delta T_{i, \alpha}(t)=T_{i}-T_{i, \alpha}^{\mathrm{eq}}(i=1, \ldots, N)$ is the perturbation from the steady state $\alpha$ and

$$
\mathrm{J}=\left(\begin{array}{ccc}
\frac{\partial \mathcal{P}_{1}}{\partial T_{1}} & \cdots & \frac{\partial \mathcal{P}_{1}}{\partial T_{N}} \\
\vdots & & \vdots \\
\frac{\partial \mathcal{P}_{N}}{\partial T_{1}} & \cdots & \frac{\partial \mathcal{P}_{N}}{\partial T_{N}}
\end{array}\right)
$$

is the Jacobian matrix associated to the dynamical system (82). As in any linear dynamical system the sign of the eigenvalues of $\mathbb{J}$ allows us to conclude on the stability of thermal state.

The demonstration of multistable thermal behaviors in many-body systems as shown in Fig. 20 has opened the possibility to design thermal analogs of volatile electronic memories (Ben-Abdallah and Biehs, 2015, 2017; Dyakov et al., 2015b: Khandekar and Rodriguez, 2017; Kubytskyi et al. 2014), logic gates (Ben-Abdallah and Biehs, 2016. Kathmann et al., 2020) (see Fig. 21) and self-oscillating systems (Dyakov et al. 2015a) that allow to switch from one global equilibrium to another and which can be potentially interesting for practical realization of heat engines (Latella et al. 2015, 2014).

\section{Radiative heat transfer in reciprocal many-body systems}

In the 2000s the first attempts of treating heat transfer in $N$-body systemss were made in order to quantify the contribution of plasmonic modes to the thermal conductance in one dimensional arrays of nanoparticles in nanofluids (Ben-Abdallah, 2006, Ben-Abdallah et al. 2008). Inside these simple networks all inner nanoparticles are assumed to be at zero temperature while the two particles at both ends of the chain are connected to two thermostats. In these systems heat carried by photons is simply scattered between the two thermostats. But in contrast to Polder and Van HoveâĂŹs theoretical framework, which is based on the FE theory, in these works a kinetic approach has been followed. The main features and limitations of this approach will be discussed in the next section.

\section{Kinetic approach vs exact calculations}

This approximate theory is based on the solution of a Boltzmann transport equation

$$
\frac{\partial f}{\partial t}+v_{g}(k) \frac{\partial f}{\partial z}=\left[\frac{\partial f}{\partial t}\right]_{\text {coll }} .
$$

for the distribution function $f$ of thermal photons inside a given system. Here $v_{g}(k)$ is the group velocity of the mode $k$ and the rhs of this equation stands for the collission term which can be simplified within the relaxation time approximation. When assuming that one thermostat is at temperature $T$ and the other one at zero temperature, then the power $\mathcal{P}$ flowing through this system results from the calculation of first-order moment associated with the photonic equilibrium distribution function $f=n(\omega, T)$ (Ben-Abdallah et al. 2008)

$$
\mathcal{P}=\sum_{\ell=1}^{\infty} \int_{0}^{\infty} \frac{\mathrm{d} k}{2 \pi} \hbar \omega_{\ell}(k) v_{g, \ell}(k) n\left(\omega_{\ell}(k), T\right),
$$

where $\omega_{\ell}(k)$ is the dispersion relation of resonant multipole modes $\ell$ supported by the structure. The conductance is then defined as $G=\partial \mathcal{P} / \partial T$. It is important to note that only the eigenstates of the system are assumed to play a role in the heat transport process. Since these preliminary studies, more complex systems like chains of ellipsoidal polaritonic particles (OrdonezMiranda et al., 2015), nanoparticle crystals (OrdonezMiranda et al., 2016; Tervo et al., 2016), nanoresonators inclusions (Tervo et al., 2019b) or chains of graphene disks (Ramirez and McGaughey, 2017) have been investigated (see Fig. 23) as well as multilayer photonic crystals (Lau et al., 2009, 2008) using this kinetic approach. But as shown recently within a full FE calculation based on the $N$-body theory introduced in Sec. III.A.2, the kinetic approach fails in describing heat exchanges in systems where heat is also carried by non-resonant modes over a broad spectral band (Kathmann et al. 2018). 

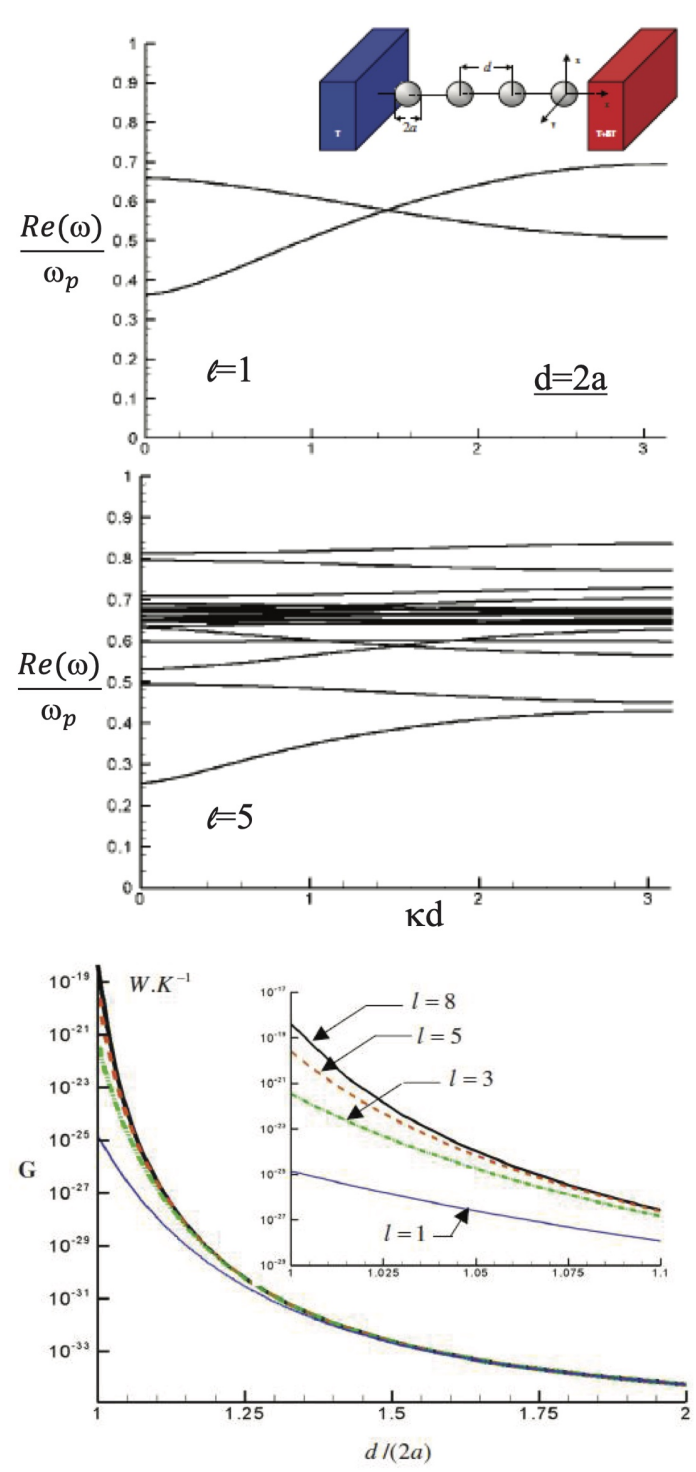

FIG. 22 (a) Dispersion curves (real part) of collective plasmonic modes along a chain of copper nanoparticles dispersed in vacuum in the case of dipolar moments $(\ell=1)$ and for the multipolar moments of order $\ell=5$. (b) Thermal conductance $G$ of linear chains of copper particles calculated from the kinetic theory for different multipole orders $\ell$ versus the separation distance $d$ normalized to the particle diameter $2 a$. The inset is a zoom on the near-contact region. From (BenAbdallah et al., 2008).

This result has been confirmed recently ( Tervo et al. 2020)). Further studies of conductance within two and three dimensional dipolar systems based on the fluctational electrodynamic calculations have been published recently (Tervo et al., 2019a) which opens also to test the validity of the kinetic approach in such systems as studied in (Ordonez-Miranda et al., 2016, Tervo et al., 2016). A discussion of the conductance within multilayer photonic crystals within the $\mathrm{FE}$ approach discussing the role of surface phonon polaritons can be found in
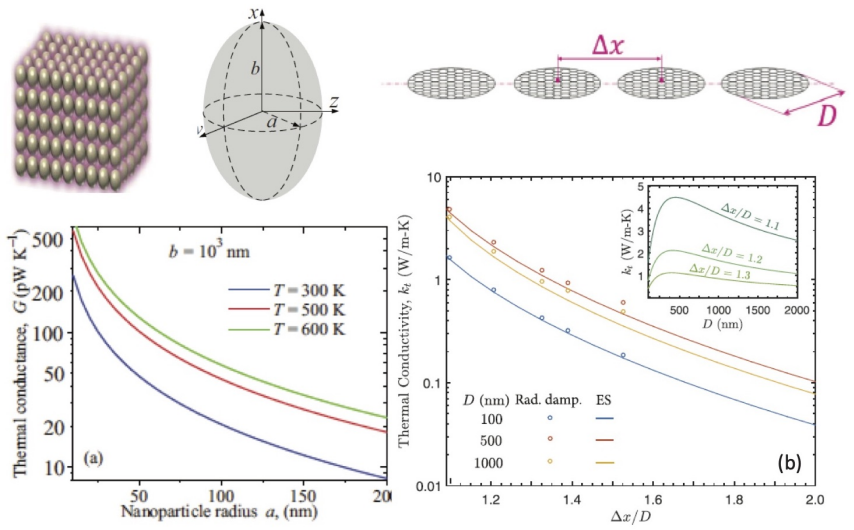

FIG. 23 (a) Thermal conductance of the colloidal crystals made up of spheroidal SiC nanoparticles, as a function of their horizontal radius. (b) Thermal conductivity of coplanar disk arrays for different diameters and separations at temperature $T=300 \mathrm{~K}$. From (Ordonez-Miranda et al. 2016, Ramirez and McGaughey, 2017).

(Narayanaswamy and Chen, 2005: Tschikin et al., 2012a).

\section{Heat transfer in complex networks}

Based on the rigorous $\mathrm{FE}$ approach we will now address the heat flux in arbitrary systems. The thermal behavior of fractal structures and the heat exchanges between fractal clusters of nanoparticles has also been theoretically investigated. These studies have revealed (Dong et al. 2017b; Nikbakht, 2017) that the (self)conductance increases as $R^{\overline{D_{f}}}$ where $R$ is the gyration radius of the structure and $D_{f}$ its fractal dimension (see Fig. 24(a)). When two of these structures interact in near-field the thermal conductance of heat exchange between metallic clusters increases with the fractal dimension as can be seen in Fig. 24(b). Moreover, in contrast to ordered media, the localization of plasmons or phonon-polaritons in fractal structures could be responsible of a significant reduction of the self-conductance in fractal structures although no clear evidence about this claim has been presented so far. However, a recent study (Luo et al. 2019) has revealed that the heat transfer between fractal structures does not depend on their fractality at separation distance larger than the localization lengths, which tends to confirm this statement.

Beside their original thermal properties several physical effect inherent to many-body systems have been highlighted in complex plasmonic structures. Among these effects, a thermal analog of Coulomb drag effect in nanoparticle networks has been recently predicted theoretically (Ben-Abdallah, 2019a). The configuration is sketched in Fig.25. As in its electric counterpart where interactions at close separation distances (compared to the range of Coulombic interactions) of free charge carriers between two electric conductors gives rise to a drag current in a passive conductor when a bias voltage is 


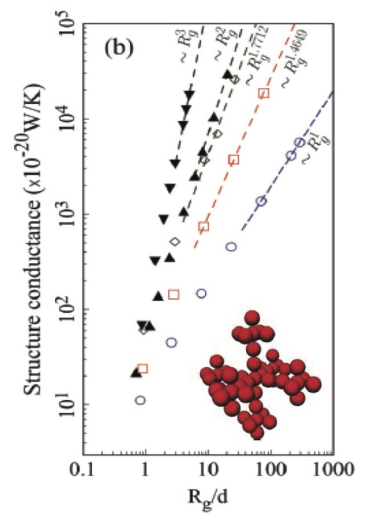

(a)

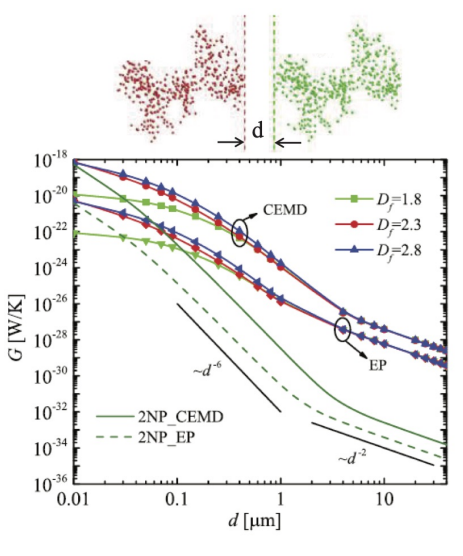

(b)
FIG. 24 (a) Thermal conductance of Vicsek fractal structures as a function of normalized gyration radius. From (Nikbakht, 2017) (b) Thermal conductance between two Ag nanoparticles clusters at various fractal dimensions. From (Luo et al. 2019)

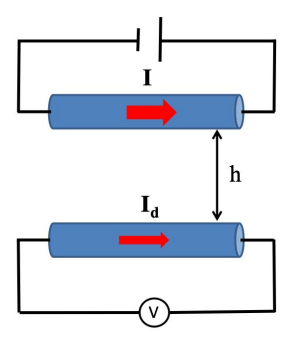

(a)

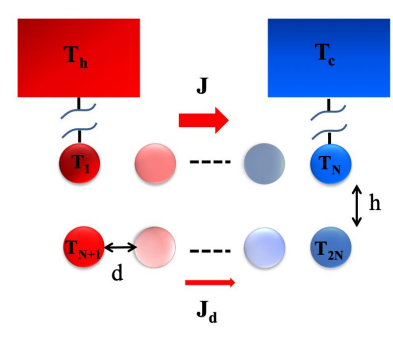

(b)
FIG. 25 (a) Illustration of the classical Coulomb drag effect. A drag electric current $I_{d}$ in a passive conducting wire is induced by a primary current $I$ flowing in a driving conductor placed close to it. (b) Radiative drag effect in a many-nody system: a drag heat flux $J_{d}$ carried by thermal photons between two particles is induced by a heat flux $J$ exchanged between two thermostated objects in a many-body system. From (Ben-Abdallah, 2019a).

applied along the so called drive conductor, a radiative heat flux in a many-body systems can be induced in a given region by a primary flux generated by a temperature gradient in another region of the system. In the case of two parallel chains of nanoparticles as sketched in Fig 25(b), where the extremities of the first chain are held at fixed temperature with two external thermostats while all other particles can relax to their own local equilibrium temperature, the magnitude and the direction of drag flux can be calculated using the following procedure.

In the steady state the net power received by each particle vanishes which allows to determine unknown temperatures $\left(T_{2}, . ., T_{N-1}, T_{N+1}, \ldots, T_{2 N}\right)\left(T_{1}\right.$ and $T_{N}$ are fixed by the thermostats) and the power $\mathcal{P}_{1}$ and $\mathcal{P}_{N}$ coming from the external thermostats in order to keep the temperatures of particle 1 and $N$ fixed. Then the heat current in the upper chain in Fig. 25(b)

$$
J=\mathcal{P}_{N}-\mathcal{P}_{1}
$$

as well as the induced heat current in the lower chain in Fig. 25(b)

$$
J_{D}=\mathcal{P}_{2 N}-\mathcal{P}_{N+1}
$$

can be determined. Finally, the thermal drag resistance

$$
R_{D}=\frac{T_{N+1}-T_{2 N}}{J} .
$$

quantifies the frictional effect induced by the electromagnetic interactions between the different regions inside the system. In hybrid polar-metal systems this friction can be negative (Ben-Abdallah, 2019a) proving the existence of regions within these systems where heat can locally flow in an opposite direction to the applied temperature gradient.

Beside this generation of heat flux by frictional effect in many-body systems the temperature of the particles in particle networks can be individually addressed with a subwavelength accuracy (Yannopapas and Vitanov, 2013) using external excitations such as chirped pulses and can be controlled by adaptive optimization techniques at the time scale of thermal relaxation processes. The interplay between nano-objects can also be used to focus and even pump heat (Ben-Abdallah, 2019b) outside of the system itself. The heat flux radiated through an oriented surface by a collection of emitters held at different temperature $T_{i}(i=1, \ldots, N)$ can be calculated from Eq. (59). By tuning the temperature of three thermal emitters in vicinity of a substrate as shown in Fig 26. for instance, the heat flux can be locally focused and even amplified in tiny regions which are much smaller than the diffraction limit and even smaller than the regions heated with a single emitter (Ben-Abdallah, 2019b). This control of flux lines by a collection of nano-sources can be used to tailor the heat flux at the nanoscale or to analyze and change at this scale the local temperature of solid surfaces.

\section{Long range heat transport and amplification of heat flux}

Instead of enhancing the heat flux between two nanoparticles or two slabs by introducing an intermediate nanoparticle or slab as discussed in Sec. III.A.3 and III.B.2 it is also possible to guide the radiative heat flux over a long distance by exploiting the properties of specific modes such as surface or hyperbolic modes supported by some structures. This guiding can for example be done by bringing two nanoparticles close to a planar interface as sketched in Fig 27(a) which supports a surface polariton in the infrared. Then the hot nanoparticle 
(a)

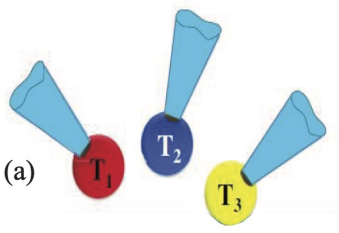

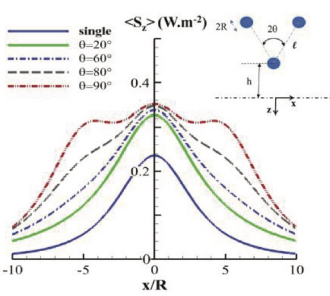

(b)

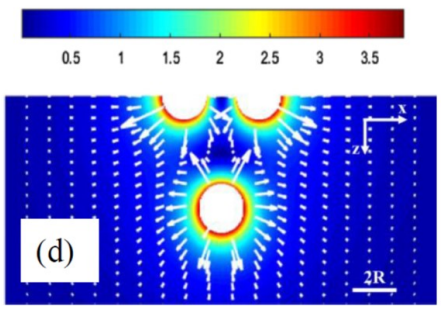

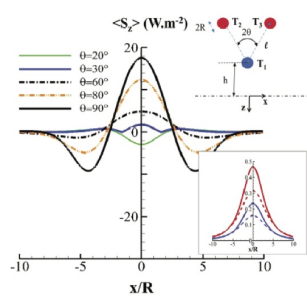

(c)

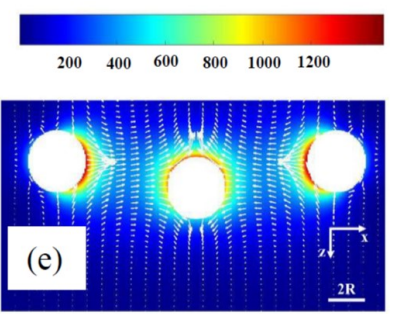

FIG. 26 (a) Schematic of a multi-tip SThM platform with three tips. Nano-spheres (thermal emitters) are grafted on single scanning probe tips and held close to a substrate. Their temperatures and positions are individually controlled. (b) Normal component $\left\langle S_{z}\right\rangle$ of the Poynting vector radiated through the substrate surface at $z=0$ by a three-tip SThM setup with glass nano-emitters at $T=300 \mathrm{~K}$. (c) As in (b) but with $T_{2}=T_{3}=350 \mathrm{~K}$ (red) and $T_{1}=300 \mathrm{~K}$ (blue). The inset shows the flux at $z=0$ for a single particle at $T=300 \mathrm{~K}$. (d)-(e) Magnitude of Poynting vector field in the $(x, z)$ plane radiated by a multi-tip setup in the case (d) for an angular opening of $\theta=20^{\circ}$ and in the case (e) for an angular opening $\theta=80^{\circ}$. From (Ben-Abdallah, 2019b).

can directly couple to this surface mode and subsequently transfer its heat to the second (cold) particle over relatively long distances.

Such a transport has been first investigated in Ref. (Sääskilahti et al. 2014) between polar nanoparticles above single polaritonic surfaces and inside cavities formed of two mirrors or made with slabs supporting surface modes. This study and more recent studies (Asheichyk and Krüger, 2018, Dong et al., 2018, Messina et al., 2018) have shown that the heat current between dipoles placed in a cavity can be enhanced by several orders of magnitude as compared to the free-space heat current with a similar interparticle distance. In particular, in Ref. (Messina et al. 2018) it has been shown that a similar enhancement and long range heat transport can be also observed between metallic particles when a graphene sheet cover a $\mathrm{SiC}$ interface. In this case the heat-flux can be enhanced by several orders of magnitude at interparticle distance of about $1-10 \mu \mathrm{m}$ as shown in Fig. 27(b) (a)
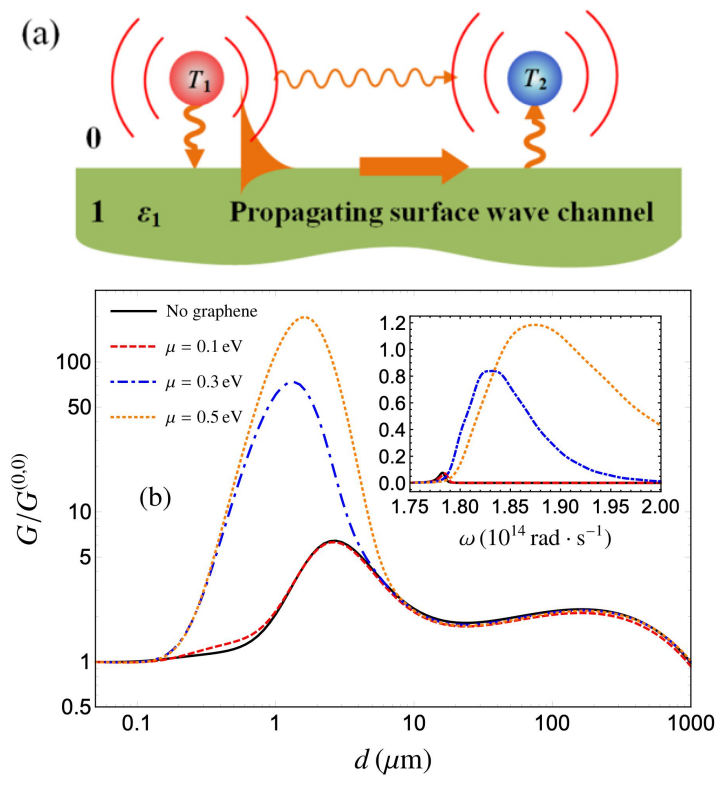

FIG. 27 (a) Heat flux between two nanoparticles at interparticle distance $d$ by coupling via the surfacce modes of an inferface. From Ref. (Dong et al., 2018). (b) conductance ratio $G / G^{(0,0)}$ ( $G$ conductance with interface and $G^{(0,0)}$ without interface) as a function of $d$ between two Au nanoparticles placed at distance $z=150 \mathrm{~nm}$ from a SiC substrate. The four lines correspond to the absence of graphene (black solid line), and to configurations with graphene having $\mu=0.1 \mathrm{eV}$ (red dashed line), $0.3 \mathrm{eV}$ (blue dot-dashed line) and $0.5 \mathrm{eV}$ (orange dotted line). The inset shows the spectral conductance associated with the four same configurations. From Ref. (Messina et al. 2018).

suggesting that the near-field enhanced thermal radiation can be brought to distances which are comparable to the thermal wavelength. Similar enhancement effects were reported for the heat flux along chains of nanoparticles close to a phonon-polaritonic interface (Dong et al. 2018), between two nanoparticles mediated by an intermediate macroscopic phonon polaritonic sphere Asheichyk et al. 2017), by an anisotropic meta-surface made of graphene stripes (Zhang et al., 2019a) or a stack of graphene sheets (He et al. 2019b). As shown in (Ott and Biehs, 2020) the distance at which the maximum heat flux enhancement occurs is connected to the propagation length of surface modes (Ott and Biehs, 2020). Hence, the enhancement mechanism for the heat flux is reminiscent of the enhancement of Förster resonance energy transfer between atoms, molecules, or quantum dots which are brought in close vicinity to a plasmonic interface where also a maximal enhancement is found at distances coinciding with the propagation length of the surface modes involved in the energy transport (Biehs and Agarwal, 2013b; Bouchet et al., 2016, Poudel et al. 2016; Velizhanin and Shahbazyan, 2012) allowing for a long-range energy transfer.

Motivated by the very promising properties of hyper- 
bolic metamaterials for long-range Förster energy transfer (Biehs et al., 2016, Deshmukh et al., 2018; Newman et al. 2018) another strategy has been explored to transport the near-field heat flux over long distances using such hyperbolic guides. Hence it has been shown that the large wavevector surface waves supported by polaritonic materials can be converted into propagating hyperbolic modes inside these media so that the usual ultrasmall penetration depth of near-field heat flux (Basu and Zhang, 2009) can become very large (Biehs and BenAbdallah, 2017, Lang et al., 2015, Tschikin et al., 2015) in these guides as well as the amount of heat they can transport (Biehs et al., 2015, Liu and Narimanov, 2015). Since the hyperbolic media can support hyperbolic modes over a broad spectral band the flux they can transport can be very high. It seems even possible to achieve with hyperbolic metamaterials a radiative thermal conductivity which can in principle be comparable to the phononic conductivity (Biehs et al. 2015; Liu and Narimanov, 2015). Recently, first experimental steps have been made to verify this claim (Salihoglu et al. 2019), but the experimental results are not yet convincing. In a more detailed study it could be demonstrated that the near-field heat flux between two slabs can be guided through a hyperbolic waveguide over distances larger than the thermal wavelength so that larger heat fluxes than the blackbody value are achievable for far-field distances (Messina et al. 2016a). On the other hand, it could also be shown that the guiding performance highly depends on the dissipative properties of the waveguide material and that for long-distance guiding also low-loss infrared materials like Ge, for instance, would already have very good longrange guiding properties (Messina et al. 2016a). The long-range guiding effect has also been verified for the heat flux between two nano-particles through a hyperbolic multilayer structures (Zhang et al., 2019b) as shown in Fig. 28 .

Even though the enhancement of the heat flux due to coupling to the surface modes of the phonon-polaritonic or plasmonic structures can be several orders of magnitude it has to be kept in mind that the mentioned studies consider the steady-state heat flux between the nanoparticles and that the enhancement is relativ to the case where the interface is removed. Hence, even by increasing the heat flux by several orders of magnitude at a distance of 1 micron the absolute value of the heat flux is still small, because the heat flux between the nanoparticles follows the $1 / d^{6}$ law in the near-field regime (Volokitin and Persson, 2001). Furthermore, it should be kept in mind that by bringing the nanoparticles in close vicinity of an interface not only the heat flux between the particles increases, but also the thermal emission of the hot particle into the substrate so that the hot particle will rather tend to cool by thermal emission into the substrate then by heating the cooler nanoparticle. However, a first thermal relaxation study shows (Ott and Biehs, 2020) that by choosing wisely the distances between the nanoparticles and between the nanoparticles and the interface, a
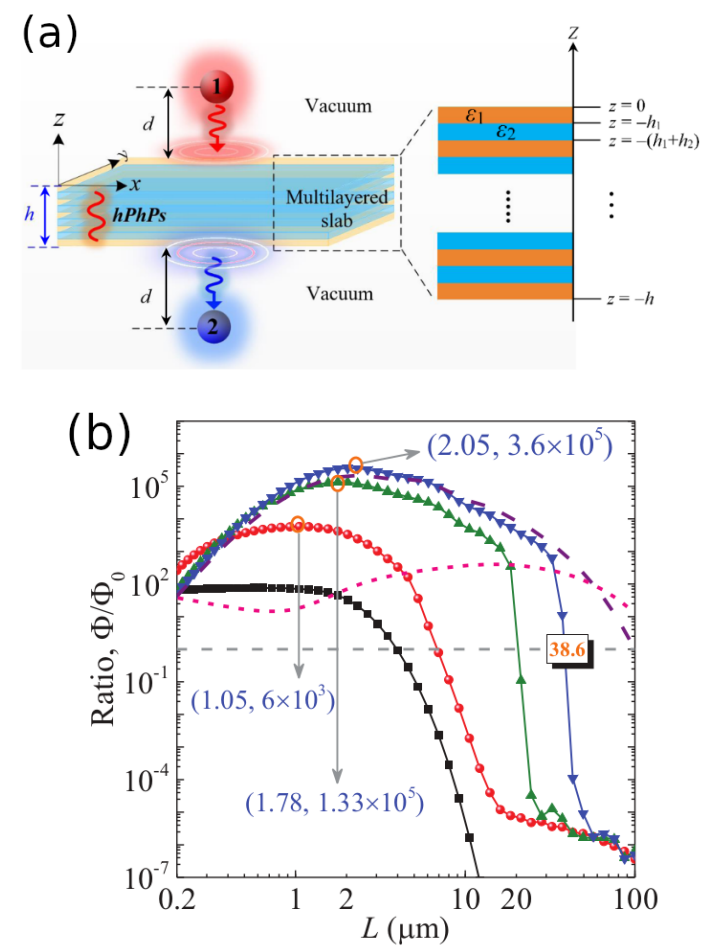

FIG. 28 (a) Sketch of heat flux between two nanoparticles through a hyperbolic multilayer meta-material. (b) Exchanged power $\Phi$ as function of interparticle distance $L$ normalized to the exchanged power $\Phi_{0}$ where the hyperbolic multilayer meta-material has been replaced by vacuum. From Ref. (Zhang et al. 2019b).

substantial heating of the cold nanoparticle can be observed. Similar considerations also hold for the heat flux though a structure. Hence, it is very useful to focus in future studies on heat fluxes and the thermal relaxation or actual heating/cooling performance as well.

\section{Relaxation dynamics}

The temporal dynamics of any many-body system in interaction with an external environment or with local thermostats is simply driven by the competition between its thermal inertia and the strength of the thermal link with the external environment and these thermostats. Close to the thermal equilibrium, the time evolution of temperatures $\mathbf{T}=\left(T_{1}, \ldots, T_{N}\right)$ in Eq. 83 is driven by the linear dynamical system

$$
\mathrm{I} \frac{\mathrm{d} \mathbf{T}}{\mathrm{d} t}=-\mathbb{C} \mathbf{T}(t)+\mathbb{C}_{b} \mathbf{T}_{b}
$$

where $\mathbb{I}=\operatorname{diag}\left(I_{1}, \ldots, I_{N}\right)$ is the diagonal inertia matrix which depends on the mass density, heat capacity and size of each element, $\mathbf{T}_{b}=\left(T_{b 1}, \ldots, T_{b N}\right)$ is the temperature of external bath and reservoirs with which each elements interact, $\mathbb{C}_{b}=\operatorname{diag}\left(G_{1 b}, \ldots, G_{N b}\right), G_{i b}$ being the thermal conductance between the $i^{\text {th }}$ element and the 


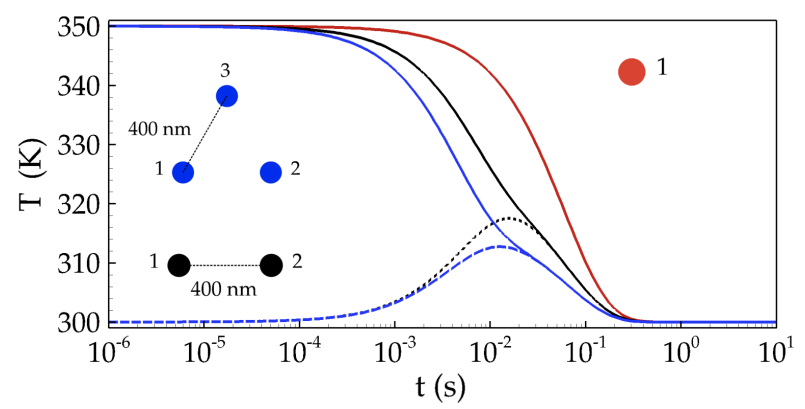

FIG. 29 Time evolution of thermal state in a single (red), two-body (black) and a three-body system (blue) in a bath at temperature $T=300 \mathrm{~K}$. The distance between particles 1 and 2 is $400 \mathrm{~nm}$, while the distances (solid line for dipole 1 , dashed line for dipole 2 , and dot-dashed line for dipole 3 ). From (Messina et al. 2013b).

bath or a thermostat while $\mathbb{C}$ is the general conductance matrix with components

$$
\mathbb{C}_{i j}=\left(\sum_{k \neq i} G_{i k}+G_{i b}\right) \delta_{i j}-\left(1-\delta_{i j}\right) G_{i j}
$$

with $G_{i j}$ the conductance between the $i^{t h}$ and $j^{t h}$ element defined as follows

$$
G_{i j}=\left.3 \int_{0}^{\infty} \frac{\mathrm{d} \omega}{2 \pi} \hbar \omega \frac{\partial n}{\partial T}\right|_{T=T_{j}} \mathcal{T}_{i j}(\omega) .
$$

A corresponding definition can also be used for slabs. Note that this definition is only valid in the absence of temperature dependence of optical properties of the materials involved. When the conductance matrix is independent of time the thermal state of the system reads

$$
\begin{aligned}
\mathbf{T}(t)=\exp \left[-\mathbb{I}^{-1} \mathbb{C} t\right] \mathbf{T}(0) & \\
& +\int_{0}^{t} \exp \left[-\mathbb{I}^{-1} \mathbb{C}(t-\tau)\right] \mathbb{I}^{-1} \mathbb{C}_{b} \mathbf{T}_{b}(\tau) d \tau
\end{aligned}
$$

$\mathbf{T}(0)$ being the initial state. Hence, it is clear that the relaxation dynamic is driven by the set $\left\{\Gamma_{i}\right\}$ of eigenvalues of the matrix $\mathbb{I}^{-1} \mathbb{C}$, the dominant relaxation time is given by $\tau=1 / \min \left(\Gamma_{i}\right)$ ( $\mathbb{C}$ being a strictly diagonally dominant matrix with positive diagonal elements).

Generally speaking the relaxation process takes place at different scales (Messina et al., 2013b). When the separation distance between the different elements is subwavelength they are first thermalized in near-field regime at the same temperature. This generally happens in few millisecond (Wang and $\mathrm{Wu}$, 2016) for objects of nanometric size (Fig 29) and even in hundreds microseconds for two dimensional nanosystems (Zundel and Manjavacas, 2020). In a second step each element and therefore the whole system thermalizes in far-field toward the bath temperature.

This difference in the time scales for the relaxation dynamics can also be studied in a simpler system when considering a single nanoparticle at temperature $T_{1}$ close to a sample with a fixed background temperature $T_{b}$ then the dynamical equation in 93 reduces to

$$
\frac{\mathrm{d} T_{1}}{\mathrm{~d} t}=\frac{G_{1 b}}{I_{1}}\left(T_{b}-T_{1}\right)
$$

or equivalently

$$
\frac{\mathrm{d} \Delta T}{\mathrm{~d} t}=-\Gamma \Delta T
$$

where $I_{1}=\rho C_{\mathrm{p}} V$ is the thermal inertia of the nanoparticle and $\Delta T=T_{1}-T_{b}$ and the relaxation rate $\Gamma=$ $G_{1 b} / I_{1}$. The solution to this differential equation is simply $\Delta T(t)=\Delta T(0) \exp (-\Gamma t)$ or $T_{1}(t)=\left(T_{1}(0)-\right.$ $\left.T_{b}\right) \exp (-\Gamma t)+T_{b}$. Hence, the relaxation time in this case is the inverse of the relaxation rate $\tau=\Gamma^{-1}$ which is itself determined by the thermal inertia and the heat conductance between the nanoparticle and the sample.

The heat conductance for this configuration has been studied for spherical dielectric and metallic nanoparticles close to a sample with a flat surface (Chapuis et al., 2008b; Dedkov and Kyasov, 2007, Dorofeyev, 1998; Mulet et al. 2001; Volokitin and Persson, 2002), between a spherical dielectric nanoparticle and a structured or rough surface (Biehs and Greffet, 2010c, Biehs et al. 2008, Kittel et al., 2008; Rüting et al., 2012) and between dielectric and metallic ellipsoidal particles and a flat or structured surfaces (Biehs et al. 2010, Huth et al. 2010). Here we focus on a spherical nanoparticle with radius $R$ in a distance $d$ over a planar interface. For $d \gg R$ it can be shown Chapuis et al. 2008b; Dedkov and Kyasov, 2007, Dorofeyev, 1998; Mulet et al., 2001; Volokitin and Persson, 2002) that $G_{1 b}$ is proportional to the electric (magnetic) photonic local density of states $D^{\mathrm{E}}(\omega, d)\left(D^{\mathrm{H}}\right)$ as defined in Agarwal, 1975b Eckhardt, 1982 ) for dielectric (magnetic) nanoparticles above a dielectric (magnetic) substrate. Hence, when disregarding mixed cases as considered in (Dong et al., 2017a; Manjavacas and de Abajo, 2012) the relaxation rate can be written as (Tschikin et al. 2012b)

$$
\Gamma=\left.\frac{1}{I_{1}} \sum_{i=E, H} \int_{0}^{\infty} \mathrm{d} \omega 2 \hbar \omega^{2} \operatorname{Im}\left(\alpha^{i}\right) D^{i}(\omega, d) \frac{\mathrm{d} n}{\mathrm{~d} T}\right|_{T_{b}}
$$

where $\alpha^{E}$ is its electric and $\alpha^{H}$ its magnetic polarizability. The latter takes the magnetic moments due to eddy currents into account which play an important role for thermal emission of metallic nanoparticles (Chapuis et al., 2008b, Dedkov and Kyasov, 2007; Martynenko and Ognev, 2005: Tomchuk and Grigorchuk, 2006). Hence, we find that in comparison to the spontaneous emission of an atom or molecule above a substrate (Novotny and Hecht, 2006 ) where the emission rate is proportional to the local density of states for the transition frequency, the thermal emission rate is given by a spectral average of the local density of states with respect to $\hbar \omega \mathrm{d} n / \mathrm{d} T$. Hence, the 

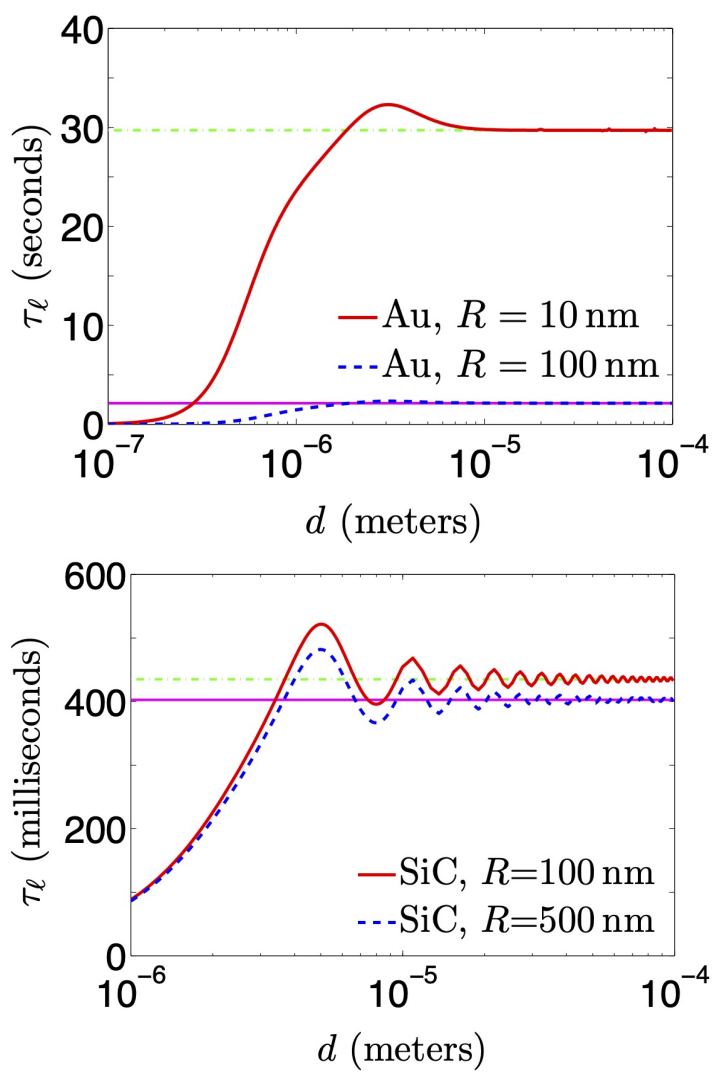

FIG. 30 Distance dependence of the relaxation time $\tau=\Gamma^{-1}$ of a nanoparticle above a substrate with temperature $T_{b}=$ $300 \mathrm{~K}$ (a) for a gold nanoparticle above a gold surface, (b) a $\mathrm{SiC}$ nanoparticle above a $\mathrm{SiC}$ surface. We use $\rho^{\mathrm{Au}} C_{\mathrm{p}}^{\mathrm{Au}}=$ $2.404 \cdot 10^{6} \mathrm{Jm}^{-3} \mathrm{~K}^{-1}$ and $\rho^{\mathrm{SiC}} C_{\mathrm{p}}^{\mathrm{SiC}}=2.212 \cdot 10^{6} \mathrm{Jm}^{-3} \mathrm{~K}^{-1}$. From (Tschikin et al. $2012 \mathrm{~b}$ ).

thermal relaxation rate ressembles the spontaneous emission rate if the nanoparticles have a narrowband emission spectrum.

In Fig. 30 it can be nicely seen that the thermal relaxation time changes by orders of magnitude when going from the far-field into the near-field regime which is due to the strong increase in $G_{1 b}$, i.e. the local density of states, in the near-field regime (Dorofeyev and Vinogradov, 2011; Joulain et al. 2003). There is also a large difference for metallic and dielectric nanoparticles due to the fact that thermal radiation is more efficient for dielectric than for metals. Furthermore, it can be seen that for SiC oscillations in the transition region between nearfield and far-field regime which can be interpreted as the photonic counterpart of the Friedel oscillations (Joulain et al. 2003). These oscillations are due to the oscillations in the local density of states which average out for the gold nanoparticle (broad band thermal emission spectrum) but remain for the $\mathrm{SiC}$ nanoparticle (narrow band thermal emission spectrum). A detailed discussion can be found in (Tschikin et al. 2012b).

\section{Dynamical control}

A control of the magnitude of heat flux has been highlighted in layered many-body systems (He et al., 2019a) coated by graphene sheets simply by tuning the doping level of graphene. Beyond this control several principles have been introduced during the last decade to dynamically control both the magnitude and the direction of heat flux at nanoscale with many-body systems. For example, by changing the shape and orientation of elements (Nikbakht, 2014) the heat flux can be modulated by several orders of magnitude with anisotropic particles as shown in Fig. 31(a). Another example for a dynamical modulation which can by realized by electrical gating is the heat flux splitter as sketched in Fig. 31(b). It enables to control the direction of the heat flux in the near-field regime. The design is based on a network of tunable graphene palets (Ben-Abdallah et al. 2015) which allow us to control spatially the near-field interactions and therewith the direction of heat flux by dynamically tuning the graphene plasmons. A similar control also has been performed with polar particles covered by graphene (Song et al. 2019).

Recently it could be demonstrated that the flux exchanged between two solids can even be amplified through a transistor effect (Ben-Abdallah and Biehs, 2014) by using a phase-change material like $\mathrm{VO}_{2}$ for an intermediate relay also called gate between two $\mathrm{SiO}_{2}$ slabs functioning as source and drain at temperatures $T_{S}=360 \mathrm{~K}$ and $T_{D}=300$ as illustrated in Fig. 32 . Since this configuration corresponds to two oppositly connected heat radiation diodes (Ben-Abdallah and Biehs, 2013; Fiorino et al., 2018a, Gu et al. , 2015: Ito et al., 2014, Yang et al. 2013) this transistor corresponds to a bipolar transistor so that the terminology emitter, base, and collector would be more appropriate but this has no impact on the physics involved. In the region of the phasetransition around its critical temperature $T_{c} \approx 340 \mathrm{~K}$ even though the temperature difference between the gate and the drain is increased a drastic reduction of flux $\Phi_{D}$ received by the drain takes place due to the strong change in the optical properties of the $\mathrm{VO}_{2}$ gate from a dielectric to a metallic response shielding the heat flux from the source towards the drain as can be seen in Fig. 32(b). This variation corresponds to the presence of a negative differential thermal conductance or resistance (Li et al. 2006) $R_{D}=\left(\frac{\partial \Phi_{D}}{\partial T_{G}}\right)^{-1}$ induced by the phase transition. In the transition region, the amplification factor

$$
a=\left|\frac{\partial \Phi_{D}}{\partial \Phi_{G}}\right|
$$

of the flux received by the drain $\Phi_{D}$ compared to the heat flux $\Phi_{G}$ removed or added to the gate can be defined. It can also be recast in terms of the thermal resistances of the source and the drain as

$$
a=\left|\frac{R_{S}}{R_{S}+R_{D}}\right|
$$



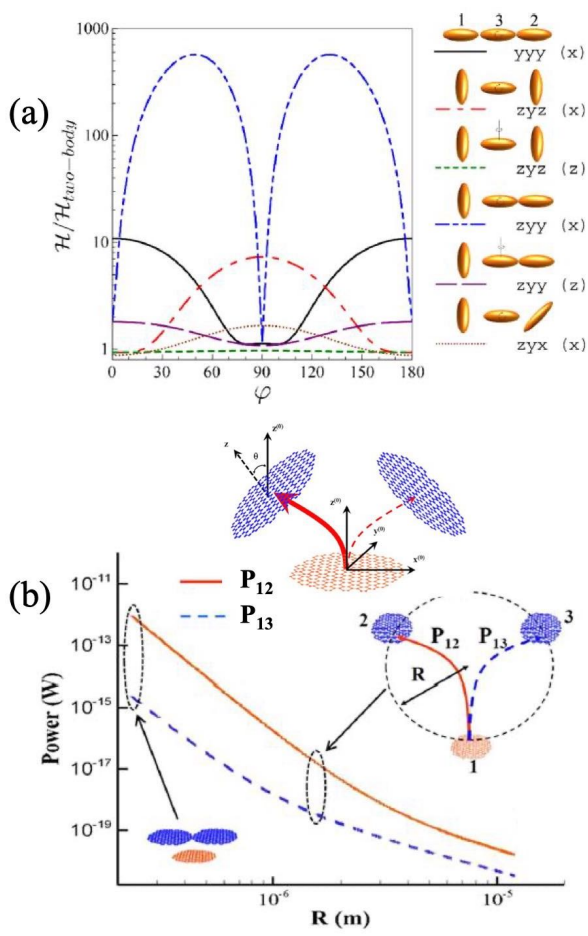

FIG. 31 (a) Normalized heat flux between two spheroidal nanoparticles with respect to the orientation of a third particle placed in between. From (Nikbakht, 2014). (b) Graphenebased heat flux splitter. Three graphene disks with different Fermi levels controlled by external gating exchange thermal energy in the near-field through many-body interactions. The magnitude of heat flow from 1 to 2 and 1 to 3 can be controlled by an appropriate tuning of the Fermi level of the graphene disks 2 and 3. The thermal power exchanged in the near-field between graphene disks of $100 \mathrm{~nm}$ radius versus the separation distance in a three body system. From (Ben-Abdallah et al. 2015).

with the postive resistance $R_{S}=-\left(\frac{\partial \Phi_{S}}{\partial T_{G}}\right)^{-1}$. This expression clearly shows that the amplification factor can only become larger than one if $R_{D}$ is negative so that a negative thermal resistance is a necessary condition for obtaining an amplification. For the thermal transistor the amplification factor is clearly larger than one in the phase-change temperature region as can be seen Fig. 32(c). Note that the peaks at the edges of the phase transition are an artefact of the effective medium model used to model the transition of the optical properties of $\mathrm{VO}_{2}$ in this region. Investigations of the same effect in the far-field regime, the impact of the hysteresis of the transistor can be found in (Joulain et al. 2015 . ProdâĂŹhomme et al. 2016, 2018) while the dynamical response of transistors can be found in (Latella et al. 2019).

The principle of negative thermal resistance plays further an important role for the so-called radiative heat shuttling which has been proposed (Latella et al. 2018b), recently. In a system consisting of only two parallel slabs, it has been shown that the periodic modulation of the (a)

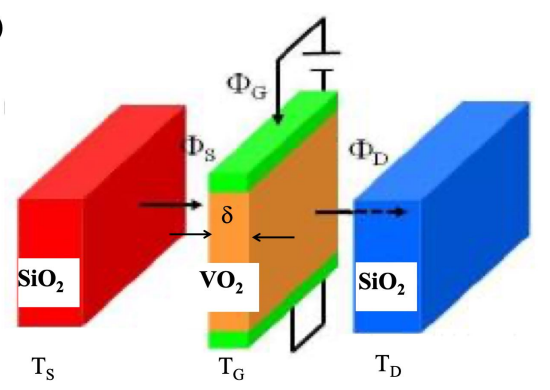

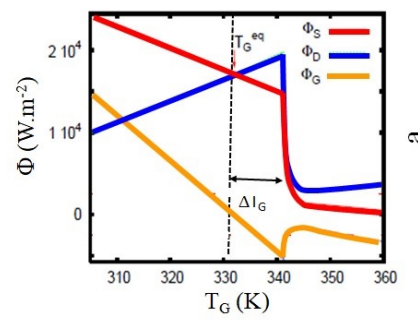

(b)

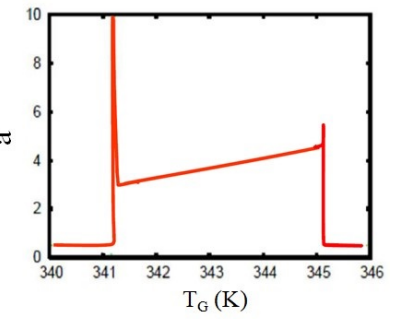

(c)
FIG. 32 (a) Radiative thermal transistor made of a threeterminal system composed of a $\mathrm{SiO}_{2}$ source, a $\mathrm{VO}_{2}$ gate and a $\mathrm{SiO}_{2}$ drain. The gate is a layer based on a phase-change material and its temperature can be actively controlled around its local equilibrium value $T_{G}^{e q}$ by an external thermostat while the temperature $T_{S}=360 \mathrm{~K}$ and $T_{D}=300$ of source and drain are fixed so that $T_{S}>T_{D}$. (b) Radiative fluxes $\Phi_{S}, \Phi_{D}$, and $\Phi_{G}$ exchanged between the different parts inside the transistor. (c) Amplification factor with respect to the gate temperature. From (Ben-Abdallah and Biehs, 2014).

temperature and/or chemical potential of the two bodies can be exploited to control the heat flux between them. More specifically, it has been proven that in order to thermally insulate them a negative thermal differential resistance is required. A further step in this direction has been done in (Messina and Ben-Abdallah, 2020), where the heat flux between two particles is tailored by periodically modulating the temperature $T_{3}$ and the position $x_{3}$ of a third particle in a three-particle system as sketched in the inset of Fig. 33. This many-body configuration allows for controlling the direction and amplitude of the heat exchanged between the two particles 1 and 2, even when they are kept at the same temperature and (differently from the shuttling effect mentioned above) in the absence of a negative thermal differential resistance (Messina and Ben-Abdallah, 2020). This possibility can be anticipated already by performing a Taylor expansion up to second order, around the middle position $x_{3}=0$ and the equi- 


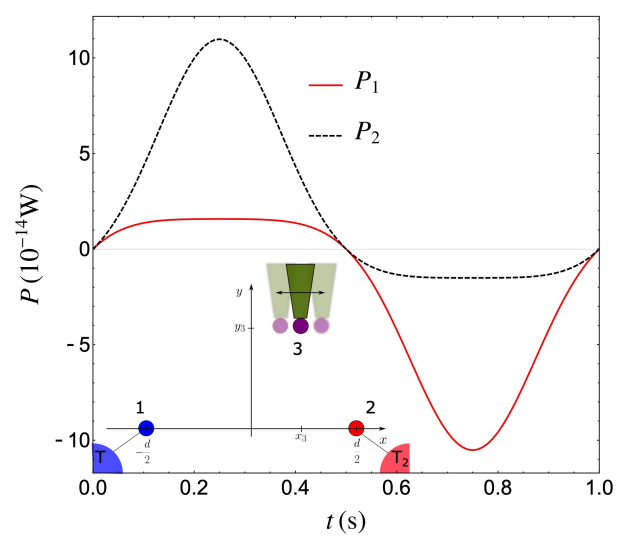

FIG. 33 Inset: geometrical configuration of a three-particle system, where the position of particle 3 is periodically modulated. Main part of the figure: radiative heat pumping by modulation of control parameters in a three-particles system sketched in the inset. The three particles are made of SiC. In this specific case, particles 1 and 2 are thermostated at temperature $T_{1}=T_{2}$ while the temperature $T_{3}$ and the $x_{3}$ coordinate of particle 3 can be modulated with respect to time. Powers $\mathcal{P}_{1}$ and $\mathcal{P}_{2}$ absorbed by particles 1 (solid red line) and 2 (dashed black line) as a function of time for the a periodic variation of the coordinate and temperature of particle 3 of frequency $\omega=2 \pi \mathrm{s}^{-1}$ and amplitudes $\Delta x=100 \mathrm{~nm}$ and $\Delta T=5 \mathrm{~K}$ around $x_{3}=0$ and $T_{3}=300 \mathrm{~K}$. We have $d=600 \mathrm{~nm}$ and $y_{3}=300 \mathrm{~nm}$, and the radius of the particle is $R=50 \mathrm{~nm}$. From (Messina and Ben-Abdallah, 2020).

librium temperature $T_{3}=T_{3 \text {,eq }}$ of particle 3 . This gives

$$
\begin{aligned}
\mathcal{P}_{1} & \simeq \mathcal{P}_{1}\left(0, T_{3, \text { eq }}\right)+\frac{\partial \mathcal{P}_{1}}{\partial x_{3}} x_{3}+\frac{\partial \mathcal{P}_{1}}{\partial T_{3}}\left(T_{3}-T_{3, \text { eq }}\right) \\
& +\frac{1}{2} \frac{\partial^{2} \mathcal{P}_{1}}{\partial x_{3}^{2}} x_{3}^{2}+\frac{1}{2} \frac{\partial^{2} \mathcal{P}_{1}}{\partial T_{3}^{2}}\left(T_{3}-T_{3, \text { eq }}\right)^{2} \\
& +\frac{\partial^{2} \mathcal{P}_{1}}{\partial x_{3} \partial T_{3}} x_{3}\left(T_{3}-T_{3, \text { eq }}\right)
\end{aligned}
$$

For a time variation of the form $T_{3}(t)=T_{3, \text { eq }}+$ $\Delta T \sin (\omega t)$ and $x_{3}(t)=\Delta x \sin (\omega t+\phi)$, and in the specific case $T_{1}=T_{2}=T_{3 \text {,eq }}$, the time average over a period reads

$$
\left\langle\mathcal{P}_{1}\right\rangle_{t} \simeq \frac{\Delta T}{2}\left(\Delta x \frac{\partial^{2} \mathcal{P}_{1}}{\partial x_{3} \partial T_{3}} \cos \phi+\frac{\Delta T}{2} \frac{\partial^{2} \mathcal{P}_{1}}{\partial T_{3}^{2}}\right),
$$

This equality clearly shows that magnitude of the first term can be easily modulated simply by changing the dephasing $\phi$ between $x_{3}$ and $T_{3}$, paving the way to an active heat pumping mechanism. More intringuing, the sign can be changed as well so that the heat can flow from cooler to warmer regions. A numerical example of this modulation for a vanishing dephasing $\phi=0$ is shown in Fig. 33, where the average over a period of the powers $P_{1}$ and $P_{2}$ absorbed by particles 1 and 2 (having temperatures $T_{1}=T_{2}=300 \mathrm{~K}$ ) are positive and negative, respectively.

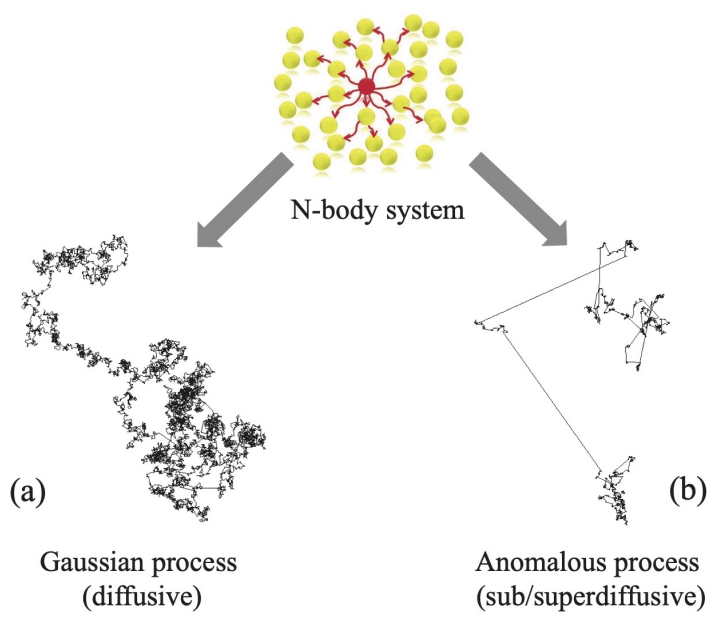

FIG. 34 Types of heat transport regimes in a $N$-body system. When an element (red) is heated up its heat spread out through the system either by (a) a classical (Gaussian) diffusion process or (b) an anomalous process. The trajectories correspond to random walks with a Gaussian and a non-Gaussian probability distribution function, respectively. Here, the non-Gaussian process is a Levy flight with an algebraic jpg.

\section{Heat transport regimes}

It is commonly admitted that heat conduction inside a bulk solid is governed by a normal diffusion process. Heat carriers that are electrons or phonons move through the atomic lattice following a usual random walk which is driven by a Gaussian distribution function as in Fig. 34(a). In this section we discuss how heat carried by thermal photons is transported in many-body systems. We demonstrate the existence of anomalous regimes of transport as in Fig. 34(b). In dilute systems we show that heat can spread out following a superdiffusive process (Lévy, 1937, Shlesinger et al. 1995) while in dense systems it can be ballistically transported.

To start this analysis, let us consider a network of small objects at temperature $T_{i}$ which are distributed inside an background or environment at temperature $T_{b}$. When the separation distance between two arbitrary objects in this network is much larger than their characteristic size and that their size is small enough compared with the thermal wavelengths $\lambda_{T_{i}}=c \hbar /\left(k_{\mathrm{B}} T_{i}\right)$ then this network can be modelled as a set of simple dipoles located at positions $\mathbf{r}_{\mathbf{i}}$ in mutual interaction and in interaction with the surrounding bath. In near-field regime the power exchanged with the bath is negligible as discussed in Sec. III.C.4 compared to the internal exchanges. Then the time evolution of objects temperature is governed by Eq. (93) neglecting the heat exchange with the background yielding

$$
I_{i} \frac{\mathrm{d} T_{i}}{\mathrm{~d} t}=\sum_{j \neq i} G_{i j}\left(T_{j}-T_{i}\right),
$$

where $I_{i}$ represents the thermal inertia of object $i$ while 

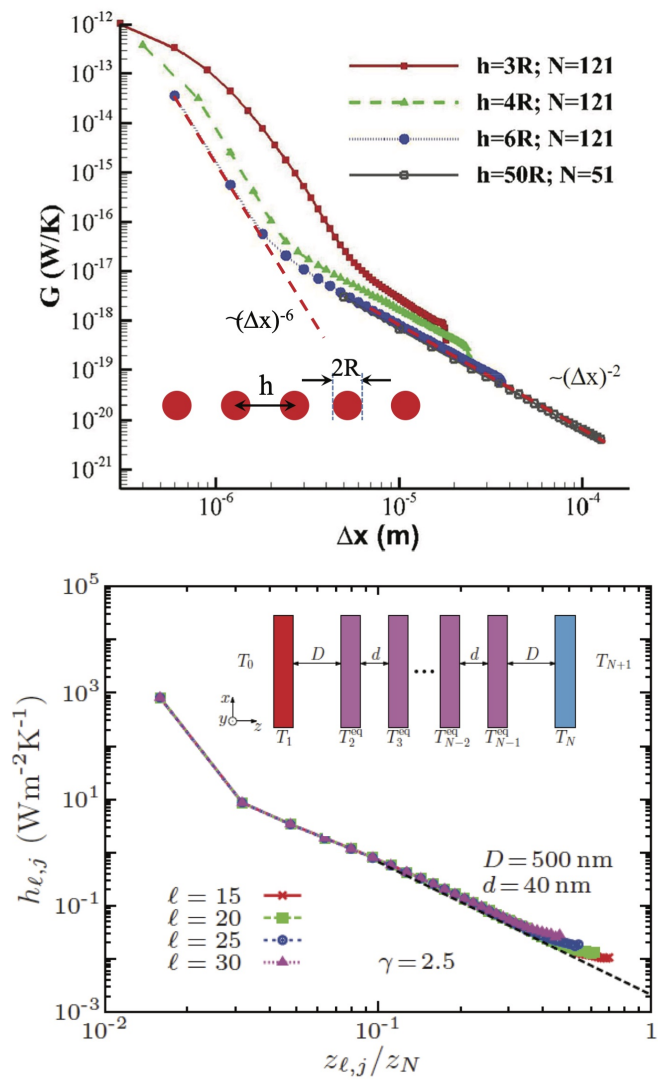

FIG. 35 (a)Thermal conductance $G$ in $\log$-log scale along a chain of SiC spherical particles $100 \mathrm{~nm}$ radius with different inter-particle distances $h$ and different particle numbers $N$ as a function of the separation distance $\Delta x=\left|\mathbf{r}-\mathbf{r}^{\prime}\right|$ at temperature $T=300 \mathrm{~K}$. From (Ben-Abdallah et al., 2013). (b) Heat-transfer coefficients $h_{\ell, j}$ with respect to the normalized separation $z_{\ell, j} / z_{N}$ in a dilute multilayer system made with $\mathrm{SiC}$ layers $200 \mathrm{~nm}$ thick separated by a distance $\mathrm{d}=40 \mathrm{~nm}$ at $T=300$ K. From (Latella et al. 2018a).

$G_{i j}$ stands for the thermal conductance between the $j^{\text {th }}$ and the $i^{t h}$ dipole as defined in Eq. (95) which depends only on the distance between the dipoles

$$
G_{i j} \equiv G\left(\left|\mathbf{r}_{i}-\mathbf{r}_{j}\right|\right) \text {. }
$$

In the continuous limit the energy balance equation (104) can be recast as (Ben-Abdallah et al. 2013)

$$
\frac{\partial T_{i}}{\partial t}=\int_{R^{d}} \mathrm{~d} \mathbf{r} p\left(\mathbf{r}_{i}, \mathbf{r}\right) \frac{T(\mathbf{r}, t)}{\tau(\mathbf{r})}-\frac{T\left(\mathbf{r}_{i}, t\right)}{\tau\left(\mathbf{r}_{\mathbf{i}}\right)},
$$

where the integration is done over the whole space of dimension $d$. This equation is formally analog to a Chapman-Kolmogorov master equation which drives a generalized Markov process. The temperature field $T(\mathbf{r}, t)$ is a passive scalar which evolves by following a generalized random walk of probability distribution function (jpg)

$$
p\left(\mathbf{r}, \mathbf{r}^{\prime}\right)=\frac{G\left(\left|\mathbf{r}-\mathbf{r}^{\prime}\right|\right)}{\int_{R^{d}} \mathrm{~d} \mathbf{r}^{\prime} G\left(\left|\mathbf{r}-\mathbf{r}^{\prime}\right|\right)}
$$

and the rate of jumps between two collission events

$$
\tau(\mathbf{r})=\left(\int_{R^{d}} \mathrm{~d} \mathbf{r}^{\prime} G\left(\left|\mathbf{r}-\mathbf{r}^{\prime}\right|\right)\right)^{-1} .
$$

Hence, by analyzing the spatial variation of the jpg and therefore of the conductance as well between two points inside the system we can identify the regime of heat transport. If the asymptotic behavior of the jpg $p(x)$ (where we have set $x=\left|\mathbf{r}-\mathbf{r}^{\prime}\right|$ ) is Gaussian, all its moments $M^{(n)}=\int x^{n} p(x) d x$ are finite so that the regime of transport is diffusive. On the other hand if it decays algebraically, i.e. $p(x)=O\left(1 / x^{\gamma}\right)$ and hence $G(x)=O\left(1 / x^{\gamma}\right)$, then there is a given order $\tilde{n}$ beyond which $M^{(n)}$ diverges for any $n>\tilde{n}$. In this case, the heat transport regime becomes superdiffusive (see right trajectory on Fig 34). In this specific case the (continuous) energy balance equation takes the form (Ben-Abdallah et al. 2013).

$$
I \frac{\partial T}{\partial t}=-\kappa(-\Delta)^{(\gamma-d) / 2} T(\mathbf{r}),
$$

where $\kappa$ is a parameter which depend on the dimension $d$ and $(-\Delta)^{\alpha / 2}$ denotes the fractional Laplacian (Shlesinger et al. 1995)

$$
(-\Delta)^{\alpha / 2} T(\mathbf{r})=c_{d ; \alpha} \mathrm{PV} \int_{R^{d}} \mathrm{~d} \mathbf{r}^{\prime} \frac{T(\mathbf{r})-T\left(\mathbf{r}^{\prime}\right)}{\left|\mathbf{r}-\mathbf{r}^{\prime}\right|^{d+\alpha}}
$$

with $c_{d ; \alpha}=\frac{2^{-\alpha} \pi^{1+d / 2}}{\Gamma(1+\alpha / 2) \Gamma\left(\frac{d+\alpha}{2}\right) \sin (\alpha \pi / 2)}$. It is worthwhile to note that Eq. 109 is general and can be applied for describing the energy balance in arbitrary dipolar or macroscopic systems. When $\gamma \rightarrow d+2$ the fractional Laplacian degenerates into its classical form, i.e. $\left.(-\Delta)^{\alpha / 2}\right)=(-\Delta)$, and the transport regime is diffusive. On the other hand when $\gamma \rightarrow d$ the fractional Laplacian approaches the identity operator and the transport becomes ballistic. Finally when $d<\gamma<d+2$ the regime is superdiffusive.

In Figs 35 and 36 we show the existence of those regimes in two simple many body systems: (1) linear chains of nanoparticles periodically dispersed in vacuum and (2) multilayer periodic systems. In the first system (see Fig. 35(a)), the thermal conductance $G(\Delta x)$ between a central particle and another particle at a distance $\Delta x$, is calculated for different filling factors $(2 R / h)$. For any filling factor, we see that $G$ decays asymptotically at long separation distance as $1 / \Delta x^{2}$, i.e. $\gamma=2$, showing according to our previous discussion that the regime of heat transport is superdiffusive. In the example plotted in Fig. 35(a) the long range interactions which give rise to this anomalous regime comes from the presence of collective electromagnetic modes supported by the whole structure. In the case of a chain made with silicon carbide ( $\mathrm{SiC}$ ) particles these modes result from the coupling of surface phonon-polaritons localized on each particle (Ben-Abdallah et al., 2013, Kathmann et al. 2018, Ordonez-Miranda et al., 2015, Tervo et al. 2020). 


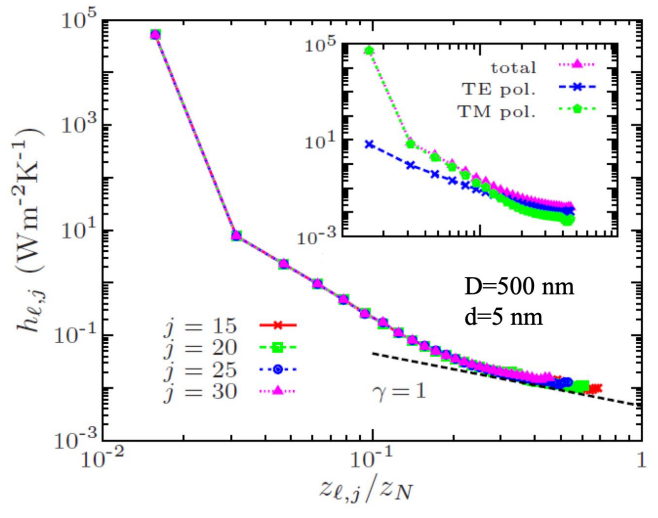

FIG. 36 Heat transfer coefficient $h_{\ell, j}$ with respect to the normalized separation $z_{l, j} / z_{N}$ in a dense multilayer system made with $\mathrm{SiC}$ layers $200 \mathrm{~nm}$ thick separated by a distance $\mathrm{d}=5$ $\mathrm{nm}$ at $T=300 \mathrm{~K}$ The inset decomposes $h_{\ell, j}$ into TE and TM polarization contributions. From (Latella et al. 2018a).

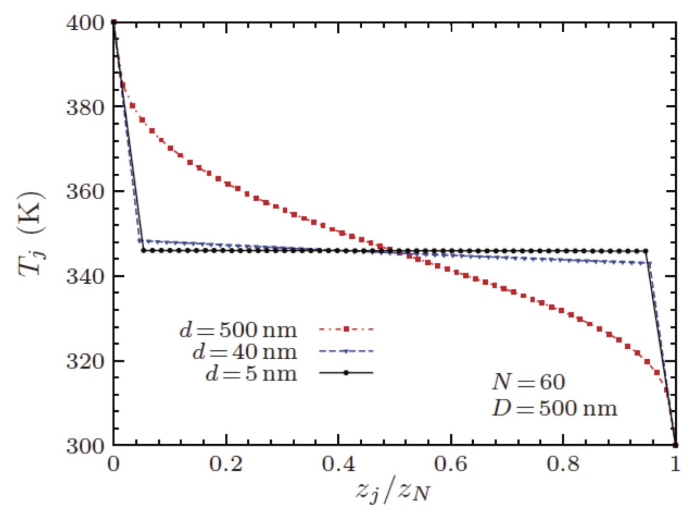

FIG. 37 Temperature profile as a function of the normalized position $z_{j} / z_{N}$ along a multilayer made with $N=60$ $\mathrm{SiC}$ layers $200 \mathrm{~nm}$ thick for different separation distances $d$ and at fixed distance $D=500 \mathrm{~nm}$ from the thermostats. From (Latella et al. 2018a).

A similar superdiffusive regime is observed in dilute multilayer systems (see Fig. 35(b)) where the heat transfer coefficient $h_{l, j}$ between layers $l$ and $j$ decays algebraically and scales as $1 / z_{l, j}^{2.5}$ where $z_{l, j}$ is the distance between layers $l$ and $j$ so that $\gamma=2.5$. On the other hand, in a dense multilayer system as considered in Fig. 35(b) a transition occurs between this superdiffusive regime and a ballistic regime (He et al., 2019c; Latella et al. 2018a). In this case we see that $\overline{h_{l, j}}$ scales as $1 / z_{l, j}$ meaning that the transport becomes clearly ballistic and the temperature profile inside the structure submitted to a temperature gradient is constant as can be seen in Fig. 37 having a value $T^{*}$ which is close to the Casimir temperature $T_{C}=\frac{T_{1}+T_{N}}{2}$. This regime of heat transport seems to be inconsistent with the previous arguments about the collective modes supported by the structure, but it occurs due to the fact that the coupling of the inner dense multilayers is much stronger than the coupling to the two outer baths when $d \ll D$. For $D=d$ on the other hand the temperature profile in Fig. 37 is reminiscent of a quasi-ballistic temperature distribution. Although the transition mechanism remains today partially elusive it has been shown in (Latella et al. 2018a) that it is related to a change of channel for heat exchanges in dense systems from TM dominated to TE dominated heat transfer (see inset of Fig. 36). For this TE polarization state the slabs do not support anymore surface waves.

\section{Non-reciprocal systems}

In electromagnetics, a nonreciprocal system is defined as a system that exhibits different received-transmitted field ratios when a source and a detector are interchanged. This concept is also closely related to a time reversal symmetry breaking of MaxwellâÁŹs equation. In this case the classical LorentzâÁŹs reciprocity is violated (Caloz et al., 2018). Here below we discuss first the general formulation of radiative power exchange between non-reciprocal objects and then show how RHT is taking place in non-reciprocal many-body systems made for sets of simple non-reciprocal nano-particles.

\section{General discussion}

As a first step, let us consider only two objects 1 and 2 having temperatures $T_{1}$ and $T_{2}$, respectively, which are immersed into a background or environment having another temperature $T_{\mathrm{b}}$. Under the assumption that the objects and the environment can be considered to be in local thermal equilibrium, the power absorbed by object 1 can be determined with the conventional FE approach analogous to Eq. (54) as (Herz and Biehs, 2019, Latella and Ben-Abdallah 2017)

$$
\mathcal{P}_{1}=3 \int_{0}^{\infty} \frac{\mathrm{d} \omega}{2 \pi} \hbar \omega\left[\left(n_{1}-n_{\mathrm{b}}\right) \mathcal{T}_{11}+\left(n_{2}-n_{\mathrm{b}}\right) \mathcal{T}_{12}\right],
$$

where $n_{1 / 2}=n\left(T_{1 / 2}\right)$ and $n_{\mathrm{b}}=n\left(T_{\mathrm{b}}\right)$. The transmission coefficients $\mathcal{T}_{\alpha / \beta}$ are, for example, explicitly given in terms of the $T$ operators of the objects in (Herz and Biehs, 2019) where they were derived within the scattering approach (Krüger et al. 2012). Here we only give explicitly the expression for $\mathcal{T}_{21}$ which is given by (Herz and Biehs, 2019)

$$
\mathcal{T}_{12}=\frac{4}{3} \operatorname{Tr}\left[\mathbb{D}^{-1} \mathbb{G} \chi_{2}\left(\mathbb{D}^{-1} \mathbb{G}\right)^{\dagger} \tilde{\chi}_{1}\right],
$$

where the trace is the operator trace, $\mathbb{G}$ is the operator for the Green's function, $\mathbb{D}=\left(\mathbb{1}-\mathbb{G T}_{2} \mathbb{G T}_{1}\right)$ written in terms of the T-operators $\mathbb{T}_{1 / 2}$ of both objects and the generalized suszeptibilities are defined as

$$
\begin{aligned}
& \chi_{2}=\frac{\mathbb{T}_{2}-\mathbb{T}_{2}^{\dagger}}{2 \mathrm{i}}-\mathbb{T}_{2} \frac{\mathbb{G}-\mathbb{G}^{\dagger}}{2 \mathrm{i}} \mathbb{T}_{2}^{\dagger}, \\
& \tilde{\chi}_{1}=\frac{\mathbb{T}_{1}-\mathbb{T}_{1}^{\dagger}}{2 \mathrm{i}}-\mathbb{T}_{1}^{\dagger} \frac{\mathbb{G}-\mathbb{G}^{\dagger}}{2 \mathrm{i}} \mathbb{T}_{1} .
\end{aligned}
$$


Note that this expression is formally equivalent to the expressions in Eqs. (64) and (74). Analogous expressions can also be found in the work (Zhu et al., 2018) and more explicitly for spherical nanoparticles in (Ott and Biehs, 2020). The corresponding expression for the absorbed power $\mathcal{P}_{2}$ in object 2 can be obtained by exchanging $1 \leftrightarrow 2$ in the above expression. First of all, it can now easily be seen in Eq. (111) that in global thermal equilibrium the overall absorbed power is zero. Secondly, when setting $T_{1}=T_{\mathrm{b}}$ then the expression in Eq. (111) can only describe the absorbed power in object 1 due to the heat flow coming from or going towards object 2 . Thus, $\mathcal{T}_{12}$ can be identified as the transmission coefficient describing the heat flow from object 2 to 1 . Thirdly, when assuming that $T_{2}=T_{\mathrm{b}}$ then Eq. (111) describes the heat flow from object 1 to the environment and to object 2 or vice versa. Therefore we can identify $\mathcal{T}_{11}$ as the transmission coefficient standing for the so called "self emission" of object 1 (Krüger et al. 2012). Finally, when taking $T_{1}=T_{2}$ then Eq. (111) describes merely the power flowing from the environment towards object 1 either directly or via object 2 . Therefore $\mathcal{T}_{11}+\mathcal{T}_{12}$ equals the transmission coefficient $\mathcal{T}_{1 \mathrm{~b}}$ as discussed also for $N$ dipolar objects when deriving Eq. (56). These observations allows us to rewrite Eq. 111) as

$$
\begin{aligned}
\mathcal{P}_{1} & =3 \int_{0}^{\infty} \frac{\mathrm{d} \omega}{2 \pi} \hbar \omega\left[n_{1} \mathcal{T}_{11}+n_{2} \mathcal{T}_{12}-n_{\mathrm{b}} \mathcal{T}_{1 \mathrm{~b}}\right] \\
& \equiv \mathcal{P}_{1 \rightarrow 1}\left(T_{1}\right)+\mathcal{P}_{2 \rightarrow 1}\left(T_{2}\right)+\mathcal{P}_{\mathrm{b} \rightarrow 1}\left(T_{\mathrm{b}}\right)
\end{aligned}
$$

introducing

$$
\begin{aligned}
& \mathcal{P}_{1 \rightarrow 1}\left(T_{1}\right)=+3 \int_{0}^{\infty} \frac{\mathrm{d} \omega}{2 \pi} \hbar \omega n_{1} \mathcal{T}_{11}, \\
& \mathcal{P}_{2 \rightarrow 1}\left(T_{2}\right)=+3 \int_{0}^{\infty} \frac{\mathrm{d} \omega}{2 \pi} \hbar \omega n_{2} \mathcal{T}_{12}, \\
& \mathcal{P}_{\mathrm{b} \rightarrow 1}\left(T_{\mathrm{b}}\right)=-3 \int_{0}^{\infty} \frac{\mathrm{d} \omega}{2 \pi} \hbar \omega n_{\mathrm{b}} \mathcal{T}_{1 \mathrm{~b}} .
\end{aligned}
$$

where the first term stands for the self-emission of body 1 , the second is the emission toward body 2 and the last term is the power coming from the bath. Notice that when the two bodies are set at the same temperature we can make a connection between the transmission coefficient $\mathcal{T}_{\mathrm{b} \rightarrow 1}$ and the thermal emissivity $\epsilon=\sigma_{\text {abs }} / S$ (Biehs and Ben-Abdallah, 2016). More specifically, $\mathcal{T}_{\mathrm{b} \rightarrow 1}$ can be expressed as a function of its absorption-cross section (30), its geometrical cross section $S$ and the absorbed power as follows

$$
\mathcal{T}_{\mathrm{b} \rightarrow 1}(\omega)=\frac{A}{2 \pi} \epsilon(\omega) \frac{\omega^{2}}{c^{2}}=\frac{A}{2 \pi} \frac{\sigma_{\mathrm{abs}}(\omega)}{S} \frac{\omega^{2}}{c^{2}},
$$

where $A$ is the surface of the object (assumed convex).

The self-emission term $\mathcal{P}_{1 \rightarrow 1}$ appearing in Eq. (116) must balance the energy flow from the other object 2 and the environment described by $\mathcal{P}_{2 \rightarrow 1}$ and $\mathcal{P}_{\mathrm{b} \rightarrow 1}$ to establish global equilibrium so that this term describes the power needed to keep the temperature of object 1 constant in thermal equilibrium. Hence, when taking $T_{1}=T_{2}=T_{\mathrm{b}}$ we have $\mathcal{P}_{1}=0$ and therefore

$$
\mathcal{P}_{1 \rightarrow 1}\left(T_{\mathrm{b}}\right)=-\mathcal{P}_{2 \rightarrow 1}\left(T_{\mathrm{b}}\right)-\mathcal{P}_{\mathrm{b} \rightarrow 1}\left(T_{\mathrm{b}}\right)
$$

This equation relates $\mathcal{P}_{1 \rightarrow 1}$ to $\mathcal{P}_{2 \rightarrow 1}$ and $\mathcal{P}_{\mathrm{b} \rightarrow 1}$ and therefore allows us to eliminate the background term $\mathcal{P}_{\mathrm{b} \rightarrow 1}\left(T_{\mathrm{b}}\right)$ from the expression for the overall absorbed power giving (Krüger et al. 2012)

$$
\begin{aligned}
\mathcal{P}_{1}= & \mathcal{P}_{1 \rightarrow 1}\left(T_{1}\right)-\mathcal{P}_{1 \rightarrow 1}\left(T_{\mathrm{b}}\right) \\
& +\mathcal{P}_{2 \rightarrow 1}\left(T_{2}\right)-\mathcal{P}_{2 \rightarrow 1}\left(T_{\mathrm{b}}\right)
\end{aligned}
$$

This elimination of the background term is of course clear from the above definitions showing that $\mathcal{T}_{1 \rightarrow \mathrm{b}}$ can also be expressed by $\mathcal{T}_{1 \rightarrow 1}$ and $\mathcal{T}_{2 \rightarrow 1}$ and obviously the implementation of the equilibrium condition brought us back to Eq. (111). As described in (Krüger et al., 2012) this expression for $\mathcal{P}_{1}$ can now be generalized to the case of $N$ objects in a given environment. In this case $(i=1, \ldots, N)$

$$
\mathcal{P}_{i}=\sum_{j=1}^{N}\left[\mathcal{P}_{j \rightarrow i}\left(T_{j}\right)-\mathcal{P}_{j \rightarrow i}\left(T_{\mathrm{b}}\right)\right] .
$$

This is the general $N$-body formula for the power absorbed by object $i$ of which Eq. (54) can be considered as a special case for dipolar objects. For an explicit calculation of the absorbed power it is, of course, necessary to determine the transmission coefficients for the studied configuration. Before focusing on the heat flow in some specific cases, we want to discuss in the next section the impact of the non-reciprocity in a similarly general way.

\section{General impact of non-reciprocity}

For Lorentz-reciprocal objects and their environment the coresponding response functions, i.e. the permittivity tensor, the polarizability tensor, T-operator, Green's function etc. are symmetric (Caloz et al. 2018). Consequently, in this case we have symmetric transmission coefficients $\mathcal{T}_{12}=\mathcal{T}_{21}$ or more generally for $N$ objects $\mathcal{T}_{i j}=\mathcal{T}_{j i}(i \neq j)$. This means that we have detailed balance for the heat flux between any two objects (Krüger et al. 2012). In contrast, for configurations where the objects or the environment do not fulfill the conditions for Lorentz reciprocity it has been explicitly proven in (Herz and Biehs, 2019) that in general $\mathcal{T}_{12} \neq \mathcal{T}_{21}$. More precisely, $\mathcal{T}_{12}=\mathcal{T}_{21}$ if and only if the objects and their environment are both reciprocal. Therefore non-reciprocity introduces in general a directionality for the heat flow.

One of the astonishing consequences is that in nonreciprocal systems one has $\mathcal{P}_{12} \neq \mathcal{P}_{21}$ in general so that the heat flux related expressions for the reciprocal case fulfilling detailed balance need to be generalized to the non-reciprocal case where detailed balance is broken (Zhu and Fan, 2014) like, for instance, the Green-Kubo relation for heat radiation (Golyk et al. 2013, Herz and 
Biehs, 2019). More surprisingly, this asymmetry in the heat flow from object 1 to object 2 and from 2 to 1 even exists in global thermal equilibrium suggesting that there might be a net heat flow even though there is no temperature difference. However, by looking at Eq. (111) it is clear that although the heat flux from object 1 towards object 2 is different from the heat flux from object 2 to 1 there is no net heat flow in global equilibrium because $\mathcal{P}_{1}=\mathcal{P}_{2}=0$ in that case. The same is also true for $N$ objects where due to non-reciprocity one has in general $\mathcal{P}_{i \rightarrow j} \neq \mathcal{P}_{j \rightarrow i}(i \neq j)$. As we discuss below in more detail, this can result in a so-called persistent heat current in a $N$-body configuration in global thermal equilibrium (Ott et al., 2019a; Zhu and Fan, 2016, Zhu et al., 2018).

In many works on the radiative heat exchange between two objects the contribution of the environmental field is neglected. In that case, as pointed out in (Latella and Ben-Abdallah, 2017), the global equilibrium can only be achieved if the transmission coefficients fulfill the condition

$$
\sum_{j \neq i}\left[\mathcal{T}_{i j}-\mathcal{T}_{j i}\right]=0
$$

In particular, this implies that when having only two objects $\mathcal{T}_{12}=\mathcal{T}_{21}$. Hence, for two isolated objects the nonreciprocity has no impact and therefore at least three objects are necessary to observe for example a broken detailed balance. From this very general findings it can be understood that in (Zhu and Fan, 2014) it was necessary to consider three non-reciprocal thermal emitters to show that detailed balance can be broken for thermal radiation and in (Zhu and Fan, 2016) it was necessary to consider three non-reciprocal nanoparticles to observe the persistent heat current. On the other hand, it is also clear that the heat exchange between two non-reciprocal halfspaces will not show any rectification effect (Fan et al. 2020, Moncada-Villa et al., 2015). On the other hand, when considering two objects with an environment, then the environment can be regarded as a third object. This explains why in general for only two objects in a given environment the transmission coefficients can be asymetric $\left(\mathcal{T}_{1 \rightarrow 2} \neq \mathcal{T}_{2 \rightarrow 1}\right)$ so that we have here no contradiction to the discussion at the beginning of this paragraph.

\section{Magneto-optical nano-particles}

In the following we will review the results obtained for the RHT in many-body systems consisting of subwavelength nano-particles. Most of the works are neglecting the coupling to the background which can be justified in steady-state situations when the distance between the objects is much smaller than the thermal wavelength so that the near-field coupling dominates over the coupling to the environment (Messina et al. $2013 \mathrm{~b})$. Therefore, we will work with expression (58) together with the transmission coefficients $\mathcal{T}_{i j}$ as defined in Eq. (55). Neglecting the radiation correction it can also be written as

$$
\mathcal{T}_{i j}(\omega)=\frac{4}{3} \operatorname{Tr}\left[\underline{\underline{\alpha}}^{-1} \boldsymbol{T}_{i j}^{-1} \frac{\underline{\underline{\underline{\alpha}}}-\underline{\underline{\alpha}}^{\dagger}}{2 \mathrm{i}}\left(\underline{\underline{\alpha}}^{-1} \boldsymbol{T}_{i j}^{-1}\right)^{\dagger} \frac{\underline{\underline{\alpha}}-\underline{\underline{\alpha}}^{\dagger}}{2 \mathrm{i}}\right] .
$$

assuming that all particles have the same polarizability $\underline{\underline{\alpha}}$ defined for spherical nano-particles by means of the permittivity in Eq. (68) with the volume $V=$ $4 \pi R^{3} / 3$ (Lakhtakia et al., 1991)

$$
\underline{\underline{\alpha}}=4 \pi R^{3}(\underline{\underline{\epsilon}}-\mathbb{1})(\underline{\underline{\epsilon}}+2 \mathbb{1})^{-1} .
$$

The transmission coefficients in Eq. (124) are equal to the expressions given in (Ben-Abdallah et al., 2011; Ekeroth et al. 2017) for spherical nano-particles within the so called weak-coupled dipole limit (Lakhtakia, 1992) where the radiation correction can be neglected (Albaladejo et al. 2010). They can also be derived from the general $T$-operator expressions obtained within the scattering approach for the reciprocal (Krüger et al. 2012) and for the non-reciprocal case (Herz and Biehs, 2019, Zhu et al. 2018).

As already done in Sec. II.C we consider again InSb as magneto-optical material for which the permittivity tensor becomes asymmetric $\underline{\underline{\epsilon}}^{t} \neq \underline{\underline{\epsilon}}$, i.e. the material properties are non-reciprocal, when a magnetic field is applied. As a consequence, the polarizability tensor then has the same asymmetry $\underline{\underline{\alpha}} \neq \underline{\underline{\alpha}}^{t}$. Furthermore, due to the applied field the three-fold degeneracy of the dipolar localized plasmon resonances, solution of the transcendental equation $\operatorname{det}(\epsilon+2 \mathbb{1})=0$, with magnetic quantum number $m=0, \pm \overline{1}$ is lifted (Pineider et al. 2013; Weick and Weinmann, 2011). In particular, there is a red-shift of the resonance with $m=+1$ and a blue-shift of the resonance with $m=-1$. The size of the splitting is proportional to the cyclotron frequency $\omega_{c}=e B / m^{*}, m^{*}$ being the effective mass of electrons (Pineider et al., 2013, Weick and Weinmann, 2011). To be more precise, in the regime where the dissipation can be neglected we find the resonances (Ott et al. 2018)

$$
\begin{aligned}
\omega_{m=\mp 1} & =\sqrt{\left(\frac{\epsilon_{\infty} \omega_{\mathrm{p}}^{2}}{\epsilon_{\infty}+2}+\frac{\omega_{\mathrm{c}}^{2}}{4}\right)} \pm \frac{\omega_{\mathrm{c}}}{2}, \\
\omega_{m=0} & =\sqrt{\frac{\epsilon_{\infty} \omega_{\mathrm{p}}^{2}}{\epsilon_{\infty}+2}},
\end{aligned}
$$

which are deterimined by the poles of the polarizability tensor. Therefore, for small magnetic fields the two circular resonances with $m= \pm 1$ are shifted by $\mp \omega_{c} / 2$ with respect to the unaffected resonance for $m=0$.

\section{Giant magneto-resistance}

Due to the strong dependence of dipolar resonances of particles on the magnetic field the heat flux emitted by a magneto-optical particle can drastically change by tuning 


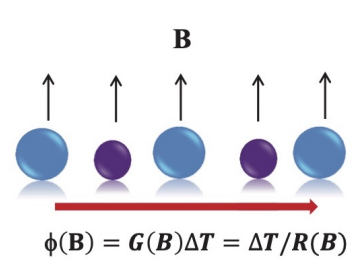

(a)

(b)

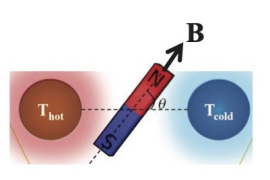

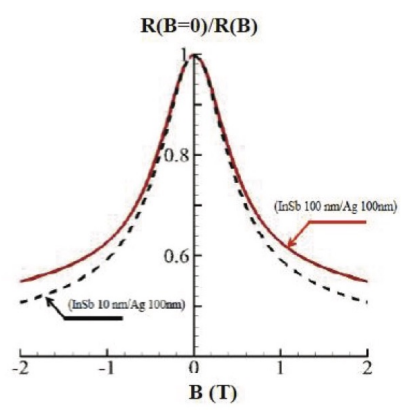

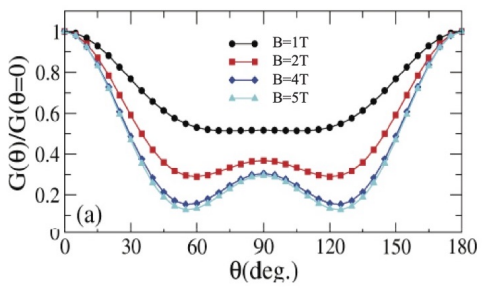

FIG. 38 (a) Giant thermal magneto-resistance along linear chains of $\mathrm{InSb}$ and $\mathrm{InSb} / \mathrm{Ag}$ nanoparticles at $T=300 \mathrm{~K}$ as a function of the strength of an external magnetic field $B$ applied in the direction orthogonal to the chain axis. From (Latella and Ben-Abdallah, 2017). (b) Anisotropic magneto-resitance between two InSb nanoparticles with respect to the orientation of magnetic field. From (Ekeroth et al. 2018).

this field (Ekeroth et al. 2018, Latella and Ben-Abdallah, 2017). It turns out that the thermal magneto-resistance

$$
R_{i j}(\mathbf{B})=\left(3 \int_{0}^{\infty} \frac{\mathrm{d} \omega}{2 \pi} \hbar \omega \frac{\partial n}{\partial T} \mathcal{T}_{i j}(\omega, \mathbf{B})\right)^{-1}
$$

between two particles in a many-body system is strongly dependent on the magnitude of applied magnetic field as it can be seen in Fig. 38(a). Variations of about $50 \%$ along nanoparticle chains has been highlighted with magnetic fields of magnitude of about $500 \mathrm{mT}$ (Latella and Ben-Abdallah, 2017). This sensitivity to the magnetic field is of the same order of magnitude than the giant electric magneto-resistance reported in ferromagnetic/normal metal multilayers (Baibich et al., 1988). This resistance can also be tuned by changing the direction of applied magnetic field (Ekeroth et al., 2018$)$. In this case we speak of an anisotropic magneto-resistance. As shown in Fig. 38(b), for certain orientations of the magnetic field the heat flux can drop by more than $90 \%$. These effects open up the opportunity to control or modulate the amplitude of the heat flux between nanoparticles by external means. A more detailed discussion can be found in (Ekeroth et al., 2018, Latella and BenAbdallah, 2017; Ott et al., 2019a).

5. Persistent heat flux, angular momentum, spin and heat current

As shown in (Ott et al. 2018) the circular plasmonic resonances for $m= \pm 1$ of a single particle responsible for magnetic circular dichroism (Pineider et al., 2013) and "inverse Faraday effect" (Gu and Kornev, 2010) are connected with a circular mean heat flux

$$
\langle\mathbf{S}\rangle=\langle\mathbf{E} \times \mathbf{H}\rangle
$$

emitted by the nano-particle in planes perpendicular to the applied magnetic field. This results in a certain spectral angular momentum density $\left\langle\mathbf{J}_{\omega}\right\rangle=\langle\mathbf{L}\rangle_{\omega}+\left\langle\mathbf{S}_{d}\right\rangle_{\omega}$ which can be divided in an orbital $\langle\mathbf{L}\rangle_{\omega}$ and spin angular momentum density $\left\langle\mathbf{S}_{\mathrm{d}}\right\rangle_{\omega}$ defined as (Bliokh and Nori 2015)

$$
\begin{aligned}
\langle\mathbf{L}\rangle_{\omega} & =\mathbf{r} \times\langle\mathbf{P}\rangle_{\omega}, \\
\left\langle\mathbf{S}_{d}\right\rangle_{\omega} & =\frac{g}{2} \operatorname{Im}\left(\left\langle\mathbf{E}^{*} \times \mathbf{E}\right\rangle+\frac{\mu_{0}}{\epsilon_{0}}\left\langle\mathbf{H}^{*} \times \mathbf{H}\right\rangle\right),
\end{aligned}
$$

with $g=\epsilon_{0} / \omega$ and the canonical spectral momentum density is given by

$$
\langle\mathbf{P}\rangle_{\omega}=\frac{g}{2} \operatorname{Im}\left[\left\langle\mathbf{E}^{*}(\nabla) \mathbf{E}\right\rangle+\frac{\mu_{0}}{\epsilon_{0}}\left\langle\mathbf{H}^{*}(\nabla) \mathbf{H}\right\rangle\right],
$$

adopting the notations from (Bliokh and Nori 2015) that $\vec{X}(\vec{Y}) \vec{Z}=\sum_{i} X_{i} \vec{Y} Z_{i}$. Using these definitions together with $\mathrm{FE}$ the persistent angular momentum close to the walls of a cavity was first evaluated and discussed in (Silveirinha, 2017) and the angular momentum and spin for a thermally emitting nanoparticle by (Ott et al., 2018). A more detailed study of the angular momentum and spin close to a planar interface has been published recently (Khandekar and Jacob, 2019a).

That there is a finite angular momentum and spin of the thermally emitted radiation is not surprising, because the Lorentz force constrains the electrons in the nanoparticles on a circular orbit so that the dipolar resonance is rotating in the plane perpendicular to the magnetic field which is the microscopic origin of the circular heat flux and the total angular momentum. The righthand rule determines the direction of the circular heat flux in the near-field regime (Ott et al., 2018). It is interesting to note that the angular momentum of the $m=+1(m=-1)$ resonance is oriented in the (opposite) direction of the magnetic field as one would expect, whereas the spin of the $m=-1(m=+1)$ is oriented in the (opposite) direction of the magnetic field in the near-field regime. From this perspective the splitting of the $m= \pm 1$ resonances can also be understood as a Zeeman splitting, where $m=-1(m=+1)$ is blueshifted (red-shifted) because the near-field direction of the spin is in direction (opposite) to the magnetic field, but of course the correct quantity determining the Zeeman splitting is the magnetic momentum of the dipolar resonance itself ( $\mathrm{Gu}$ and Kornev, 2010). The presence of a finite spin means that the thermal emission of the non-reciprocal nanoparticle will be circularly polarized in general as is well known for solid matter within a magnetic field like semi-conductors (Kollyukh et al. 2005) but also white dwarfs (Kemp, 1970 Kemp et al. 1970), 


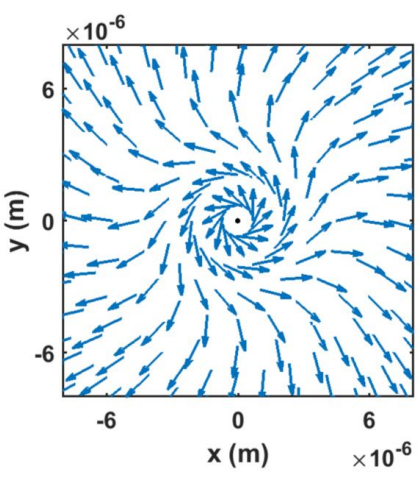

FIG. 39 Normalized mean Poynting vector $\langle\mathbf{S}\rangle$ of thermal radiation emitted by an $\mathrm{InSb}$ nanoparticle at the origin of the coordinate system with radius of $300 \mathrm{~nm}$ at a temperature of $300 \mathrm{~K}$ into a cold environment (vacuum) at $T_{b}=0 \mathrm{~K}$ when a magnetic field is applied in $z$ direction. This circular heat flux persists in global thermal equilibrium. From Ott et al. 2018)

for instance. More recently, circularly polarized thermal emitters based on chiral meta-surfaces (Dyakov et al. 2018) and nano-antennas (Khandekar and Jacob, 2019b) have been proposed.

Interestingly, it turns out that these three quantities, mean heat flux, orbital angular momentum, and spin persist in global equilibrium if $\underline{\underline{\alpha}} \neq \underline{\underline{\alpha}}^{t}$ and therefore is a direct consequence of the non-reciprocity of the permittivity or polarizability. Even though it might seem strange to have a non-zero mean heat flux in global equilibrium circulating around the nanoparticle, this does not pose any problem from the thermodynamical point of view, since it can be shown that $\nabla \cdot\left\langle\mathbf{S}_{\text {pers }}\right\rangle=0$, which means that there is no heat flux through any closed surface including the nanoparticle (Ott et al., 2018). In other words, no heat is finally emitted. Similar conclusions have been made for the thermal radiation field of the nonreciprocal surface modes on planar interfaces (Khandekar and Jacob, 2019a; Silveirinha, 2017).

Instead of a persistent heat flux, i.e. a non-zero heat flux in global thermal equilibrium, as observed from the mean Poynting vector around a non-reciprocal nanoparticle or in the vicinity of a planar interface of a nonreciprocal sample, there can also be a persistent heat current as first discussed in (Zhu and Fan, 2016) for the thermal radiation exchanged by three nanoparticles, but it of course exists also for more than three particles (Zhu et al. 2018). As clear from the above discussion, when neglecting the contribution of the environment of the nanoparticles, then it follows from the constraint in Eq. (123) that for only two nanoparticles $\mathcal{T}_{12}=\mathcal{T}_{21}$ and consequently $\mathcal{P}_{1 \rightarrow 2}=\mathcal{P}_{2 \rightarrow 1}$ if $T_{1}=T_{2}$. Therefore it is necessary to have at least three nanoparticles to have $\mathcal{T}_{12} \neq \mathcal{T}_{21}$. For three particles as in Fig. 40 the constraint in Eq. (123) demands $\mathcal{T}_{12}=\mathcal{T}_{23}=\mathcal{T}_{31}$ and $\mathcal{T}_{13}=\mathcal{T}_{32}=\mathcal{T}_{21}$ due to the $\mathrm{C}_{3}$ symmetry. If the three nanoparticles are now nonreciprocal then it can be shown from the definition of the

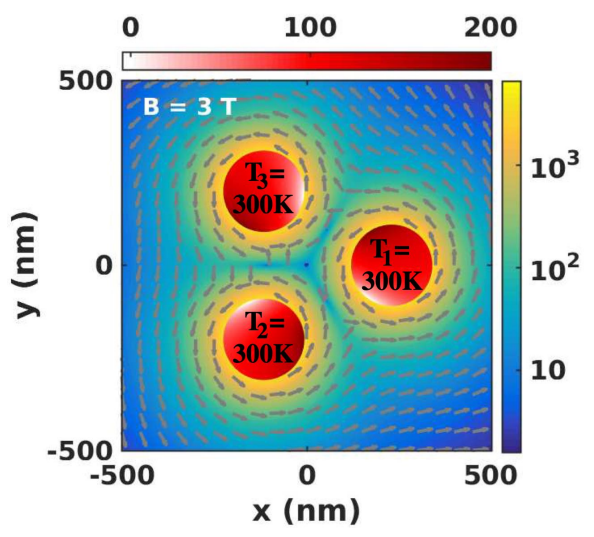

FIG. 40 Normalized mean Poynting vector $\langle\mathbf{S}\rangle$ and its magnitude $\left(\mathrm{Wm}^{-2}\right.$ in color scale) of thermal radiation emitted by three $\mathrm{InSb}$ nanoparticles with a radius of $300 \mathrm{~nm}$ having the same temperatures $T_{1}=T_{2}=T_{3}=300 \mathrm{~K}$ when a magnetic field is applied in $z$ direction. From (Ott et al. 2019b).

transmission coefficient in Eq. 124 that $\mathcal{T}_{12} \neq \mathcal{T}_{21}$ and hence

$$
\mathcal{P}_{1 \rightarrow 2}=\mathcal{P}_{2 \rightarrow 3}=\mathcal{P}_{3 \rightarrow 1} \neq \mathcal{P}_{1 \rightarrow 3}=\mathcal{P}_{3 \rightarrow 2}=\mathcal{P}_{2 \rightarrow 1} .
$$

This means there is a clockwise heat flow exchanged by the nanoparticles which is different from the heat flow in counter-clockwise direction even if $T_{1}=T_{2}=T_{3}$ and therefore there is a persistent heat current in clockwise or counterclockwise direction depending on which of the two heat flows is larger. This persistent heat flow or better heat current (Zhu and Fan, 2016) is the many-body analogue of the persistent heat flux, which of course also exists in the three-body configuration. It is worthwhile to note from relation $(123)$ that, in a non-reciprocal system at temperature $T$, the body $i$ and $j$ still exchange a power (Latella and Ben-Abdallah, 2017)

$$
\begin{aligned}
\mathcal{P}_{i \leftrightarrow j}^{e q} & =\mathcal{P}_{j \rightarrow i}^{e q}-\mathcal{P}_{i \rightarrow j}^{e q} \\
& =\int_{0}^{\infty} \frac{\mathrm{d} \omega}{2 \pi} \hbar \omega n(\omega, T)\left[\mathcal{T}_{i j}-\mathcal{T}_{j i}\right],
\end{aligned}
$$

although the net power $\mathcal{P}_{j}^{e q}=\sum_{i \neq j} \mathcal{P}_{i \leftrightarrow j}^{e q}$ vanishes so that the persistent heat flux does not lead to any heating or cooling. Hence the magnitude of asymmetry of transmission coefficients spectra (Fig. 41) and the value of the equilibrium temperature are directly responsible for the value of persistent current. Today, the measurement of this current is still a challenging problem. Recently, a setup has been proposed in Ref. (Khandekar and Jacob, 2019a) which might be able to access it in the vicinity of a magneto-optical planar sample.

\section{Hall effect for thermal radiation}

The asymmetry in the exchanged heat flux in manybody configurations observed in global equilibrium, i.e. 

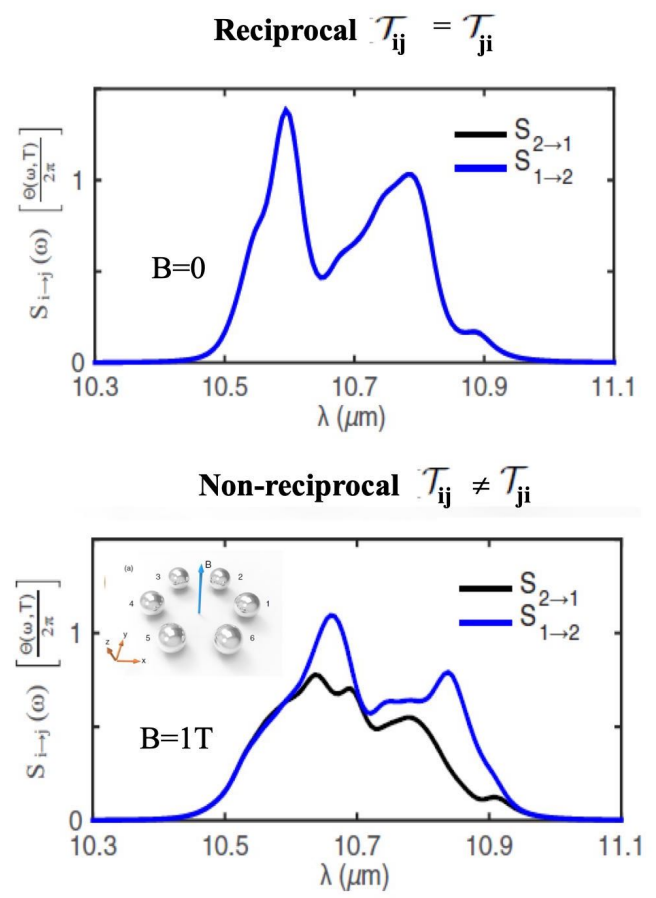

FIG. 41 Heat transfer spectra in a many-body system consisting of six InSb nanospheres placed at the vertices of a regular hexagon on $x-y$ plane without (reciprocal) and with (non-reciprocal) an externally applied magnetic field in the $z$-direction. From (Zhu and Fan, 2016 Zhu et al., 2018)

the persistent heat current, has directly measurable consequences when driving the system out of global equilibrium. An astonishing consequence is the Righi-Leduc or Hall-effect for thermal radiation (Ben-Abdallah, 2016). Classically, the Righi-Leduc effect (Leduc, 1887; Righi, 1887 ) is just the thermal analogue of the Hall-effect (Hall, 1879). When applying a temperature difference in a metallic sample together with a magnetic field the heat current by the electrons will be deflected due to the Lorentz-force acting on the electrons such that a temperature difference perpendicular to the initially applied temperature difference will build up in steady state. Such an effect has also been highlighted for other heat carriers in solids like magnons and spinons (Fujimoto, 2009, Katsura et al. 2010 Onose et al., 2010) or even phonons (Inyushkin and Taldenkov, 2007, Strohm et al. 2005).

Now, when considering heat radiation exchanged between four nanoparticles in a $\mathrm{C}_{4}$ symmetric configuration as in Fig. 42, and applying a temperature difference $\Delta T=T_{L}-T_{R}$ between particle $L$ (left) and $R$ (right), then in the steady state of the system a temperature difference $T_{B}^{(s t)}-T_{T}^{(s t)}$ between particle $B$ (bottom) and $U$ (up) can build up when using non-reciprocal InSb nanoparticles and applying a magnetic field perpendicular to the particle plane. Hence, one observes a Righi-Leduc or Hall effect for thermal radiation (Ben-Abdallah, 2016). Again, the effect can be understood by the Lorentz force

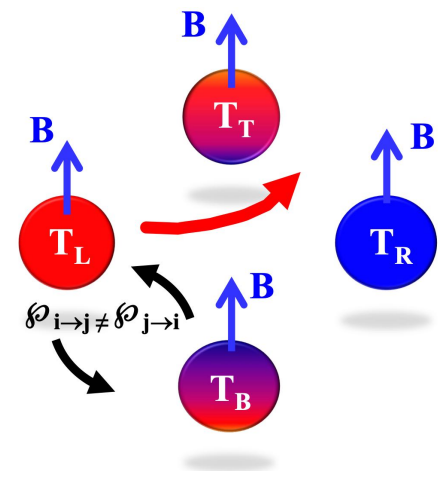

FIG. 42 Photon thermal Hall effect: a four terminal junction with magneto-optical particles forming a square with $\mathrm{C}_{4}$ symmetry is submitted to an external magnetic field $\mathbf{B}$ in the direction orthogonal particle plane. When a temperature gradient $\Delta T=T_{L}-T_{R}$ is applied between the particles $L$ and $R$, a Hall flux transfers heat transversally between particles $B$ and $T$, thus bending the overall flux (red arrow) towards the top or the bottom. In this case the heat $\mathcal{P}_{i \rightarrow j}$ and $\mathcal{P}_{j \rightarrow i}$ exchanged between two particles $i$ and $j$ is not symmetric.

acting on the electrons in the nanoparticles. However, here the electrons do not serve as the heat carriers but introduce a circular heat flux leading to an asymmetric heat flow and finally to the Righi-Leduc effect. Its magnitude and directionality can be measured by the relative Hall temperature difference or Righi-Leduc-like coefficient

$$
R_{T}=\frac{T_{B}^{(s t)}-T_{T}^{(s t)}}{T_{L}-T_{R}},
$$

which is shown in Fig. 43. Written in terms of thermal conductances, this coefficient reads (Ben-Abdallah, 2016)

$$
R_{T}=\frac{G_{31} G_{42}-G_{41} G_{32}}{\sum_{j \neq 3} G_{3 j} \sum_{j \neq 4} G_{4 j}-G_{34} G_{43}} .
$$

It can be seen that depending on the magnitude of the magnetic field the effect will change its directionality and there is a maximum for a magnetic field amplitude of about $0.5 \mathrm{~T}$ for the considered configuration. The effect is not very strong and high field amplitudes are needed to have a maximum effect. However it highly depends on the configuration and material parameters (Ott et al. 2019b) and therefore its magnitude can certainly be optimized by changing the spatial distribution of particles or their physical properties. To date an experimental proof of photon thermal Hall effect remains a challenging problem. However a direct measurement of the Hall temperature difference with measurements of electrical resistance variations with a very high accuracy (St-Gelais et al. 2014) in magneto-optical nanowires networks seems feasible.

Beside the "normal" thermal Hall effect, anomalous effects also called anomalous thermal Hall effects (Ferreiros et al. 2017: Huang et al., 2020) thermal analog of anomalous Hall effect (Karplus and Luttinger, 1954, Nagaosa 


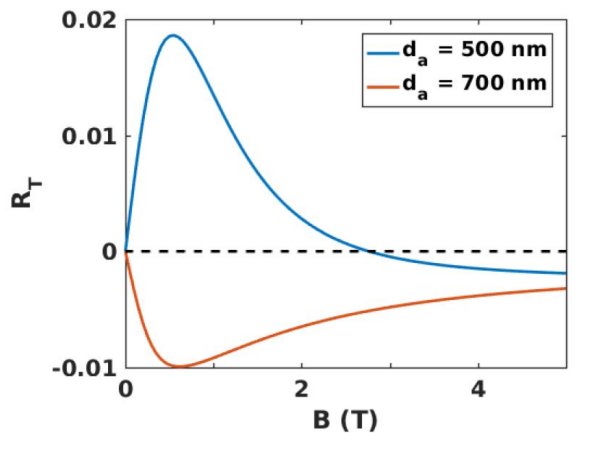

FIG. 43 Magnetic field strength dependence of the RighiLeduc-like coefficient defined in Eq. 134 for four spherical InSb nanoparticles with a radius of $100 \mathrm{~nm}$ in a $\mathrm{C}_{4}$ symmetric configuration as depicted in Fig. 42 choosing an interparticle distance of opposite particles of $d_{a}=500 \mathrm{~nm}$ and $d_{a}=700 \mathrm{~nm}$. From (Ott et al. 2019b).

et al. 2010) have also been described for the heat transport with electron or phonons in ferromagnetic materials and in semimetals. Very recently a similar effect in Weyl semi-metal nanoparticles networks for thermal photons has been predicted (Ott et al., 2020). Since the Weyl semi-metals can exhibit a strong nonreciprocal response in the infrared, this effect allows for a directional control of heat flux by simply locally tuning the magnitude of temperature field without changing the direction of temperature gradient.

\section{Heat flux rectification with non-reciprocal surface waves}

For most of the non-reciprocal effects discussed so far the environment does not play a decisive role. Now, instead of using only the intrinsical non-reciprocal properties of the nanoparticles to achieve a directional heat flux, also the non-reciprocity of the environment can be exploited as first shown in (Ott et al. 2019a). As we have seen in Sec. III.C.3 before, the heat flux between two nanoparticles or more generally between two objects brought in close vicinity to an interface of a sample can be enhanced by transporting the heat via the surface modes of the interface (Asheichyk et al. 2017; Dong et al., 2018, He et al. 2019b; Messina et al. 2018, Sääskilahti et al. 2014 Zhang et al. 2019a). If the material properties of the planar sample are non-reciprocal then the presence of a magnetic field will affect the surface modes Chiu and Quinn, 1972).

To be more specific, within the Voigt configuration as in Fig. 44(a) and (b) the dispersion relation for the surface modes at the interface of the substrate traveling to the right and left will be different (Chiu and Quinn, 1972). Similar to the localized mode inside an InSb nanoparticle the degeneracy of the surface modes for $k_{x}>0$ and $k_{x}<0$ is lifted and there is a splitting of the surface mode resonance frequency (Chiu and Quinn, 1972). Since the spin associated with the surface (a)
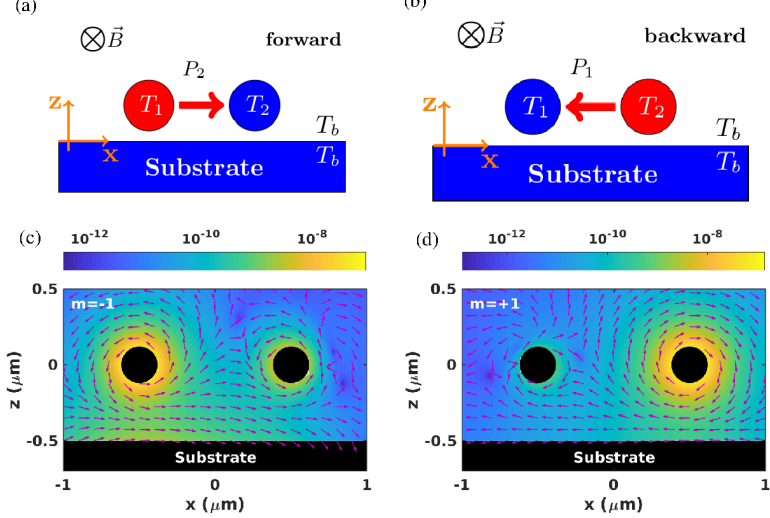

FIG. 44 (a) Sketch of the diode in forward direction. Two InSb nanoparticles above an InSb substrate. The left particle is heated with respect to the other particle and the environment. (b) Sketch of the diode in backward direction. (c) Normalized mean spectral in-plane Poynting vector and its amplitude $\left(\mathrm{Jm}^{-2}\right.$, colorbar) for the $m=+1$ particle resonance for the diode in forward direction. (d) as in (c) but for the backward case and for the $m=-1$ particle resonance. See also (Ott and Biehs, 2020).

modes (Bliokh and Nori, 2012) shows a spin momentum locking (Mechelen and Jacob, 2016), meaning that the waves with $k_{x}>0$ and $k_{x}<0$ have a different spin direction, the splitting can again be understood as a Zeeman splitting (Khandekar and Jacob, 2019a; Mechelen and Jacob, 2016).

Now, considering the situation in Fig. 44(a) and (b) the thermally excited localized modes of the hot nanoparticle can directly couple to the localized modes of the cold nanoparticle leading to a direct heat transfer between the particles. The thermally-excited localized modes of the hot particle can couple to the surface modes of the substrate, travel along the interface of the substrate and then couple to the localized modes of the cold nanoparticle so that in this case the heat is transferred between the two nanoparticles via the surface modes. Due to the non-reciprocity of the substrate the heat flow $\mathcal{P}_{2}$ in the forward direction in Fig. 44(a) and the heat flow $\mathcal{P}_{1}$ in the backward direction in Fig. 44(b) will be different, leading to a rectification effect $(\overline{\mathrm{Ott}}$ et al. $2019 \mathrm{a})$. A detailed analysis shows (Ott and Biehs, 2020) that there is a spin-selective coupling so that the localized modes couple preferably to the surface modes with the spin in the same direction. For example, the $m=-1(m=+1)$ resonance couples preferably to the surface modes with $k_{x}>0\left(k_{x}<0\right)$ providing the main heat flux channel in forward (backward) direction as shown in Fig. 44(c) (Fig. 44(d)). This can be also understood by a matching of the circularity of the particle resonances and the directionality of the interface resonances. The resulting rectification coefficient

$$
\eta=\frac{\mathcal{P}_{1}-\mathcal{P}_{2}}{\mathcal{P}_{1}}
$$

shown in Fig. 45 can be rather high even for relatively 


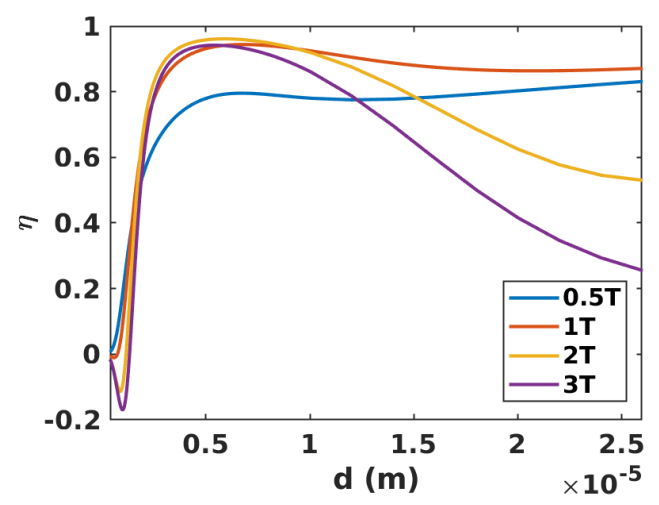

FIG. 45 Rectification coefficient from Eq. 136 for two InSb nanoparticles with $100 \mathrm{~nm}$ radius in $500 \mathrm{~nm}$ distance above an InSb substrate as sketched in Fig. 44 as a function of the interparticle distance $d$ for different magnetic field amplitudes. See also (Ott and Biehs, 2020, Ott et al. 2019a).

small magnetic fields. It should be kept in mind that when bringing the nanoparticles close to a substrate most of the heat will go to the substrate rather than to the other nanoparticle. Nonetheless, the rectification effect can result in a measurable heating of the cold nanoparticle (Ott and Biehs, 2020).

\section{OUTLOOK AND OPEN QUESTIONS}

While the heat transport mechanisms mediated by thermal photons in 1D and 3D systems have been intensively studied during the last decade they remain today unknown in $2 \mathrm{D}$ systems. Can we observe a diverging radiative conductivity with respect to system size as has already been predicted for the phononic conductivity in $2 \mathrm{D}$ anharmonic lattices? To answer this question and also identify different heat transport regimes in these systems, the scaling laws of radiative thermal conductance must be analyzed. Another fundamental problem is the crossover from $1 \mathrm{D}$ to $2 \mathrm{D}$ and from $3 \mathrm{D}$ to $2 \mathrm{D}$ systems. The spatial confinement of evanescent photons in these systems should play a key role in those transitions.

So far, dense many-body systems and effects like weak and strong localization for thermal radiation remain largely unexplored. In these strongly correlated systems, heat is typically carried through multiple connected channels associated with different heat carriers like electrons, phonons, and photons, which raises the question: under which conditions can one or more of these heat carries dominate heat transport? As hilighted in the introduction of this review, progress in unifying various transport mechanisms is beginning to be made, yet a complete theory capable of describing multichannel heat exchange in large many-body systems remains a challenge for understanding possible transport effects associated with coupling across such different channels.

As the number of bodies in interaction becomes large, the general formalism described in this review becomes numerically prohibitive. This is a serious issue to investigate heat transport in many-body systems in presence of long range interactions. A continuous description of heat transport in these systems could make the study of these systems feasible and it could in the same time be a powerful tool to study the NFRHT in mesoscopic physics or to make calculations of NFRHT between objects of arbitrary shape. Using the Chapman-Kolmogorov equation for the local temperature field, a Fokker-Planck equation can be derived and written in the hydrodynamic limit as an advection-diffusion equation which depends on directly measurable macroscopic quantities like the effective diffusion coefficient and which could be easily solved with standard numerical methods.

When it comes to recent exploration of the spin and angular momentum of thermally fluctuating fields, nearly all investigations have focused on single particles or semiinfinite materials. However, a corresponding general Nbody theory should be straight forwardly derived using the general framework presented in this review. This extension could pave the way to studies of thermal-field spin and angular momentum transport in atomic and molecular systems. Since magneto-optical effects based on the use of magneto-optical materials or Weyl semi-metals reported thus far have been relatively small, further studies aimed at enhancing these effects should be considered in the future, for instance by exploiting ferromagnetic or more strongly magnetic materials.

Non-Hermitian physics has attracted tremendeous interest during the last decade from a variety of fields in classical physics due to their mathematical equivalence with the Schrodinger equation, thus allowing one to mimic non-Hermitian wave physics with classical systems. Bipartite plasmonic and phonon-polaritonic manybody systems provide a natural platform to investigate such physics. Among their many peculiarities, one might point to the existence of original topological states that give rise to Berry-like phases and which may lead to the development of new materials such as topological insulators. These states and their consequences for the thermal management (active control of heat flux, heat pumping, heat flux focusing) remain largely unexplored in manybody systems.

Out-of equilibrium thermodynamics of many-body systems and its connections with information theory is also a future field of investigation. In systems with long-range interactions, the classical thermodynamic theory fails to describe the evolution of state variables since they cannot be sequenced in small independent parts. Normally, to calculate thermodynamic properties it is necessary to determine the microscopic states of a given system. However a phenomenological approach analogous to Landau's transition theory may be employed to study the thermodynamic behavior of these systems by considering macroscopic quantities. Hence, mechanisms such as phase transitions in magneto-optical systems could be investigated by analyzing the dependence of quantities like the ther- 
mal conductance or the entropy flux with order parameters such as the magnitude or orientation of a magnetic field.

The peculiarities of heat transfer in many-body systems has given rise to numerous development in the emerging field of thermotronics to manipulate heat flux in analogy with electric currents in electric circuits. This radical change of paradigm opens the way to a new generation of devices for active thermal management, innovative wireless sensors using heat as their primary source of energy, and to âĂIJlow-electricityâĂ ble of information processing. In these devices, infrared emission coming from various systems (people, machines, electric devicesâĂę) may for instance be captured by active thermal components to launch a sequence of logical operations in order to either control the heat propagation (modulate, amplify, split), trigger specific actions (opto-thermo-mechanical coupling with MEMS, thermal energy storageâĂę) or even process information. Hence the development of thermal logical circuits such as neural networks could open the door to a low-power and even zero-power communication technology for the Internet of Things, allowing machine-to-machine communication with heat. The design of thermal metamaterials such as thermal insulators, topological insulators or superdiffusive solids is also a promising challenge.

Finally, building experimental platforms based on multi-tip SThM setups, suspended membranes or even networks of electromechanical systems interacting at the nanometre-scale is one of the most important challenges for the next few years to measure the NFRHT in manybody systems, prove all already predicted effects and develop operational devices. In order to be able to have an access to conductance variations of few $\mathrm{nWK}^{-1}$, highsensitive heat flux sensors must be developed. This will require fabrication of thermometers working at the nanoscale and able to measure temperatures with an accuracy $<10 \mathrm{mK}$.

\section{Acknowledgments}

We thank all our colleagues within the nanoscale heat transfer community that engaged us in many fruitful interactions and spirited discussions. S.-A. B. acknowledges support from Heisenberg Programme of the Deutsche Forschungsgemeinschaft (DFG, German Research Foundation) under the project No. 404073166. J.C.C. acknowledges funding from the Spanish Ministry of Economy and Competitiveness (MINECO) (Contract No. FIS2017-84057-P). P. B.-A. Acknowledges support from Natural Sciences and Engineering Research Council of Canada through the RGPIN-2017 program-No.05445 and from the Agence Nationale de la Recherche in France through the ComputHeat project ANR-19-MRS1-0009.

\section{References}

Agarwal, G. S., 1975a, Phys. Rev. A 11, 230.

Agarwal, G. S., 1975b, Phys. Rev. A 11, 253.

Albaladejo, S., R. Gómez-Medina, L. S. Froufe-Pérez, H. Marinchio, R. Carminati, J. F. Torrado, G. Armelles, A. García-Martín, and J. J. Sáenz, 2010, Opt. Expr. 18, 3556.

Asheichyk, K., and M. Krüger, 2018, Phys. Rev. B 98, 195401.

Asheichyk, K., B. Müller, and M. Krüger, 2017, Phys. Rev. B 96, 155402.

Baibich, M. N., J. M. Broto, A. Fert, F. N. V. Dau, F. Petroff, P. Etienne, G. Creuzet, A. Friederich, and J. Chazelas, 1988, Phys. Rev. Lett. 61, 2472.

Barton, G., 2016, J. Stat. Phys. 165, 1153.

Basu, S., Y.-B. Chen, and Z. M. Zhang, 2007, Int. J. En. Res. 31, 689.

Basu, S., and M. Francoeur, 2011, Appl. Phys. Lett. 98, 243120 .

Basu, S., and S. Z. M. Zhang, 2009, Appl. Phys. Lett. 95, 133104.

Basu, S., Z. M. Zhang, and C. J. Fu, 2009, Int. J. En. Res. 33, 1203.

Becerril, D., and C. Noguez, 2019, Phys. Rev. B 99, 045418.

Ben-Abdallah, P., 2006, Appl. Phys. Lett. 89, 113117.

Ben-Abdallah, P., 2016, Phys. Rev. Lett. 116, 084301.

Ben-Abdallah, P., 2019a, Phys. Rev. B 99, 201406.

Ben-Abdallah, P., 2019b, Phys. Rev. Lett. 123, 264301.

Ben-Abdallah, P., A. Belarouci, L. Frechette, and S.-A. Biehs, 2015, Appl. Phys. Lett. 107, 053109.

Ben-Abdallah, P., and S.-A. Biehs, 2013, Appl. Phys. Lett. 103, 191907.

Ben-Abdallah, P., and S.-A. Biehs, 2014, Phys. Rev. Lett. 112, 044301.

Ben-Abdallah, P., and S.-A. Biehs, 2015, AIP Advances 5, 053502.

Ben-Abdallah, P., and S.-A. Biehs, 2016, Phys. Rev. B 94, 241401(R).

Ben-Abdallah, P., and S.-A. Biehs, 2017, Z. Naturforschung A 72, 151.

Ben-Abdallah, P., and S.-A. Biehs, 2019, Z. Naturforsch. A $\mathbf{7 4}, 689$.

Ben-Abdallah, P., S.-A. Biehs, and K. Joulain, 2011, Phys. Rev. Lett. 107, 114301.

Ben-Abdallah, P., and K. Joulain, 2010, Phys. Rev. B 82, $121419(\mathrm{R})$.

Ben-Abdallah, P., K. Joulain, J. Drevillon, and G. Domingues, 2009a, J.Appl. Phys. 106, 044306.

Ben-Abdallah, P., K. Joulain, J. Drevillon, and G. Domingues, 2009b, Appl. Phys. Lett. 94, 153117.

Ben-Abdallah, P., K. Joulain, J. Drevillon, and C. L. Goff, 2008, Phys. Rev. B 77, 075417.

Ben-Abdallah, P., K. Joulain, and A. Pryamikov, 2010, Appl. Phys. Lett. 96, 143117.

Ben-Abdallah, P., R. Messina, S.-A. Biehs, M. Tschikin, K. Joulain, and C. Henkel, 2013, Phys. Rev. Lett. 111, 174301.

Bergman, T. L., F. P. Incropera, D. P. DeWitt, and A. S. Lavine, 2011, Fundamentals of Heat and Mass Transfer (John Wiley \& Sons).

Bernardi, M. P., D. Milovich, and M. Francoeur, 2016, Nat. Commun. 7, 1.

Biehs, S.-A., 2007, Euro. Phys. J. B 58, 423. 
Biehs, S.-A., and G. S. Agarwal, 2013a, J. Opt. Soc. Am. B 30, 700 .

Biehs, S.-A., and G. S. Agarwal, 2013b, Appl. Phys. Lett. 103, 243112.

Biehs, S.-A., and P. Ben-Abdallah, 2016, Phys. Rev. B 93, 165405.

Biehs, S.-A., and P. Ben-Abdallah, 2017, Z. Naturforschung A 72, 115.

Biehs, S.-A., P. Ben-Abdallah, F. S. S. Rosa, K. Joulain, and J.-J. Greffet, 2011, Opt. Express 19, A1088.

Biehs, S.-A., and J.-J. Greffet, 2010a, Phys. Rev. Lett. 105, 234301.

Biehs, S.-A., and J.-J. Greffet, 2010b, Phys. Rev. B 82, 245410.

Biehs, S.-A., and J.-J. Greffet, 2010c, Phys. Rev. B 81, 245414.

Biehs, S.-A., O. Huth, and F. Rüting, 2008, Phys. Rev. B 78, 085414.

Biehs, S.-A., S. Lang, A. Y. Petrov, M. Eich, and P. BenAbdallah, 2015, Phys. Rev. Lett. 115, 174301.

Biehs, S.-A., V. M. Menon, and G. S. Agarwal, 2016, Phys. Rev. B 93, 245439.

Biehs, S.-A., D. Reddig, and M. Holthaus, 2007, Euro. Phys. J. B 55, 237.

Biehs, S.-A., O. H. F. Rüting, and M. Holthaus, 2010, J. Appl. Phys. 108, 014312.

Biehs, S.-A., M. Tschikin, and P. Ben-Abdallah, 2012, Phys. Rev. Lett. 109, 104301.

Biehs, S.-A., M. Tschikin, R. Messina, and P. Ben-Abdallah, 2013, Appl. Phys. Lett. 102, 131106.

Bimonte, G., 2009, Phys. Rev. A 80, 042102.

Bimonte, G., T. Emig, M. Kardar, and M. Krüger, 2017, Annual Review of Condensed Matter Physics 8, 119.

Bliokh, K., and F. Nori, 2012, Phys. Rev. A 85, 061801(R).

Bliokh, K. Y., and F. Nori, 2015, Phys. Rep. 592, 1.

Bouchet, D., D. Cao, R. Carminati, Y. D. Wilde, and V. Krachmalnicoff, 2016, Phys. Rev. Lett. 116, 037401.

Caloz, C., A. Alù, S. Tretyakov, D. Sounas, K. Achouri, and Z.-L. Deck-Léger, 2018, Phys. Rev. Applied 10, 0470011.

Castanié, E., R. Vincent, R. Pierrat, and R. Carminati, 2012, Int. J. Opt. 2012, 452047.

Chapuis, P.-O., M. Laroche, S. Volz, and J.-J. Greffet, 2008a, Appl. Phys. Lett. 92, 201906.

Chapuis, P.-O., M. Laroche, S. Volz, and J.-J. Greffet, 2008b, Phys. Rev. B 77, 125402.

Chapuis, P.-O., S. Volz, C. Henkel, K. Joulain, and J.-J. Greffet, 2008c, Phys. Rev. B 77, 035431.

Chen, K., P. Santhanam, and S. Fan, 2016, Phys. Rev. Appl. 6, 024014.

Chen, K., P. Santhanam, S. Sandhu, L. Zhu, and S. Fan, 2015, Phys. Rev. B 91, 134301.

Chen, Y., and Y. Xuan, 2015, Journal of Quantitative Spectroscopy and Radiative Transfer 158, 52 .

Chiloyan, V., J. Garg, K. Esfarjani, and G. Chen, 2015, Nat. Commun. 6, 1.

Chiu, K. W., and J. J. Quinn, 1972, Nuov. Cimento B 10, 1.

Choubdar, O. R., and M. Nikbakht, 2016, J. Appl. Phys. 120, 144303.

Cuevas, J. C., and F. J. García-Vidal, 2018, ACS Photonics $\mathbf{5}, 3896$.

Cuevas, J. C., and E. Scheer, 2017, Molecular Electronics: An Introduction to Theory and Experindidroth, R. M. A., A. García-Martín, and J. C. Cuevas, 2017, (World Scientific).

Cui, L., S. Hur, Z. A. Akbar, J. C. Klöckner, W. Jeong,
F. Pauly, S.-Y. Jang, P. Reddy, and E. Meyhofer, 2019, Nature 572, 628.

Cui, L., W. Jeong, V. Fernández-Hurtado, J. Feist, F. J. García-Vidal, J. C. Cuevas, E. Meyhofer, and P. Reddy, 2017a, Nat. Commun. 8, 14479.

Cui, L., W. Jeong, S. Hur, M. Matt, J. C. Klöckner, F. Pauly, P. Nielaba, J. C. Cuevas, E. Meyhofer, and P. Reddy, 2017b, Science 355, 1192.

Czapla, B., and A. Narayanaswamy, 2019, Journal of Quantitative Spectroscopy and Radiative Transfer 227, 4.

Dai, J., S. A. Dyakov, S. I. Bozhevolnyi, and M. Yan, 2016a, Phys. Rev. B 94, 125431.

Dai, J., S. A. Dyakov, and M. Yan, 2015, Phys. Rev. B 92, 035419.

Dai, J., S. A. Dyakov, and M. Yan, 2016b, Phys. Rev. B 93, 155403.

Danz, N., R. WaldhÃd'usl, A. BrÃd'uer, and R. Kowarschik, 2002, J. Opt. Soc. Am. B 19, 412.

Datta, S., 1997, Electronic Transport in Mesoscopic Systems (Cambridge University Press).

Dedkov, G. V., and A. A. Kyasov, 2007, Tech. Phys. Lett. 33, 305.

Dedkov, G. V., and A. A. Kyasov, 2011, Eur. Phys. Lett. 93, 34001.

Deshmukh, R., S.-A. Biehs, E. Khwaja, T. Galfsky, G. S. Agarwal, and V. M. Menon, 2018, ACS Photonics 5, 2737.

DeSutter, J., L. Tang, and M. Francoeur, 2019, Nat. Nanotechnol. 14, 751.

Domingues, G., S. Volz, K. Joulain, and J.-J. Greffet, 2005, Phys. Rev. Lett. 94, 085901.

Domoto, G., R. F. Boehm, and C. L. Tien, 1970, J. Heat Trans. 92, 412 .

Dong, J., J. Zhao, and L. Liu, 2017a, Phys. Rev. B 95, 125411.

Dong, J., J. Zhao, and L. Liu, 2017b, Journal of Quantitative Spectroscopy and Radiative Transfer 197, 114.

Dong, J., J. Zhao, and L. Liu, 2018, Phys. Rev. B 97, 075422.

Dorofeyev, I., H. Fuchs, and J. Jersch, 2002, Phys. Rev. E 65, 026610.

Dorofeyev, I. A., 1998, Journal of Physics D: Applied Physics $\mathbf{3 1}, 600$.

Dorofeyev, I. A., and E. A. Vinogradov, 2011, Phys. Rep. 504, 75 .

Draine, B. T., 1988, Astrophys. J. 333, 848.

Dyakov, S. A., J. Dai, M. Yan, and M. Qiu, 2015a, Appl. Phys. Lett. 106, 064103.

Dyakov, S. A., J. Dai, M. Yan, and M. Qiu, 2015b, J. Phys. D: Appl. Phys. 48, 305104.

Dyakov, S. A., V. A. Semenenko, N. A. Gippius, and S. G. Tikhodeev, 2018, Phys. Rev. B 98, 235416.

Eckhardt, W., 1982, Z. Phys. B: Cond. Matt. 46, 85.

Eckhardt, W., 1984, Phys. Rev. A 29, 1991.

Edalatpour, S., J. DeSutter, and M. Francoeur, 2016, JQSRT 178, 14.

Edalatpour, S., and M. Francoeur, 2014, Journal of Quantitative Spectroscopy and Radiative Transfer 133, 364.

Edalatpour, S., and M. Francoeur, 2016, Phys. Rev. B 94, 045406.

Edalatpour, S., M. Čuma, T. Trueax, R. Backman, and M. Francoeur, 2015, Phys. Rev. E 91, 063307.

Ekeroth, R. M. A., P. Ben-Abdallah, J. C. Cuevas, and A. García-Martín, 2018, ACS Photonics 5, 705. Phys. Rev. B 95, 235428.

Fan, L., Y. Guo, G. T. Papadakis, B. Zhao, Z. Zhao, S. Bud- 
dhiraju, M. Orenstein, and S. Fan, 2020, Phys. Rev. B 101, 085407.

Fernández-Hurtado, S., F. J. García-Vidal, S. Fan, and J. C. Cuevas, 2017, Phys. Rev. Lett. 118, 203901.

Ferreiros, Y., A. A. Zyuzin, and J. H. Bardarson, 2017, Phys. Rev. B 96, 115202.

Fiorino, A., D. Thompson, L. Zhu, R. Mittapally, S.-A. Biehs, O. Bezencenet, N. El-Bondry, S. Bansropun, P. BenAbdallah, E. Meyhofer, and P. Reddy, 2018a, ACS Nano 12, 5774 .

Fiorino, A., D. Thompson, L. Zhu, B. Song, P. Reddy, and E. Meyhofer, 2018b, Nano Lett. 18, 3711.

Fiorino, A., L. Zhu, D. Thompson, R. Mittapally, P. Reddy, and E. Meyhofer, 2018c, Nat. Nanotechn. 13, 806.

Francoeur, M., M. P. Mengüç, and R. Vaillon, 2008, Appl. Phys. Lett. 93, 043109.

Francoeur, M., M. P. Menguc, and R. Vaillon, 2009, Journal of Quantitative Spectroscopy and Radiative Transfer 110, 2002 .

Francoeur, M., M. P. Mengüç, and R. Vaillon, 2010a, J. Phys. D: Applied Physics 43, 075501.

Francoeur, M., M. P. Mengüc, and R. Vaillon, 2011, Phys. Rev. B 84, 075436.

Francoeur, M., M. P. Mengü $\tilde{A} \breve{g}$, and R. Vaillon, 2010b, J. Appl. Phys. 107, 034313.

Fujimoto, S., 2009, Phys. Rev. Lett 103, 047203.

Ghashami, M., H. Geng, T. Kim, N. Iacopino, S. K. Cho, and K. Park, 2018, Phys. Review Lett. 120, 175901.

Golyk, V. A., M. Krüger, and M. Kardar, 2013, Phys. Rev. B 88, 155117.

Gu, W., G.-H. Tang, and W.-Q. Tao, 2015, Int. J. Heat and Mass Transf. 82, 429.

Gu, Y., and K. G. Kornev, 2010, J. Opt. Soc. Am. B 27, 2165.

Guérout, R., J. Lussange, F. S. S. Rosa, J.-P. Hugonin, S. A. R. Dalvit, J.-J. Greffet, A. Lambrecht, and S. Reynaud, 2012, Phys. Rev. B 85, 180301.

Guha, B., C. Otey, C. B. Poitras, S. Fan, and M. Lipson, 2012, Nano Lett. 12, 4546.

Guo, Y., G. L. Cortes, S. Molesky, and Z. Jacob, 2012, Appl. Phys. Lett. 101, 131106.

Guo, Y., and Z. Jacob, 2013, Opt. Express 21, 15014.

Guo, Y., and Z. Jacob, 2014, J. Appl. Phys. 115, 234306.

Hall, E. H., 1879, Am. J. Math. 2, 287.

Hargreaves, C. M., 1969, Phys. Lett. A 30, 491.

Harrington, R. F., 1989, J. Electromagnet. Wav. 3, 1.

He, M.-J., H. Qi, Y. Li, Y.-T. Ren, W.-H. Cai, and L.-M. Ruan, 2019a, Int. J. Heat and Mass Transf. 137, 12.

He, M.-J., H. Qi, Y.-T. Ren, Y.-J. Zhao, and M. Antezza, 2019b, Appl. Phys. Lett. 115, 263101.

He, M.-J., H. Qi, Y.-F. Wang, Y.-T. Ren, W.-H. Cai, and L.-M. Ruan, 2019c, Opt. Expr. 27, A954.

Herz, F., and S.-A. Biehs, 2019, Eur. Phys. Lett. 127, 44001.

Howell, J. R., M. P. Menguc, and R. Siegel, 2016, Thermal Radiation Heat Transfer (CRC press).

Hu, L., A. Narayanaswamy, X. Chen, and G. Chen, 2008, Appl. Phys. Lett. 92, 133106.

Huang, Z.-M., B. Han, and M. Stone, 2020, Phys. Rev. B 101, 125201.

Hugonin, J.-P., M. Besbes, and P. Ben-Abdallah, 2015, Phys. Rev. B 91, 180202.

Huth, O., F. Rüting, S.-A. Biehs, and M. Holthaus, 2010, EPJ Appl. Phys. 50, 10603.

Iizuka, H., and S. Fan, 2018, Phys. Rev. Lett. 120, 063901.

Ilic, O., M. Jablan, J. D. Joannopoulos, I. Celanovic, H. Bul- jan, and M. SoljaÄøï̈̆G, 2012a, Phys. Rev. B 85, 155422. Ilic, O., M. Jablan, J. D. Joannopoulos, I. Celanovic, and M. Soljačić, 2012b, Opt. Express 20, A366.

Imry, Y., and R. Landauer, 1999, Rev. of Mod. Phys. 71, S306.

Incardone, R., T. Emig, and M. Krüger, 2014, EPL 106, 41001.

Inyushkin, A. V., and A. N. Taldenkov, 2007, JETP Lett. 86, 379.

Ito, K., A. Miura, H. Iizuka, and H. Toshiyoshi, 2015, Appl. Phys. Lett. 106, 083504.

Ito, K., K. Nishikawa, H. Iizuka, and H. Toshiyoshi, 2014, Appl. Phys. Lett. 105, 253503.

Ito, K., K. Nishikawa, A. Miura, H. Toshiyoshi, and H. Iizuka, 2017, Nano Lett. 17, 4347.

Janowicz, M., D. Reddig, and M. Holthaus, 2003, Phys. Rev. A 68, 043823.

Jin, W., R. Messina, and A. W. Rodriguez, 2017a, Opt. Express 25, 14746.

Jin, W., R. Messina, and A. W. Rodriguez, 2017b, Phys. Rev. B 95, 161409.

Jin, W., S. Molesky, Z. Lin, and A. W. Rodriguez, 2019, Phys. Rev. B 99, 041403.

Jin, W., A. G. Polimeridis, and A. W. Rodriguez, 2016, Phys. Rev. B 93, 121403.

Joulain, K., R. Carminati, J.-P. Mulet, and J.-J. Greffet, 2003, Phys. Rev. B 68, 245405.

Joulain, K., J. Drevillon, and P. Ben-Abdallah, 2010, Phys. Rev. B 81, 165119.

Joulain, K., Y. Ezzahri, J. Drevillon, and P. Ben-Abdallah, 2015, Appl. Phys. Lett. 106, 133505.

Joulain, K., J.-P. Mulet, F. Marquier, R. Carminati, and J.-J. Greffet, 2005, Surf. Sci. Rep. 57, 59.

Kan, Y. H., C. Y. Zhao, and Z. M. Zhang, 2019, Phys. Rev. B 99, 035433.

Karplus, R., and J. M. Luttinger, 1954, Phys. Rev. 95, 1154.

Kathmann, C., R. Messina, P. Ben-Abdallah, and S.-A. Biehs, 2018, Phys. Rev. B 98, 115434.

Kathmann, C., M. Reina, R. Messina, P. Ben-Abdallah, and S.-A. Biehs, 2020, Sci. Rep. 10, 3596.

Katsura, H., N. Nagaosa, and P. A. Lee, 2010, Phys. Rev. Lett 104, 066403.

Kemp, J. C., 1970, Astrophys. J. 162, 169.

Kemp, J. C., J. B. Swedlund, J. D. Landstreet, and J. R. P. Angel, 1970, Astrophys. J. 161, L77.

Khandekar, C., and Z. Jacob, 2019a, New J. Phys. 21, 103030.

Khandekar, C., and Z. Jacob, 2019b, Phys. Rev. Applied 12, 014053.

Khandekar, C., and A. W. Rodriguez, 2017, Appl. Phys. Lett. 111, 083104.

Kim, K., B. Song, V. Fernández-Hurtado, W. Lee, W. Jeong, L. Cui, D. Thompson, J. Feist, M. T. H. Reid, F. J. GarcíaVidal, J. C. Cuevas, E. Meyhofer, et al., 2015, Nature 528, 387.

Kittel, A., W. Müller-Hirsch, J. Parisi, S.-A. Biehs, D. Reddig, and M. Holthaus, 2005, Phys. Rev. Lett. 95, 224301.

Kittel, A., U. W. J. Welker, O. Huth, F. Rüting, and S.-A. Biehs, 2008, Appl. Phys. Lett. 93, 193109.

Klimchitskaya, G. L., and V. M. Mostepanenko, 2015, Phys. Rev. B 91, 045412.

Kloppstech, K., N. Könne, S.-A. Biehs, A. W. Rodriguez, L. Worbes, D. Hellmann, and A. Kittel, 2017, Nat. Commun. 8, 14475.

Kollyukh, O. G., A. I. Liptuga, V. Morozhenko, and V. I. 
Pipa, 2005, Phys. Rev. B 71, 073306.

Komiyama, S., 2019, Journal of Applied Physics 125, 010901.

Králík, T., P. Hanzelka, M. Zobac, V. Musilová, T. Fořt, and M. Horak, 2012, Phys. Rev. Lett. 109, 224302.

Králík, T., V. Musilová, T. Fořt, and A. Srnka, 2017, Phys. Rev. B 95, 060503(R).

Krüger, M., G. Bimonte, T. Emig, and M. Kardar, 2012, Phys. Rev. B 86, 115423.

Krüger, M., T. Emig, and M. Kardar, 2011, Phys. Rev. Lett. 106, 210404.

Krüger, M., V. A. Golyk, G. Bimonte, and M. Kardar, 2013, EPL 104, 41001.

Kubo, R., 1966, Rep. Progr. Phys. 29, 255.

Kubytskyi, V., S.-A. Biehs, and P. Ben-Abdallah, 2014, Phys. Rev. Lett. 113, 074301.

Lakhtakia, A., 1992, International Journal of Modern Physics C 3, 583.

Lakhtakia, A., V. K. Varadan, and V. V. Varadan, 1991, Int. J. Infrarared and Millimeter Waves 12, 1253.

Lang, S., G. Sharma, S. Molesky, P. U. KrÃd'nzien, T. Jalas, Z. Jacob, A. Y. Petrov, and M. Eich, 2017, Sci. Rep. 7, 13916.

Lang, S., M. Tschikin, S.-A. Biehs, A. Y. Petrov, and M. Eich, 2015, Appl. Phys. Lett. 104, 121903.

Langlais, M., J.-P. Hugonin, M. Besbes, and P. Ben-Abdallah, 2014, Opt. Express 22, A577.

Laroche, M., R. Carminati, and J.-J. Greffet, 2006, J. Appl. Phys. 100, 063704.

Latella, I., and P. Ben-Abdallah, 2017, Phys. Rev. Lett. 118, 173902.

Latella, I., P. Ben-Abdallah, S.-A. Biehs, M. Antezza, and R. Messina, 2017, Phys. Rev. B 95, 205404.

Latella, I., S.-A. Biehs, R. Messina, A. W. Rodriguez, and P. Ben-Abdallah, 2018a, Phys. Rev. B 97, 035423.

Latella, I., O. Marconot, J. Sylvestre, L. G. Frechette, and P. Ben-Abdallah, 2019, Phys. Rev. Appl. 11, 024004.

Latella, I., R. Messina, S.-A. Biehs, J. M. Rubi, and P. BenAbdallah, 2020, Sci. Rep. 10, 8938.

Latella, I., R. Messina, J. M. Rubi, and P. Ben-Abdallah, 2018b, Phys. Rev. Lett. 121, 023903.

Latella, I., A. Pérez-Madrid, J. M. Rubi, S.-A. Biehs, and P. Ben-Abdallah, 2015, Phys. Rev. Applied 4, 011001.

Latella, I., A. Prez-Madrid, L. Lapas, and J. Rubi, 2014, J. Appl. Phys. 115, 124307.

Lau, W. T., J.-T. Shen, and S. Fan, 2009, Phys. Rev. B 80, 155135.

Lau, W. T., J.-T. Shen, G. . Veronis, S. Fan, and P. V. Braun, 2008, Appl. Phys. Lett. 92, 103106.

Leduc, M. A., 1887, J. Phys. 2e SÃl'rie 6, 378.

Lévy, P , 1937, Théorie De lâAZAddition Des Var (Gauthier-Villars, Paris).

Li, B., L. Wang, and G. Casati, 2006, Appl. Phys. Lett. 88, 143501.

Lim, M., S. S. Lee, and B. J. Lee, 2013, Opt. Express 21, 22173.

Lim, M., S. S. Lee, and B. J. Lee, 2015, Phys. Rev. B 91, 195136.

Liu, J., and E. Narimanov, 2015, Phys. Rev. B 91, 041403(R).

Liu, X., J. Shen, and Y. Xuan, 2019, Nanoscale and Microscale Thermophysical Engineering 23, 188.

Liu, X., R. Z. Zhang, and Z. Zhang, 2014a, ACS Photonics 1, 785.

Liu, X., and Z. M. Zhang, 2015a, ACS Photonics 2, 1320.

Liu, X., B. Zhao, and Z. M. Zhang, 2015, Phys. Rev. A 91,
062510

Liu, X. L., R. Z. Zhang, and Z. M. Zhang, 2013, Appl. Phys. Lett. 103, 213102.

Liu, X. L., R. Z. Zhang, and Z. M. Zhang, 2014b, International Journal of Heat and Mass Transfer 73, 389.

Liu, X. L., and Z. M. Zhang, 2015b, Appl. Phys. Lett. 107, 143114

Luo, C., A. Narayanaswamy, G. Chen, and J. D. Joannopoulos, 2004, Phys. Rev. Lett. 93, 213905.

Luo, M., J. Dong, J. Zhao, L. Liu, and M. Antezza, 2019, Phys. Rev. B 99, 134207.

Manjavacas, A., and F. J. G. de Abajo, 2012, Phys. Rev. B 86, 075466.

Martynenko, Y. V., and L. I. Ognev, 2005, Techn. Phys. 50, 1522.

Maslovski, S. I., C. R. Simovski, and S. A. Tretyakov, 2013, Phys. Rev. B 87, 155124.

McCauley, A. P., M. T. H. Reid, M. Krüger, and S. G. Johnson, 2012, Phys. Rev. B 85, 165104.

Mechelen, T. V., and Z. Jacob, 2016, Optica 3, 118.

Menges, F., M. Dittberner, L. Novotny, D. Passarello, S. S. P. Parkin, M. Spieser, H. Riel, and B. Gotsmann, 2016, Appl. Phys. Lett. 108, 171904.

Messina, R., and M. Antezza, 2011a, Europhys. Lett. 95, 61002.

Messina, R., and M. Antezza, 2011b, Phys. Rev. A 84, 042102

Messina, R., and M. Antezza, 2014, Phys. Rev. A 89, 052104.

Messina, R., M. Antezza, and P. Ben-Abdallah, 2012, Phys. Rev. Lett. 109, 244302.

Messina, R., and P. Ben-Abdallah, 2013, Sci. Rep. 3, 1383.

Messina, R., and P. Ben-Abdallah, 2020, Phys. Rev. B 101, 165435 .

Messina, R., P. Ben-Abdallah, B. Guizal, and M. Antezza, 2017a, Phys. Rev. B 96, 045402.

Messina, R., P. Ben-Abdallah, B. Guizal, M. Antezza, and S.-A. Biehs, 2016a, Phys. Rev. B 94, 104301.

Messina, R., S.-A. Biehs, and P. Ben-Abdallah, 2018, Phys. Rev. B 97, 165437.

Messina, R., J.-P. Hugonin, J. Greffet, F. Marquier, Y. D. Wilde, A. Belarouci, L. Frechette, Y. Cordier, and P. BenAbdallah, 2013a, Phys. Rev. B 87, 085421.

Messina, R., W. Jin, and A. W. Rodriguez, 2016b, Phys. Rev. B 94, 121410

Messina, R., A. Noto, B. Guizal, and M. Antezza, 2017b, Phys. Rev. B 95, 125404.

Messina, R., M. Tschikin, S.-A. Biehs, and P. Ben-Abdallah, 2013b, Phys. Rev. B 88, 104307.

Miller, D. A. B., 2000, Appl. Opt. 39, 1681.

sMiller, D. A. B., 2007, J. Opt. Soc. Am. B 24, A1.

Miller, O. D., S. G. Johnson, and A. W. Rodriguez, 2014, Phys. Rev. Lett. 112, 157402.

Miller, O. D., S. G. Johnson, and A. W. Rodriguez, 2015, Phys. Rev. Lett. 115, 204302.

Miller, O. D., A. G. Polimeridis, M. T. H. Reid, C. W. Hsu, B. G. DeLacy, J. D. Joannopoulos, M. Soljačić, and S. G. Johnson, 2016, Opt. Express 24, 3329.

Modest, M. F., 2013, Radiative Heat Transfer (Academic press).

Molesky, S., P. S. Venkataram, W. Jin, and A. W. Rodriguez, 2020, Phys. Rev. B 101, 035408.

Moncada-Villa, E., and J. C. Cuevas, 2020, Phys. Rev. B 101, 085411.

Moncada-Villa, E., V. Fernández-Hurtado, F. J. García- 
Vidal, A. García-Martín, and J. C. Cuevas, 2015, Phys. Rev. B 92, 125418.

Mosso, N., U. Drechsler, F. Menges, P. Nirmalraj, S. Karg, H. Riel, and B. Gotsmann, 2017, Nat. Nanotechnol. 12, 430.

Mulet, J.-P., K. Joulain, R. Carminati, and J.-J. Greffet, 2001, Appl. Phys. Lett. 78, 2931.

Mulet, J.-P., K. Joulain, R. Carminati, and J.-J. Greffet, 2002, Microscale Thermophys. Eng. 6, 209.

Müller, B., R. Incardone, M. Antezza, T. Emig, and M. Krüger, 2017, Phys. Rev. B 95, 085413.

Musilová, V., T. Králík, T. Fořt, and M. Macek, 2019, Phys. Rev. B 99, 024511.

Nagaosa, N., J. Sinova, S. Onoda, A. H. MacDonald, and N. P. Ong, 2010, Rev. Mod. Phys. 82, 1539.

Narayanaswamy, A., and G. Chen, 2003, Appl. Phys. Lett. $\mathbf{8 2}, 3544$.

Narayanaswamy, A., and G. Chen, 2005, JQSRT 93, 175.

Narayanaswamy, A., and G. Chen, 2008, Phys. Rev. B 77, 075125 .

Narayanaswamy, A., S. Shen, and G. Chen, 2008, Phys. Rev. B 78, 115303.

Nefedov, I. S., and C. R. Simovski, 2011, Phys. Rev. B 84, 195459.

Neto, A. H. C., F. Guinea, N. M. R. Peres, K. S. Novoselov, and A. K. Geim, 2009, Rev. Mod. Phys. 81, 109.

Newman, W. D., C. L. Cortes, A. Afshar, K. Cadien, A. Meldrum, R. Fedosejevs, and Z. Jacob, 2018, Science Advances 4, eaar5278.

Nikbakht, M., 2014, J. Appl. Phys. 116, 094307.

Nikbakht, M., 2015, EPL 110, 14004.

Nikbakht, M., 2017, Phys. Rev. B 96, 125436.

Novotny, L., and B. Hecht, 2006, Principles of Nano-Optics (Cambridge University Press).

Onose, Y., T. Ideue, H. Katsura, Y. Shiomi, N. Nagaosa, and Y. Tokura, 2010, Science 329, 297.

Onsager, L., 1931, Phys. Rev. 37, 405.

Ordonez-Miranda, J., L. Tranchant, S. Gluchko, and S. Volz, 2015, Phys. Rev. B 92, 115409.

Ordonez-Miranda, J., L. Tranchant, K. Joulain, Y. Ezzahri, J. Drevillon, and S. Volz, 2016, Phys. Rev. B 93, 035428.

Otey, C., and S. Fan, 2011a, Phys. Rev. B 84, 245431.

Otey, C., and S. Fan, 2011b, Phys. Rev. B 84, 245431.

Otey, C. R., W. T. Lau, and S. Fan, 2010, Phys. Rev. Lett. 104, 154301.

Otey, C. R., L. Zhu, S. Sandhu, and S. Fan, 2014, JQSRT $132,3$.

Ott, A., P. Ben-Abdallah, and S.-A. Biehs, 2018, Phys. Rev. B 97, 205414.

Ott, A., and S.-A. Biehs, 2020, Phys. Rev. 101, 155428.

Ott, A., S.-A. Biehs, and P. Ben-Abdallah, 2020, Phys. Rev. B 101, 241411(R).

Ott, A., R. Messina, P. Ben-Abdallah, and S.-A. Biehs, 2019a, J. Photon. Energy 9, 032711.

Ott, A., R. Messina, P. Ben-Abdallah, and S.-A. Biehs, 2019b, Appl. Phys. Lett. 114, 163105.

Ottens, R. S., V. Quetschke, S. Wise, A. A. Alemi, R. Lundock, G. Mueller, D. H. Reitze, D. B. Tanner, and B. F. Whiting, 2011, Phys. Rev. Lett. 107, 014301.

Papadakis, G. T., B. Zhao, S. Buddhiraju, and S. Fan, 2019, ACS Photonics 6, 709.

Pendry, J. B., 1999, J. Phys.: Cond. Matter 11, 6621.

Pendry, J. B., 2000, Phys. Rev. Lett. 85, 3966.

Pendry, J. B., L. Martin-Moreno, and F. J. Garcia-Vidal,
2004, Science 305, 847.

Pérez-Madrid, A., J. M. Rubi, and L. C. Lapas, 2008, Phys. Rev. B 77, 155417.

Pineider, F., G. Campo, V. Bonanni, C. de Julian Fernandez, G. Mattei, A. Caneschi, D. Gatteschi, and C. Sangregorio, 2013, Nano Lett. 13, 4785.

Planck, M., 1914, Blakiston's Son \& Co .

Poddubny, A., I. Iorsh, P. Belov, and Y. Kivshar, 2013, Nat. Photonics 7, 948.

Polder, D., and M. V. Hove, 1971, Phys. Rev. B 4, 3303.

Polimeridis, A. G., M. T. H. Reid, W. Jin, S. G. Johnson, J. K. White, and A. W. Rodriguez, 2015, Phys. Rev. B 92, 134202.

Polimeridis, A. G., M. T. H. Reid, S. G. Johnson, J. K. White, and A. W. Rodriguez, 2015, IEEE Transactions on Antennas and Propagation 63, 611.

Poudel, A., X. Chen, and M. A. Ratner, 2016, J. Phys. Chem. Lett. 7, 955.

ProdâÁŹhomme, H., J. Ordonez-Miranda, Y. Ezzahri, J. Drévillon, and K. Joulain, 2016, J. Appl. Phys. 119, 194502.

ProdâĂŹhomme, H., J. Ordonez-Miranda, Y. Ezzahri, J. Drévillon, and K. Joulain, 2018, Journal of Quantitative Spectroscopy and Radiative Transfer 210, 52 .

Purcell, E. M., and R. C. Pennypacker, 1973, Astrophys. J. 186, 707 .

Raj, B., M. V. de Voorde, and Y. Mahajan, 1995, Nanotechnology for Energy Sustainability (Wiley).

Ramirez, F. V., and A. J. H. McGaughey, 2017, Phys. Rev. B 96, 165428 .

Reid, M. T. H., and S. G. Johnson, 2015, IEEE Transactions on Antennas and Propagation 63, 3588.

Reid, M. T. H., O. D. Miller, A. G. Polimeridis, A. W. Rodriguez, E. M. Tomlinson, and S. G. Johnson, 2017, arXiv preprint arXiv:1708.01985 .

Reid, M. T. H., A. W. Rodriguez, and S. G. Johnson, 2013a, Proceedings of the IEEE 101, 531.

Reid, M. T. H., J. White, and S. G. Johnson, 2013b, Phys. Rev. A 88, 022514.

Rengarajan, S. R., and Y. Rahmat-Samii, 2000, IEEE Antennas and Propagation Magazine 42, 122.

Righi, A., 1887, Mem. Acc. Lincei 4, 433.

Rodriguez, A. W., O. Ilic, P. Bermel, I. Celanovic, J. D. Joannopoulos, M. SoljaÄı̈̈̈̆G, and S. G. Johnson, 2011, Phys. Rev. Lett. 107, 114302.

Rodriguez, A. W., M. T. H. Reid, and S. G. Johnson, 2013a, Phys. Rev. B 88, 054305.

Rodriguez, A. W., M. T. H. Reid, J. Varela, J. D. Joannopoulos, F. Capasso, and S. G. Johnson, 2013b, Phys. Rev. Lett. 110, 014301.

Rodriguez-Lopez, P., W.-K. Tse, and D. A. R. Dalvit, 2015, J. Phys.: Condens. Matter 27, 214019.

Rousseau, E., A. Siria, G. Jourdan, S. Volz, F. Comin, J. Chevrier, and J.-J. Greffet, 2009, Nat. Photonics 3, 514.

Rüting, F., S.-A. Biehs, O. Huth, and M. Holthaus, 2012, Phys. Rev. B 82, 115443.

Rytov, S. M., Y. A. Kravtsov, and V. I. Tatarskii, 1989, Priniciples of Statistical Radiophysics. 3. (Springer).

Sääskilahti, K., J. Oksanen, and J. Tulkki, 2014, Phys. Rev. B 89, 134301.

Salihoglu, H., V. Iyer, T. Taniguchi, K. Watanabe, P. D. Ye, and X. Xu, 2019, Adv. Funct. Mater. , 1905830.

Sasihithlu, K., 2019, J. Phot. Energie 9, 032709.

Sasihithlu, K., and G. S. Agarwal, 2018, Nanophotonics 7, 
1581.

Sasihithlu, K., and A. Narayanaswamy, 2011, Phys. Rev. B, 83, 161406(R).

Sernelius, B. E., 2012, Phys. Rev. B 85, 195427.

Shen, S., A. Mavrokefalos, P. Sambegoro, and G. Chen, 2012, Appl. Phys. Lett. 100, 233114.

Shen, S., A. Narayanaswamy, and G. Chen, 2009, Nano Lett. 9, 2909 .

Shi, J., P. Li, B. Liu, and S. Shen, 2013, Appl. Phys. Lett. 102, 183114.

Shi, K., F. Bao, and S. He, 2017, ACS Photonics 4, 971.

Shi, K., R. Liao, G. Cao, F. Bao, and S. He, 2018, Opt. Express 26, A591.

Shi, K., Y. Sun, Z. Chen, N. He, F. Bao, J. Evans, and S. He, 2019a, Nano Lett. 19, 8082.

Shi, K. Z., F. L. Bao, N. He, and S. L. He, 2019b, International Journal of Heat and Mass Transfer 134, 1119.

Shlesinger, M. F., G. M. Zaslavsky, and U. Frisch, 1995, Lévy Flights and Related Topics in Physicss (SpringerVerlag, Berlin).

Silveirinha, M. G., 2017, Phys. Rev. B 95, 115103.

Simchi, H., 2017, J. Appl. Phys. 121, 094301.

Simovski, C., S. Maslovski, I. Nefedov, and S. Tretyakov, 2013, Opt. Express 21, 14988.

Song, B., A. Fiorino, E. Meyhofer, and P. Reddy, 2015a, AIP advances 5, 053503.

Song, B., Y. Ganjeh, S. Sadat, D. Thompson, A. Fiorino, V. Fernández-Hurtado, J. Feist, F. J. Garcia-Vidal, J. C. Cuevas, P. Reddy, and E. Meyhofer, 2015b, Nat. Nanotechnol. 10, 253.

Song, B., D. Thompson, A. Fiorino, Y. Ganjeh, P. Reddy, and E. Meyhofer, 2016, Nat. Nanotechnol. 11, 509.

Song, J., Q. Cheng, Z. Luo, X. Zhou, and Z. Zhang, 2019, Int. J. Heat and Mass Transf. 140, 80.

Song, J. L., L. Lu, Q. Cheng, and Z. X. Luo, 2018, J. Heat Transfer 140, 082005.

St-Gelais, R., B. Guha, L. Zhu, S. Fan, and M. Lipson, 2014, Nano Letters 14, 6971.

St-Gelais, R., L. Zhu, S. Fan, and M. Lipson, 2016, Nat. Nanotechnol. 11, 15.

Strohm, C., G. L. J. A. Rikken, and P. Wyder, 2005, Phys. Rev. Lett. 95, 155901.

Svetovoy, V. B., and G. Palasantzas, 2014, Phys. Rev. Applied 2, 034006 .

Svetovoy, V. B., P. J. van Zwol, and J. Chevrier, 2012, Phys. Rev. B 85, 155418.

Tervo, E., M. Francoeur, B. A. Cola, and Z. M. Zhang, 2019a, Phys. Rev. B 100, 205422.

Tervo, E. J., O. S. Adewuyi, J. S. Hammonds, and B. A. Cola, 2016, Materials Horizons 3, 434.

Tervo, E. J., B. A. Cola, and Z. M. Zhang, 2020, JQSRT 246, 106947.

Tervo, E. J., M. E. Gustafson, Z. M. Zhang, B. A. Cola, and M. A. Filler, 2019b, Appl. Phys. Lett. 114, 163104.

Thomas, N. H., M. C. Sherrott, J. Broulliet, H. A. Atwater, and A. J. Minnich, 2019, Nano Lett. 19, 3898.

Thompson, D., L. Zhu, E. Meyhofer, and P. Reddy, 2020, Nat. Nanotechnol. 15, 99.

Tomchuk, P. M., and N. I. Grigorchuk, 2006, Phys. Rev. B 73, 155423.

Tretyakov, S., 2014, Plasmonics 9, 935.

Tschikin, M., P. Ben-Abdallah, and S.-A. Biehs, 2012a, Phys. Lett. A 376, 3462.

Tschikin, M., S.-A. Biehs, P. Ben-Abdallah, S. Lang, A. Y.
Petrov, and M. Eich, 2015, JQSRT 17, 158.

Tschikin, M., S.-A. Biehs, R. Messina, and P. Ben-Abdallah, 2013, Journal of Optics 15, 105101.

Tschikin, M., S.-A.Biehs, P. Ben-Abdallah, and F. S. S. Rosa, 2012b, EPJ B 85, 233.

Velizhanin, K. A., and T. V. Shahbazyan, 2012, Phys. Rev. B 86, 245432.

Venkataram, P. S., J. Hermann, A. Tkatchenko, and A. W. Rodriguez, 2018, Phys. Rev. Lett. 121, 045901.

Venkataram, P. S., S. Molesky, W. Jin, and A. W. Rodriguez, 2020, Phys. Rev. Lett. 124, 013904.

Volokitin, A. I., and B. N. J. Persson, 2001, Phys. Rev. B 63, 205404.

Volokitin, A. I., and B. N. J. Persson, 2002, Phys. Rev. B 65, 115419.

Volokitin, A. I., and B. N. J. Persson, 2004, Phys. Rev. B 69, 045417.

Volokitin, A. I., and B. N. J. Persson, 2007, Rev. Mod. Phys. $\mathbf{7 9}, 1291$.

Volokitin, A. I., and B. N. J. Persson, 2011, Phys. Rev. B 83, 241407.

Wang, J.-S., and J. Peng, 2017, EPL 118, 24001.

Wang, Y., and J. Wu, 2016, AIP Advances 6, 025104.

Weber, W. H., and G. W. Ford, 2004, Phys. Rev. B 70, 125429.

Weick, G., and D. Weinmann, 2011, Phys. Rev. B 83, 125405.

Wen, S.-B., 2010, Journal of Heat Transfer 132.

Werner, G. R., C. A. Bauer, and J. R. Cary, 2013, Journal of Computational Physics 255, 436.

Worbes, L., D. Hellmann, and A. Kittel, 2013, Phys. Rev. Lett. 110, 134302.

Wunsch, B., T. Stauber, F. Sols, and F. Guinea, 2006, New Journal of Physics 8, 318.

Xiao, S., X. Zhu, B.-H. Li, and N. A. Mortensen, 2016, Frontiers of Physics 11, 117801.

Yang, J., W. Du, Y. Su, Y. Fu, S. Gong, S. He, and Y. Ma, 2018, Nat. Commun. 9, 1.

Yang, Y., S. Basu, and L. Wang, 2013, Appl. Phys. Lett. 103, 163101.

Yannopapas, V., and N. V. Vitanov, 2013, Phys. Rev. Lett. 110, 044302.

Zhang, Y., M. Antezza, H.-L. Yi, and H.-P. Tan, 2019a, Phys. Rev. B 100, 085426.

Zhang, Y., H.-L. Yi, and H.-P. Tan, 2018, ACS Photonics 5, 3739.

Zhang, Y., H.-L. Yi, H.-P. Tan, and M. Antezza, 2019b, Phys. Rev. B 100, 134305.

Zhang, Z. M., 2007, Nano/Microscale Heat Transfer (McGraw-Hill).

Zhao, B., K. Chen, S. Buddhiraju, G. Bhatt, M. Lipson, and S. Fan, 2017a, Nano Energy 41, 344.

Zhao, B., B. Guizal, Z. M. Zhang, S. Fan, and M. Antezza, 2017b, Phys. Rev. B 95, 245437.

Zheng, Z., and Y. Xuan, 2011, Nanoscale and Microscale Thermophys. Eng. 15, 237.

Zhu, L., and S. Fan, 2014, Phys. Rev. B 90, 220301(R).

Zhu, L., and S. Fan, 2016, Phys. Rev. Lett. 117, 134303.

Zhu, L., Y. Guo, and S. Fan, 2018, Phys. Rev. B 97, 094302.

Zundel, L., and A. Manjavacas, 2020, Phys. Rev. Applied 13, 054054 .

van Zwol, P., L. Ranno, and J. Chevrier, 2012a, Phys. Rev. Lett. 108, 234301.

van Zwol, P., S. Thiele, C. Berger, W. A. de Heer, and J. Chevrier, 2012b, Phys. Rev. Lett. 109, 264301. 
van Zwol, P. J., K. Joulain, P. B. Abdallah, J.-J. Greffet, and J. Chevrier, 2011a, Phys. Rev. B 83, 201404.

van Zwol, P. J., K. Joulain, P. Ben-Abdallah, and J. Chevrier, 2011b, Phys. Rev. B 84, 161413. 